\title{
QUANTUM SUPERGROUPS IV. THE MODIFIED FORM
}

\author{
SEAN CLARK
}

\begin{abstract}
We construct a canonical basis for a class of tensor product modules of a quantum covering group associated to an Kac-Moody Lie superalgebra, and use these bases to construct a canonical basis for the modified form of a quantum covering group.
\end{abstract}

\section{Contents}

1. Introduction

2. The quantum covering groups

3. The modified quantum covering groups

4. Canonical bases

5. Inner product on $\dot{\mathbf{U}}$

6. Twistors

References

\section{INTRODUCTION}

1.1. The modified form of the Drinfeld-Jimbo quantum group $\mathbf{U}$ is a non-unital algebra $\dot{\mathbf{U}}$ which was first defined in type $A$ by Beilinson-Lusztig-MacPherson [BLM], and generalized to other types by Lusztig [L92]. The algebra $\mathbf{U}$ can be thought of as a version of $\mathbf{U}$ optimized for the study of weight $\mathbf{U}$ modules, which are also $\mathbf{U}$-modules. The modified form admits interesting higher-order structure that cannot be constructed on the whole quantum group. In particular, the Lusztig-Kashiwara canonical basis of the half-quantum group $\mathbf{U}^{-}$L90, K91] can be extended to a canonical basis of $\mathbf{U}$ [L92, K93, and moreover U admits a categorification KL, Web13.

Together with Hill and Wang CW, CHW1, CHW2, we defined and systematically developed the theory of covering quantum groups associated to an anisotropic Kac-Moody superalgebra. These algebras, whose definition is inspired by the results in [CW, [HW], combine the structure and representation theories of a classical quantum group and a quantum supergroup by using a "half parameter" $\pi$, satisfying $\pi^{2}=1$, in the place of super signs; one recovers the classical quantum group under the specialization $\pi=1$, and the quantum supergroup under the specialization $\pi=-1$. Moreover, the half-quantum covering group and the integrable modules admit (compatible) canonical bases which both generalize the Lusztig-Kashiwara canonical bases and specialize to canonical bases for the corresponding superalgebra upon setting $\pi=-1$.

The modified form of the rank one quantum covering group appeared in [CW], and there it is shown that this modified form retains many of the nice characteristics of its non-covering counterpart. In particular, it has a canonical basis which is structurally similar to the canonical basis of quantum $\mathfrak{s l}_{2}$, as well as a bilinear form, with respect to which the Chevalley generators are essentially adjoint. A definition of the modified form in higher rank can be found in [CFLW]. 
Like their non-covering counterparts, quantum covering groups have strong ties to results in categorification. Indeed, the quiver Hecke superalgebras introduced by Kang, Kashiwara, and Tsuchioka [KKT] (also see [W, EKL]) provide an alternate categorification of the half-quantum group. They also provide a categorification of certain types of half-quantum supergroups as shown by Hill and Wang [HW]. These alternate categorifications glue together to a categorification of the half-quantum covering group. Moreover, Kang, Kashiwara, and Oh KKO have shown that the cyclotomic quiver Hecke superalgebras categorify the integrable modules (for rank one, also see [EKL, HW]). The modified form of the rank one quantum covering group was recently shown to admit a (super)categorification by Ellis and Lauda [EL].

1.2. The goal of this paper is to provide an intrinsic construction of a canonical basis for the modified form of a quantum covering group, as well as develop auxiliary structure, such as a bilinear form, using parallel techniques to those utilized in Lusztig's book [L93, Part IV].

Using the structure and representation theory of quantum covering groups, we deduce a number of structural properties for the modified form which parallel the structure theory of modified quantum groups. Following Lusztig's construction of a bar-involution on tensor products, we use (a variant of) the quasi- $\mathcal{R}$-matrix defined in [CHW1] to exhibit a bar-involution on a family of modules $\mathcal{F}$. This bar-involution can be used to construct a canonical basis for the modules which can be "glued together" to yield a canonical basis $\dot{\mathcal{B}}$ for the modified form. This canonical basis generalizes the canonical basis of Lusztig for the modified quantum groups, in the sense that $\left.\dot{\mathcal{B}}\right|_{\pi=1}$ is precisely Lusztig's canonical basis. On the other hand, $\left.\dot{\mathcal{B}}\right|_{\pi=-1}$ furnishes a canonical basis for the modified form of a Kac-Moody quantum supergroup of anisotropic type.

A surprising connection between the modified forms of non-super and super quantum groups is that they can be realized as rational forms of a complex algebra. This identification can be succintly realized as an automorphism of the complexification of the modified form of the quantum covering group. This map and the maps it induces on both the quantum covering group and the half-quantum covering group are called twistors. These maps were defined and studied in [CFLW] (extending work in rank one by Fan and Li FL).

The twistor is remarkably useful for deducing properties of quantum covering groups from the corresponding properties of quantum groups. However, the definition of the twistor relies on a choice of total order on the simple roots, and its highly non-canonical nature is not suitable for deducing the existence of a canonical basis of the quantum covering group. Nonetheless, it happens that the twistor matches the canonical basis of $\mathbf{U}^{-}$with itself up to a factor of an integral power of $\sqrt{-1}$; see [CFLW, Theorem 3.6]. We will build on the results in loc. cit. to extend the notion of twistor to (complexifications of) the modules in $\mathcal{F}$. These module twistors preserve the canonical bases (up to an integral power of $\sqrt{-1}$ ) as well. As a consequence, we show that the canonical basis $\dot{\mathcal{B}}$ of $\dot{\mathbf{U}}$ is also preserved (up to scalar multiple) by the twistor.

We will also define a bilinear form on the modified quantum covering group which generalizes the bilinear form on the half-quantum covering group. This bilinear form is shown to exhibit a number of nice qualities; namely, it is invariant under some of the important automorphisms of the quantum covering group, and it can be viewed as a limit of bilinear forms on the tensor product modules. We note that in rank one, the bilinear form we shall define differs from the one defined in rank one in $\mathrm{CW}$, but they are intimately related; see Proposition 5.8. The bilinear form in loc. cit. is inadequate from the point of view of categorification (see Remark 5.9), but we will show this deficiency 
can be explained by utilizing an additional symmetry of the quantum covering group to relate these forms.

Given the Ellis-Lauda categorification in rank one [EL and the construction of odd knot invariants associated to finite type quantum covering groups by Mikhaylov and Witten [MW], we expect that quantum covering groups and their modified form will be an important setting for obtaining further categorifications and categorical odd knot invariants. We believe that the bilinear form in this paper will be useful for constructing categorifications of modified quantum covering groups of higher rank. However, we note that, in rank one, our bilinear form differs from the one proposed by Ellis and Lauda, which appears to be a skew-version of the bilinear form defined in Proposition 5.8. Nonetheless, the canonical bases we construct agree with the earlier construction in [CW], which appear as indecomposable 1-morphisms in the Ellis-Lauda categorification.

We also note that there exist canonical bases of Lie superalgebras outside of anisotropic Kac-Moody type. In [CHW3], canonical bases are constructed for $\mathfrak{g l}(n \mid 1)$ and $\mathfrak{o s p}(2 \mid 2 n)$ by a quantum shuffle approach. This technique tells us very little about the relation of the canonical basis and the representation theory, but it is expected that for polynomial modules, the canonical basis will descend to a basis of the module (cf. [CHW3, §8]). If this is true, then the canonical bases of loc. cit. should have an extension to a suitable modified form (say, with idempotents corresponding to polynomial weights). There is some evidence that this should be possible; for example, see El Turkey and Kujawa's presentation of Schur superalgebras [ETK].

1.3. The paper is structured in the following way. In Section 2, we will recall the definitions and basic results about the quantum covering groups. We will also prove some new technical results we will need in later sections.

In Section 3, we define the modified quantum group $\dot{\mathbf{U}}$ and study its structure in detail. In particular, we show that $\dot{\mathbf{U}}$ is approximated by certain tensor product modules in a way analogous to the approximation of $\mathbf{U}^{-}$by simple highest weight modules.

In Section 4, we construct canonical bases for the family of modules $\mathcal{F}$. The main result of this section is the construction of a canonical basis of $\dot{U}$ which is simultaneously compatible with the canonical bases of the $\mathcal{F}$. This basis is a generalization of the canonical basis of $\mathbf{U}^{-}$which was constructed in [CHW2].

In Section 5, we present a construction of a bilinear form on $\dot{\mathbf{U}}$ with nice properties. We further show that this bilinear form can be viewed as a limit of suitable bilinear forms on modules, which has the consequence of showing that the canonical basis of $\dot{\mathbf{U}}$ is $\pi$-almost-orthonormal.

Finally, in Section 6 we discuss some extensions of the results in [CFLW]. In particular, we show that any module in $\mathcal{F}$ admits a twistor map. We then show that twistors preserve the canonical bases of $\dot{\mathbf{U}}$ and of modules in $\mathcal{F}$ up to a power of $\sqrt{-1}$.

Acknowledgements: I am indebted to Weiqiang Wang, as this paper would be nonexistent without his guidance. I would like to thank David Hill, Yiqiang Li, and Zhaobing Fan for the fruitful collaborations on related projects. I thank Aaron Lauda for many helpful comments and stimulating conversations; in particular, for pointing out that the bilinear form in [CW] has a defect with respect to categorification.

\section{The QUANTUM COVERING GROUPS}

In this section, we will recall the essential definitions and results from earlier papers. We caution the reader that while the results are largely restatements of results in CHW1, 
CHW2, several of our conventions differ from those in loc. cit. We will also present some new results which will be of use later on; specifically, Lemmas 2.7, 2.12, and 2.14.

\subsection{The Cartan datum.}

Definition 2.1. A Cartan datum is a pair $(I, \cdot)$ consisting of a finite set $I$ and a $\mathbb{Z}$-valued symmetric bilinear form $\nu, \nu^{\prime} \mapsto \nu \cdot \nu^{\prime}$ on the free abelian group $\mathbb{Z}[I]$ satisfying

(a) $d_{i}=\frac{i \cdot i}{2} \in \mathbb{Z}_{>0}, \quad \forall i \in I$

(b) $a_{i j}=2 \frac{i \cdot j}{i \cdot i} \in \mathbb{Z}_{\leq 0}$, for $i \neq j$ in $I$.

A Cartan datum is called an anisotropic super Cartan datum (or anisotropic datum, in brief) if there is a partition $I=I_{\overline{0}} \amalg I_{\overline{1}}$ which satisfies the anisotropic condition

(c) $2 \frac{i \cdot j}{i \cdot i} \in 2 \mathbb{Z}$ if $i \in I_{\overline{1}}$.

The $i \in I_{\overline{0}}$ are called even, $i \in I_{\overline{1}}$ are called odd. We define a parity function $p: I \rightarrow\{0,1\}$ so that $i \in I_{\overline{p(i)}}$. An anisotropic datum is called bar-consistent if it additionally satisfies

(d) $d_{i} \equiv p(i) \bmod 2, \quad \forall i \in I$.

We will always assume an anisotropic Cartan datum satisfies $I_{\overline{1}} \neq \emptyset$ and is barconsistent in this paper. We note that a bar-consistent anisotropic datum satisfies

$$
i \cdot j \in 2 \mathbb{Z} \quad \text { for all } i, j \in I \text {. }
$$

Let $\nu=\sum_{i \in I} \nu_{i} i \in \mathbb{Z}[I]$. We extend the parity function on $I$ to the $\mathbb{Z}$-module homomorphism $p: \mathbb{Z}[I] \rightarrow \mathbb{Z}_{2}$ by

$$
p(\nu)=\sum_{i \in I} \nu_{i} p(i)
$$

We define the height function ht on $\mathbb{Z}[I]$ by letting

$$
\operatorname{ht}(\nu)=\sum_{i \in I} \nu_{i}
$$

We define the notation

$$
\tilde{\nu}=\sum d_{i} \nu_{i} i
$$

A root datum associated to a anisotropic datum $(I, \cdot)$ consists of

(a) two finitely generated free abelian groups $Y, X$ and a perfect bilinear pairing $\langle\cdot, \cdot\rangle: Y \times X \rightarrow \mathbb{Z}$

(b) an embedding $I \subset X\left(i \mapsto i^{\prime}\right)$ and an embedding $I \subset Y(i \mapsto i)$ satisfying

(c) $\left\langle i, j^{\prime}\right\rangle=\frac{2 i \cdot j}{i \cdot i}$ for all $i, j \in I$.

If the image of the imbedding $I \subset X$ (respectively, the image of the imbedding $I \subset Y$ ) is linearly independent in $X$ (respectively, in $Y$ ), then we say that the root datum is $X$-regular (resp. $Y$-regular). In this paper, we will assume the root datum is always $Y$-regular.

Remark 2.2. For many results, the $Y$-regular condition can be relaxed using similar arguments to those in [L93, Part IV].

If $V$ is a vector space graded by $\mathbb{Z}[I], X$, or $Y$, we will use the weight notation $|x|=\mu$ if $x \in V_{\mu}$. If $V$ is a $\mathbb{Z}_{2}$-graded vector space, we will use the parity notation $p(x)=a$ if $x \in V_{a}$. 
2.2. Parameters. Let $v$ be a formal parameter and let $\pi$ be an indeterminate such that

$$
\pi^{2}=1 \text {. }
$$

For a commutative ring $R$ with 1 , we will form a new ring $R^{\pi}=R[\pi] /\left(\pi^{2}-1\right)$. Given an $R^{\pi}$-module (or algebra) $M$, the specialization of $M$ at $\pi= \pm 1$ means the $R$-module (or algebra) $\left.M\right|_{\pi= \pm 1}=R_{ \pm} \otimes_{R^{\pi}} M$, where $R_{ \pm}=R$ is viewed as a $R^{\pi}$-module on which $\pi$ acts as \pm 1 .

Assume 2 is invertible in $R$; i.e. $\frac{1}{2} \in R$. We define

$$
\varepsilon_{+}=\frac{1+\pi}{2}, \quad \varepsilon_{-}=\frac{1-\pi}{2},
$$

and note that $R^{\pi}=R \varepsilon_{+} \oplus R \varepsilon_{-}$. In particular, since $\pi \varepsilon_{ \pm}= \pm \varepsilon_{ \pm}$for an $R^{\pi}$-module $M$, we see that

$$
\left.M\right|_{\pi= \pm 1} \cong \varepsilon_{ \pm} M
$$

The principal rings of concern in this paper are $\mathbb{Q}(v)^{\pi}$ and $\mathbb{A}=\mathbb{Z}^{\pi}\left[v^{ \pm 1}\right]$. For $k \in \mathbb{Z}_{\geq 0}$ and $n \in \mathbb{Z}$, we use a $(v, \pi)$-variant of quantum integers, quantum factorial and quantum binomial coefficients:

$$
\begin{aligned}
& {[n]_{v, \pi} }=\frac{(\pi v)^{n}-v^{-n}}{\pi v-v^{-1}} \in \mathbb{A}, \\
& {[n]_{v, \pi}^{!} }=\prod_{l=1}^{n}[l]_{v, \pi} \in \mathbb{A}, \\
& {\left[\begin{array}{l}
n \\
k
\end{array}\right]_{v, \pi}=\frac{\prod_{l=n-k+1}^{n}\left((\pi v)^{l}-v^{-l}\right)}{\prod_{m=1}^{k}\left((\pi v)^{m}-v^{-m}\right)} \in \mathbb{A} . }
\end{aligned}
$$

We will use the notation

$$
v_{i}=v^{d_{i}}, \quad \pi_{i}=\pi^{d_{i}}, \quad \text { for } i \in I .
$$

More generally, for $\nu=\sum \nu_{i} i$, we set

$$
v_{\nu}=\prod_{i \in I} v_{i}^{\nu_{i}}, \quad \pi_{\nu}=\prod_{i \in I} \pi_{i}^{\nu_{i}} .
$$

There are two important automorphisms of $\mathbb{Q}(v)^{\pi}$ and $\mathbb{A}$ for our purposes. The bar involution on $\mathbb{Q}(v)^{\pi}$ is the $\mathbb{Q}^{\pi}$-algebra automorphism defined by $\overline{f(v, \pi)}=f\left(\pi v^{-1}, \pi\right)$ for $f(v, \pi) \in \mathbb{Q}(v)^{\pi}$. The dagger involution on $\mathbb{Q}(v)^{\pi}$ is the $\mathbb{Q}^{\pi}$-algebra automorphism defined by $f(v, \pi)^{\dagger}=f(\pi v, \pi)$ for $f(v, \pi) \in \mathbb{Q}(v)^{\pi}$. We note that both involutions restict to $\mathbb{Z}^{\pi}$-algebra automorphisms of $\mathbb{A}$.

Observe that the $(v, \pi)$-integers are bar-invariant, but are not $\dagger$-invariant in general; to wit,

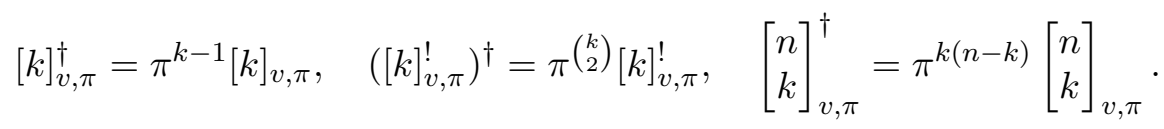

In particular, we note that $\left[\begin{array}{l}n \\ k\end{array}\right]_{v, \pi}$ is $\dagger$-invariant if and only if $n$ is odd or $k$ is even.

Remark 2.3. We note that $[n]^{\dagger}=\frac{v^{n}-(\pi v)^{-n}}{v-\pi v^{-1}}$ could well be the definition of $(v, \pi)$-integers, and we regard it as an alternate convention. 
2.3. The quantum covering groups. For the rest of the paper, we fix an anisotropic datum $(I, \cdot)$ and associated root datum $(Y, X,\langle\cdot, \cdot\rangle)$. We recall some definitions from CHW1.

Definition 2.4. CHW1 The half-quantum covering group $\mathbf{f}$ associated to the anisotropic datum $(I, \cdot)$ is the $\mathbb{Z}[I]$-graded $\mathbb{Q}(v)^{\pi}$-algebra on the generators $\theta_{i}$ for $i \in I$ with $\left|\theta_{i}\right|=i$, satisfying the relations

$$
\sum_{k=0}^{b_{i j}}(-1)^{k} \pi^{\left(\begin{array}{c}
k \\
2
\end{array}\right) p(i)+k p(i) p(j)}\left[\begin{array}{c}
b_{i j} \\
k
\end{array}\right]_{v_{i}, \pi_{i}} \theta_{i}^{b_{i j}-k} \theta_{j} \theta_{i}^{k}=0 \quad(i \neq j),
$$

We define the divided powers

$$
\theta_{i}^{(n)}=\theta_{i}^{n} /[n]_{v_{i}, \pi_{i}}^{!} .
$$

Let ${ }_{\mathbb{A}} \mathbf{f}$ be the $\mathbb{A}$-algebra generated by $\theta_{i}^{(n)}$ for various $i \in I, n \in \mathbb{N}$.

Definition 2.5. CHW1 The quantum covering group $\mathbf{U}$ associated to the root datum $((I, \cdot), Y, X,\langle\cdot, \cdot\rangle)$ is the $\mathbb{Q}(v)^{\pi}$-algebra with generators $E_{i}, F_{i}, K_{\mu}$, and $J_{\mu}$, for $i \in I$ and $\mu \in Y$, subject to the relations:

$$
\begin{gathered}
J_{\mu} J_{\nu}=J_{\mu+\nu}, \quad K_{\mu} K_{\nu}=K_{\mu+\nu}, \quad K_{0}=J_{0}=J_{\nu}^{2}=1, \quad J_{\mu} K_{\nu}=K_{\nu} J_{\mu}, \\
J_{\mu} E_{i}=\pi^{\left\langle\mu, i^{\prime}\right\rangle} E_{i} J_{\mu}, \quad J_{\mu} F_{i}=\pi^{-\left\langle\mu, i^{\prime}\right\rangle} F_{i} J_{\mu}, \\
K_{\mu} E_{i}=v^{\left\langle\mu, i^{\prime}\right\rangle} E_{i} K_{\mu}, \quad K_{\mu} F_{i}=v^{-\left\langle\mu, i^{\prime}\right\rangle} F_{i} K_{\mu}, \\
E_{i} F_{j}-\pi^{p(i) p(j)} F_{j} E_{i}=\delta_{i j} \frac{J_{d_{i} i} K_{d_{i} i}-K_{-d_{i} i}}{\pi_{i} v_{i}-v_{i}^{-1}}, \\
\sum_{k=0}^{b_{i j}}(-1)^{k} \pi^{\left(\begin{array}{c}
k \\
2
\end{array}\right) p(i)+k p(i) p(j)}\left[\begin{array}{c}
b_{i j} \\
k
\end{array}\right]_{v_{i}, \pi_{i}} E_{i}^{b_{i j}-k} E_{j} E_{i}^{k}=0 \quad(i \neq j), \\
\sum_{k=0}^{b_{i j}}(-1)^{k} \pi^{\left(\begin{array}{l}
k \\
2
\end{array}\right) p(i)+k p(i) p(j)}\left[\begin{array}{c}
b_{i j} \\
k
\end{array}\right]_{v_{i}, \pi_{i}} F_{i}^{b_{i j}-k} F_{j} F_{i}^{k}=0(i \neq j),
\end{gathered}
$$

for $i, j \in I$ and $\mu, \nu \in Y$.

The algebras $\mathbf{U}$ and $\mathbf{f}$ are related in the following way. Let $\mathbf{U}^{-}$be the subalgebra generated by $F_{i}$ with $i \in I, \mathbf{U}^{+}$be the subalgebra generated by $E_{i}$ with $i \in I$, and $\mathbf{U}^{0}$ be the subalgebra generated by $K_{\nu}$ and $J_{\nu}$ for $\nu \in Y$. There is an isomorphisms $\mathbf{f} \rightarrow \mathbf{U}^{-}$ (resp. $\mathbf{f} \rightarrow \mathbf{U}^{+}$) defined by $\theta_{i} \mapsto \theta_{i}^{-}=F_{i}$ (resp. $\theta_{i} \mapsto \theta_{i}^{+}=E_{i}$ ).

Proposition 2.6. There is a triangular decomposition

$$
\mathbf{U} \cong \mathbf{U}^{-} \otimes \mathbf{U}^{0} \otimes \mathbf{U}^{+} \cong \mathbf{U}^{+} \otimes \mathbf{U}^{0} \otimes \mathbf{U}^{-} .
$$

We define the divided powers

$$
E_{i}^{(n)}=\left(\theta_{i}^{(n)}\right)^{+}, \quad F_{i}^{(n)}=\left(\theta_{i}^{(n)}\right)^{-},
$$

and set ${ }_{\mathbb{A}} \mathbf{U}^{ \pm}=\left({ }_{\mathbb{A}} \mathbf{f}\right)^{ \pm}$. We will also use the shorthand notation

$$
\tilde{J}_{\nu}=J_{\tilde{\nu}}, \quad \tilde{K}_{\nu}=K_{\tilde{\nu}} .
$$

Then for $\nu \in Y$, we also have the $\nu$-integers and $\nu$-binomial coefficients

$$
[\nu ; n]=\frac{\pi_{\nu}^{n} v_{\nu}^{n} \tilde{J}_{\nu} \tilde{K}_{\nu}-\tilde{K}_{\nu}^{-1} v_{\nu}^{-n}}{\pi_{\nu} v_{\nu}-v_{\nu}^{-1}}, \quad\left[\begin{array}{c}
\nu ; n \\
k
\end{array}\right]=\frac{\prod_{s=1}^{k}[\nu ; n+1-k]}{[k]_{v_{\nu}, \pi_{\nu}}^{!}} .
$$


We let ${ }_{\mathbb{A}} \mathbf{U}$ be the $\mathbb{A}$-subalgebra of $\mathbf{U}$ generated by $E_{i}^{(n)}, F_{i}^{(n)}, J_{\nu}, K_{\nu}$, and $\left[\begin{array}{c}i ; n \\ k\end{array}\right]$ for $i \in I$, $\nu \in Y, n \geq a \in \mathbb{N}$.

The algebra $\mathbf{U}$ has a number of important gradings. We endow $\mathbf{U}$ with a $\mathbb{Z}[I]$-grading by setting

$$
\left|E_{i}\right|=i, \quad\left|F_{i}\right|=-i, \quad\left|J_{\mu}\right|=\left|K_{\mu}\right|=0,
$$

and also endow $\mathbf{U}$ with a $\mathbb{Z}_{2}$-grading by setting

$$
p\left(E_{i}\right)=p\left(F_{i}\right)=p(i), \quad p\left(J_{\mu}\right)=p\left(K_{\mu}\right)=0 .
$$

We set $\mathbf{U}_{\nu}=\{x \in \mathbf{U}:|x|=\nu\}$. Note that $p(x)=p(\nu)$ for all $x \in \mathbf{U}_{\nu}$.

The algebra $\mathbf{U}$ has a number of important automorphisms, which we will now recall. There is a $\mathbb{Q}(v)^{\pi}$-algebra automorphism $\omega: \mathbf{U} \rightarrow \mathbf{U}$ defined by

$$
\omega\left(E_{i}\right)=F_{i}, \quad \omega\left(F_{i}\right)=\pi_{i} \tilde{J}_{i} E_{i}, \quad \omega\left(K_{\nu}\right)=K_{-\nu}, \quad \omega\left(J_{\nu}\right)=J_{\nu} .
$$

(Note that $\omega$ is not an involution, and our $\omega$ corresponds to $\omega^{-1}$ in the notation of CHW1]). For any $\mathbf{U}$-module $M$, we let ${ }^{\omega} M$ be the $\omega$-twisted module; that is, the space $M$ equipped with a new action $\cdot$ defined by $u \cdot m=\omega(u) m$. We also extend this notation to powers of $\omega$.

There is also an important anti-automorphism of $\mathbf{U}$. To wit, there is a $\mathbb{Q}(v)^{\pi}$-linear map $\rho: \mathbf{U} \rightarrow \mathbf{U}$ such that

$$
\rho\left(E_{i}\right)=\pi_{i} \tilde{J}_{i} E_{i}, \quad \rho\left(F_{i}\right)=F_{i}, \quad \rho\left(K_{\nu}\right)=K_{-\nu}, \quad \rho\left(J_{\nu}\right)=J_{\nu},
$$

and satisfying

$$
\rho(x y)=\rho(y) \rho(x) .
$$

Finally, the bar-involution on $\mathbf{U}$ is the $\mathbb{Q}^{\pi}$-algebra automorphism defined by

$$
\bar{E}_{i}=E_{i}, \quad \bar{F}_{i}=F_{i}, \quad \bar{K}_{\nu}=J_{\nu} K_{-\nu}, \quad \bar{J}_{\nu}=J_{\nu}, \quad \bar{v}=\pi v^{-1} .
$$

The maps $\omega, \rho$, and - (or variations thereof) were defined in CHW1.

We also have a $\mathbb{Q}^{\pi}$-linear automorphism $\dagger$ on $\mathbf{U}$ extending $\dagger: \mathbb{Q}(v)^{\pi} \rightarrow \mathbb{Q}(v)^{\pi}$.

Lemma 2.7. There exists a $\mathbb{Q}^{\pi}$-algebra automorphism $\dagger: \mathbf{U} \rightarrow \mathbf{U}$, denoted by $\bullet \mapsto \bullet \dagger$, such that

$$
E_{i}^{\dagger}=\pi_{i} \tilde{J}_{i} E_{i}, \quad F_{i}^{\dagger}=F_{i}, \quad K_{\nu}^{\dagger}=J_{\nu} K_{\nu}, \quad J_{\nu}^{\dagger}=J_{\nu}, \quad v^{\dagger}=\pi v .
$$

Proof. To see that $\dagger$ is a well-defined map, we may check that the images of the generators satisfy the defining relations. All of the relations are trivial to verify except the Serre relations when $p(i) \neq 0$. However, since $b_{i j}=1-a_{i j}$ is odd in this case, the binomial coefficients are dagger-invariant. Then (2.11) is dagger-invariant, and the image of (2.10) is proportional to itself (by a factor of $\pi^{b_{i j} p(i)+p(j)} \tilde{J}_{b_{i j} i+j}$ ).

Remark 2.8. One interpretation of the automorphism $\dagger$ is as follows. There is an algebra $\mathbf{U}^{\dagger}$ with generators $E_{i}^{\dagger}, F_{i}^{\dagger}, J_{\nu}^{\dagger}, K_{\nu}^{\dagger}(i \in I, \nu \in Y$ ), and subject to (2.6)-(2.11) (with $E_{i}, F_{i}, J_{\nu}, K_{\nu}$ replaced by $E_{i}^{\dagger}, F_{i}^{\dagger}, J_{\nu}^{\dagger}, K_{\nu}^{\dagger}$ ) except with (2.9) replaced by

$$
E_{i}^{\dagger} F_{j}^{\dagger}-\pi^{p(i) p(j)} F_{j}^{\dagger} E_{i}^{\dagger}=\delta_{i j} \frac{\tilde{K}_{i}^{\dagger}-\tilde{J}_{i}^{\dagger} \tilde{K}_{-i}^{\dagger}}{v_{i}-\pi_{i} v_{i}^{-1}} .
$$

The algebra $\mathbf{U}^{\dagger}$ may be thought of as $\mathbf{U}$ defined with respect to the alternate convention of $(v, \pi)$-integers, and $\dagger$ defines a $\mathbb{Q}^{\pi}$-algebra isomorphism $\dagger: \mathbf{U} \rightarrow \mathbf{U}^{\dagger}$ defined by

$$
E_{i} \mapsto E_{i}^{\dagger}, \quad F_{i} \mapsto F_{i}^{\dagger}, \quad K_{\nu} \mapsto J_{\nu}^{\dagger} K_{\nu}^{\dagger}, \quad J_{\nu} \mapsto J_{\nu}^{\dagger}, \quad v \mapsto \pi v .
$$

This map will extend naturally to the modified form, but it is unclear what meaning $\dagger$ has in the categorification in EL]. 
2.4. Hopf structure. As in the case of classical quantized enveloping algebras, we may endow $\mathbf{U}$ with a Hopf covering algebra structure in several ways (cf. CHW1 for details on the counit and antipode). As different coproducts have been considered in the papers [CHW1, CHW2] (see Remark 2.10 below), we will describe some of the more natural choices for the coproducts. Their relationships are discussed further in Section 3.3 .

We have homomorphisms

$$
\Delta_{1}, \Delta_{2}, \Delta_{3}, \Delta_{4}: \mathbf{U} \rightarrow \mathbf{U} \otimes \mathbf{U}
$$

such that

$$
\begin{array}{rlrl}
\Delta_{1}\left(E_{i}\right) & =E_{i} \otimes 1+\tilde{J}_{i} \tilde{K}_{i} \otimes E_{i} & & \Delta_{1}\left(F_{i}\right)=F_{i} \otimes \tilde{K}_{-i}+1 \otimes F_{i} \\
\Delta_{2}\left(E_{i}\right)=E_{i} \otimes 1+\tilde{K}_{i} \otimes E_{i} & \Delta_{2}\left(F_{i}\right)=F_{i} \otimes \tilde{K}_{-i}+\tilde{J}_{i} \otimes F_{i} \\
\Delta_{3}\left(E_{i}\right) & =E_{i} \otimes \tilde{K}_{-i}+1 \otimes E_{i} & & \Delta_{3}\left(F_{i}\right)=F_{i} \otimes 1+\tilde{J}_{i} \tilde{K}_{i} \otimes F_{i} \\
\Delta_{4}\left(E_{i}\right) & =E_{i} \otimes \tilde{K}_{-i}+\tilde{J}_{i} \otimes E_{i} & \Delta_{4}\left(F_{i}\right)=F_{i} \otimes 1+\tilde{K}_{i} \otimes F_{i} \\
\Delta_{s}\left(K_{\mu}\right) & =K_{\mu} \otimes K_{\mu} \text { for } s=1,2,3,4 . & & \Delta_{s}\left(J_{\mu}\right)=J_{\mu} \otimes J_{\mu} \text { for } s=1,2,3,4 .
\end{array}
$$

It is elementary to verify that for each $s=1,2,3,4, \Delta_{s}\left({ }_{\mathbb{A}} \mathbf{U}\right) \subset{ }_{\mathbb{A}} \mathbf{U} \otimes_{\mathbb{A}} \mathbf{U}$. Given $\mathbf{U}$ modules $M$ and $M^{\prime}$, we denote by $M \otimes_{s} M^{\prime}$ the space $M \otimes_{\mathbb{Q}(q)^{\pi}} M^{\prime}$ with the $\mathbf{U}$-modules structure given by $\Delta_{s}$.

We recall from [CHW1, §3.1] the existence of a unique family of elements $\Theta_{1, \nu} \in$ $\mathbf{U}_{\nu}^{-} \otimes \mathbf{U}_{\nu}^{+}$such that the element $\Theta_{1}=\sum_{\nu \in \mathbb{N}[I]} \Theta_{1, \nu}$ satisfies

$$
\Delta_{1}(u) \Theta_{1}=\Theta_{1} \bar{\Delta}_{1}(u)
$$

for all $u \in \mathbf{U}$ (equality in a suitable completion of $\mathbf{U} \otimes \mathbf{U}$ ).

Proposition 2.9. There exists unique families of elements

$$
\begin{gathered}
\Theta_{2, \nu} \in \mathbf{U}_{\nu}^{-} \otimes\left(\tilde{J}_{\nu} \mathbf{U}_{\nu}^{+}\right) \\
\Theta_{3, \nu} \in \mathbf{U}_{\nu}^{+} \otimes \mathbf{U}_{\nu}^{-} \\
\Theta_{4, \nu} \in\left(\tilde{J}_{\nu} \mathbf{U}^{+}\right)_{\nu} \otimes \mathbf{U}_{\nu}^{-}
\end{gathered}
$$

for $\nu \in \mathbb{N}[I]$ such that the element $\Theta_{s}=\sum_{\nu \in \mathbb{N}[I]} \Theta_{s, \nu}$ for $s=2,3,4$ satisfies

$$
\Delta_{s}(u) \Theta_{s}=\Theta_{s} \bar{\Delta}_{s}(u)
$$

for all $u \in \mathbf{U}$ (with equality in a suitable completion of $\mathbf{U} \otimes \mathbf{U}$ ). Moreover, $\Theta_{s} \bar{\Theta}_{s}=1$.

Proof. It is easy to see that the argument in the proof of [CHW1, Theorem 3.1.1] extends to other coproducts after making suitable modifications. Alternatively, this will follow from Lemma 3.6 and the corresponding properties of $\Theta_{1}$.

In this note, the coproduct of principal concern will be $\Delta_{3}$, and we use the shortened notations $\Delta=\Delta_{3}$ and $\otimes=\otimes_{3}$, and $\Theta=\Theta_{3}$.

Remark 2.10. We note that $\Delta_{1}$ is the main coproduct considered in CHW1 and $\Delta_{4}$ is the main coproduct considered in CHW2. The coproduct $\Delta_{1}$ was introduced without specific motivation, while $\Delta_{4}$ provides the most natural Tensor Product Rule for crystal bases (cf. [CHW2, Theorem 4.12]). We will use $\Delta_{3}$ since it admits a more natural quasi$\mathcal{R}$-matrix and is better for the construction of canonical bases for the modified quantum group; compare Theorem 4.14 and Corollary 4.15 . 
2.5. Highest weight modules and canonical bases. Recall that a weight module for $\mathbf{U}$ is a $\mathbf{U}$-module $M$ satisfying

$$
M=\bigoplus M_{\lambda}, \quad M_{\lambda}=\left\{m \in M \mid J_{\mu} K_{\nu} m=\pi^{\langle\mu, \lambda\rangle} v^{\langle\nu, \lambda\rangle} m, \mu, \nu \in Y\right\} .
$$

We can define integrable and highest weight modules as usual, and set $\mathcal{O}$ be the category of weight modules which are locally $\mathbf{U}^{+}$-finite.; cf. [CHW1 for more details.

Recall that for $\lambda \in X^{+}$, the $\mathbf{U}$-module $M(\lambda)$ is defined to be the space $\mathbf{f}$ with the U-action satisfying

$$
E_{i} 1=0, \quad F_{i} x=\theta_{i} x, \quad J_{\nu} K_{\mu} x=\pi^{\left\langle\nu,|x|^{\prime}\right\rangle} v^{\left\langle\mu,|x|^{\prime}\right\rangle} x .
$$

We call $M(\lambda)$ the Verma module. There is a submodule $\mathcal{T}$ of $M(\lambda)$ generated by $\theta_{i}^{\langle i, \lambda\rangle+1}$ for all $i \in I$; we call the quotient $V(\lambda)=M(\lambda) / \mathcal{T}$ the irreducible module of highest weight $\lambda$, and denote the image of 1 in this quotient by $\eta_{\lambda}$.

Remark 2.11. In fact, $V(\lambda)$ is not irreducible as a $\mathbf{U}$-module, as it is the sum $\left.V(\lambda)\right|_{\pi=1} \oplus$ $V(\lambda)_{\pi=-1}$ of irreducible U-modules (cf. CHW1, $\left.\S 2.7\right]$ ). However, if we define a $\pi$-free weight module as a weight module where the weight spaces are free $\mathbb{Q}(v)^{\pi}$-modules, then $V(\lambda)$ is an irreducible module in the category of $\pi$-free weight modules.

In the following, we also will often consider the module ${ }^{\omega} V(\lambda)$, which is called the irreducible module of lowest weight $-\lambda$. We denote the image of 1 in ${ }^{\omega} V(\lambda)$ by $\xi_{-\lambda}$. Though $\omega$ is not an involution, we note that the twisting the action on $V(\lambda)$ by $\omega^{2}$ does not yield a new module. To wit, we have the following lemma.

Lemma 2.12. The $\mathbb{Q}(v)^{\pi}$-linear isomorphism $\omega^{2}: V(\lambda) \rightarrow \omega^{2} V(\lambda)$ given by

$$
\omega^{2}(x)=\pi_{\nu} \pi^{\langle\tilde{\nu}, \lambda\rangle} x, \quad x \in V(\lambda)_{\lambda-\nu^{\prime}}, \quad \nu \in \mathbb{N}[I],
$$

is a $\mathbf{U}$-module isomorphism.

Proof. For any homogeneous $u \in \mathbf{U}_{\mu}, \omega^{2}(u)=\pi_{\mu} \tilde{J}_{\mu} u$. Then for $x \in V(\lambda)_{\lambda-\nu^{\prime}}$ with $\nu \in \mathbb{N}[I]$, we have $\omega^{2}(u x)=\pi_{\mu+\nu} \pi^{\langle\tilde{\mu}+\tilde{\nu}, \lambda\rangle} u x=\omega^{2}(u) \omega^{2}(x)$.

Since $M(\lambda)=\mathbf{f}$ as a vector space, it is automatically endowed with an $\mathbb{A}$-submodule ${ }_{\mathbb{A}} M(\lambda)={ }_{\mathbb{A}} \mathbf{f}$. We call this the integral form of $M(\lambda)$. We also have integral forms for the irreducible modules: we call the $\mathbb{A}$-submodule ${ }_{\mathbb{A}} V(\lambda)={ }_{\mathbb{A}} \mathbf{U} \xi_{-\lambda}\left(\right.$ resp. $\left.{ }_{\mathbb{A}}^{\omega} V(\lambda)={ }_{\mathbb{A}} \mathbf{U} \xi_{-\lambda}\right)$ the integral form of $V(\lambda)$ (resp. ${ }^{\omega} V(\lambda)$ ).

2.6. The canonical basis of $\mathbf{U}^{-}$. Let us recall some terminology. Let $R$ be a ring. A $\pi$-basis for a free $R^{\pi}$-module $M$ is a set $S \subset M$ such that there exists an $R^{\pi}$-basis $B$ for $M$ with $S=B \cup \pi B$. (We caution the reader that this is called a maximal $\pi$ basis in CHW2 .) We note that a $\pi$-basis of an $R^{\pi}$-module $M$ is an $R$-basis of $M$. The fundamental $\pi$-basis in this paper is the following.

Theorem 2.13 ([CHW2]). There is a $\pi$-basis $\mathcal{B}$ of $\mathbf{f}$ with the following properties:

(1) $\mathcal{B}$ is a $\pi$-basis of $\mathbb{A} \mathbf{f}$ over $\mathbb{A}$.

(2) Each $b \in \mathcal{B}$ is homogeneous.

(3) $\bar{b}=b$ for all $b \in \mathcal{B}$.

(4) For $\lambda \in X^{+}$, there is a subset $\mathcal{B}(\lambda)$ such that $\mathcal{B}(V(\lambda))=\left\{b \eta_{\lambda}: b \in \mathcal{B}(\lambda)\right\}$ is a $\pi$-basis of $V(\lambda)$, and if $b \in \mathcal{B} \backslash \mathcal{B}(\lambda), b^{-} \eta_{\lambda}=0$.

We note that $\left.\left.\mathcal{B}\right|_{\pi=1} \subset \mathbf{f}\right|_{\pi=1}$ is precisely the Lusztig-Kashiwara canonical basis. We shall need the following additional properties.

Lemma 2.14. Let $(\cdot, \cdot)$ be the polarization on $V(\lambda)$ (cf. [CHW2]). 
(1) We have $\left({ }_{\mathbb{A}} V(\lambda),{ }_{\mathbb{A}} V(\lambda)\right) \subset \mathbb{A}$.

(2) $\left(b^{-} \eta_{\lambda}, b^{\prime-} \eta_{\lambda}\right) \in \operatorname{sgn}(b) \delta_{b, b^{\prime}}+v \mathbb{Z}^{\pi}[v]$ for any $b, b^{\prime} \in \mathcal{B}$, where $\operatorname{sgn}(b) \in\{1, \pi\}$.

(3) For $b \in \mathcal{B}$, either $\rho(b)=b$ or $\rho(b)=\pi b$, so in particular $\rho(\mathcal{B})=\mathcal{B}$.

(4) $b \eta_{\lambda}=0$ if and only if $b \in \mathbb{A}_{\mathbb{A}} \mathbf{f} \theta_{i}^{(n)}$ for some $i \in I$ and $n \geq\langle i, \lambda\rangle+1$.

Proof. (1) and (2) follow from the proof of [L93, Proposition 19.3.3] and [CHW2, Proposition 6.1].

For (3), it is known that $\left.\mathcal{B}\right|_{\pi=1}$ is $\rho$-invariant ([L93, Theorem 14.4.3(c)]). To prove the more general fact, we utilize the map $\mathfrak{X}$ in Proposition 6.5 , for the convenience of the reader, we will restate the necessary facts here. By adjoining $\mathbf{t}=\sqrt{-1}$ to $\mathbb{Q}(v)^{\pi}$ and considering $\mathbf{f}[\mathbf{t}]=\mathbb{Q}(v)^{\pi}[\mathbf{t}] \otimes_{\mathbb{Q}(v)^{\pi}} \mathbf{f}$, there is a $\mathbb{Q}$-linear map $\mathfrak{X}: \mathbf{f}[\mathbf{t}] \rightarrow \mathbf{f}[\mathbf{t}]$ with $\mathfrak{X}(\pi)=-\pi$ and $\mathfrak{X}(b)=\mathbf{t}^{\ell(b)} b$ for some integer $\ell(b)$. Note that, in particular, $\mathfrak{X}\left(\epsilon_{+} b\right)=\mathbf{t}^{\ell(b)} \epsilon_{-} b$.

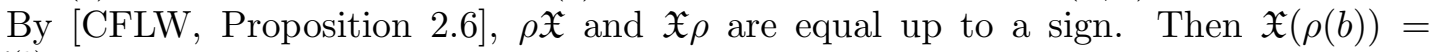
$\varsigma \mathbf{t}^{\ell(b)} \rho(b)$, where $\varsigma= \pm 1$. But then using $\rho$-invariance of the canonical basis when $\pi=1$,

$$
\mathbf{t}^{\ell(b)} \epsilon_{-} b=\mathfrak{X}\left(\epsilon_{+} b\right)=\mathfrak{X}\left(\rho\left(\epsilon_{+} b\right)\right)=\varsigma \mathbf{t}^{\ell(b)} \epsilon_{-} \rho(b) .
$$

Since $-\epsilon_{-}=\pi \epsilon_{-}, \rho\left(\epsilon_{-} b\right)=\epsilon_{-} b$ or $\epsilon_{-} \pi b$. Therefore, $\rho(b)=b$ or $\rho(b)=\pi b$, as claimed.

Finally, we note that $\mathfrak{X}\left(\mathbf{f}[\mathbf{t}] \theta_{i}^{n}\right) \subset \mathbf{f}[\mathbf{t}] \theta_{i}^{n}$, the statement of (4) holds for $\left.\mathcal{B}\right|_{\pi=1}$ (cf. [L93, Theorem 14.4.11]), and we have $\mathfrak{X}\left(\epsilon_{ \pm} b\right)=\mathbf{t}^{\ell(b)} \epsilon_{\mp} b$. Combining these facts proves (4) for $\left.\mathcal{B}\right|_{\pi=-1}$ and hence for $\mathcal{B}$.

\section{The MODIFIED QUANTUM COVERING GROUPS}

In this section, we will define the modified quantum group and systematically develop its fundamental properties. We also define a family of modules $\mathcal{F}$ which will be important in the subsequent sections.

3.1. The modified form. The modified quantum group may be defined as a direct sum of certain quotients of $\mathbf{U}$ (cf. [L93, Chapter 23]) but the essential description of this algebra may be given as follows.

Definition 3.1. The modified quantum covering group $\dot{\mathbf{U}}$ associated to the root datum $(Y, X, I, \cdot)$ is the associative $\mathbb{Q}(v)^{\pi}$-algebra without unit on symbols $x 1_{\lambda}$ and $1_{\lambda} x$ for $x \in \mathbf{U}$ and $\lambda \in X$ satisfying the relations

$$
\begin{gathered}
x 1_{\lambda} y 1_{\eta}=\delta_{\lambda, \eta+|y|}(x y) 1_{\lambda}, \quad x 1_{\lambda}=1_{\lambda+|x|} x \quad \text { for all homogeneous } x, y \in \mathbf{U}, \lambda \in X, \\
K_{\nu} 1_{\lambda}=v^{\langle\nu, \lambda\rangle} 1_{\lambda}, \quad J_{\nu} 1_{\lambda}=\pi^{\langle\nu, \lambda\rangle} 1_{\lambda} \quad \text { for all } \nu \in Y, \lambda \in X .
\end{gathered}
$$

Remark 3.2. A version of $\dot{\mathbf{U}}$ has already been defined in [CFLW, CW] in different ways. In [CFLW, Definition 4.2], the modified form is defined using generators $1_{\lambda}, E_{i} 1_{\lambda}$ and $F_{i} 1_{\lambda}$ for $i \in I$ and $\lambda \in X$ satisfying certain relations; it is straightforward to see that this is equivalent to our definition. In [CW, §6.1], a rank one modified form is defined using certain quotients of $\mathbf{U}$ in direct parallel to the definition in [L93, §23.1]. This construction can be generalized to higher rank, and results in an algebra isomorphic to our definition.

The algebra $\dot{\mathbf{U}}$ is naturally $\mathbf{U}$-bimodule under the following action: for $x, y, z \in \mathbf{U}$, we set

$$
x\left(y 1_{\lambda}\right) z=(x y z) 1_{\lambda-|z|} .
$$

We note the following commutation relations which may be deduced from [CHW1, Lemma 2.1.6] or [CW, Proposition 6.1]. 
Lemma 3.3. We have the following identities in $\dot{\mathbf{U}}$ :

$$
\begin{aligned}
& E_{i}^{(N)} 1_{\lambda} F_{i}^{(M)}=\sum_{t} \pi_{i}^{M N-\left(\begin{array}{c}
(t+1 \\
2
\end{array}\right)} F_{i}^{(M-t)}\left[\begin{array}{c}
M+N+\langle i, \lambda\rangle \\
t
\end{array}\right]_{v_{i}, \pi_{i}} 1_{\lambda+2 N+2 M-2 t} E_{i}^{(N-t)}, \\
& F_{i}^{(N)} 1_{\lambda} E_{i}^{(M)}=\sum_{t}(-1)^{t} \pi_{i}^{(M-t)(N-t)-t^{2}} E_{i}^{(M-t)}\left[\begin{array}{c}
M+N-\langle i, \lambda\rangle \\
t
\end{array}\right]_{v_{i}, \pi_{i}} 1_{\lambda-2 N-2 M+2 t} F_{i}^{(N-t)}, \\
& E_{i}^{(N)} F_{j}^{(M)} 1_{\lambda}=\pi^{M N p(i) p(j)} F_{j}^{(M)} E_{i}^{(N)} 1_{\lambda} \quad \text { if } i \neq j .
\end{aligned}
$$

The next proposition shows that the various algebra (anti)automorphisms on $\mathbf{U}$ we have previously defined have analogous (anti)automorphisms on $\dot{\mathbf{U}}$ which are compatible with the bimodule presentation.

\section{Proposition 3.4.}

(1) There exists a $\mathbb{Q}(v)^{\pi}$-algebra automorphism $\omega$ of $\dot{\mathbf{U}}$ satisfying

$$
\omega\left(x 1_{\lambda}\right)=\omega(x) 1_{-\lambda} .
$$

(2) There exists a $\mathbb{Q}(v)^{\pi}$-algebra antiautomorphism $\rho$ of $\mathbf{U}$ satisfying

$$
\rho\left(x 1_{\lambda}\right)=1_{-\lambda} \rho(x) .
$$

(3) There exists a $\mathbb{Q}^{\pi}$-algebra involution - of $\dot{\mathbf{U}}$ satisfying

$$
\overline{x 1_{\lambda}}=\bar{x} 1_{\lambda} .
$$

(4) There exists a $\mathbb{Q}^{\pi}$-algebra involution $\dagger$ of $\dot{\mathbf{U}}$ satisfying

$$
\left(x 1_{\lambda}\right)^{\dagger}=x^{\dagger} 1_{\lambda} .
$$

Proof. This follows as an elementary consequence of the existence of these maps on $\mathbf{U}$ and the definition of $\mathbf{U}$.

A $\dot{\mathbf{U}}$-module $M$ is called unital if for any $m \in M, m=\bigoplus_{\lambda \in X} 1_{\lambda} m$ with $1_{\lambda} m=0$ for all but finitely many $\lambda \in X$. As observed by Lusztig [L93], unital Uं-modules may naturally be viewed as weight $\mathbf{U}$-modules by interpreting the idempotent $1_{\lambda}$ as a projection onto the $\lambda$-weight space. More precisely, any unital $\mathbf{U}$-module $M$ is a weight $\mathbf{U}$-module under the action $x \cdot m=\sum_{\lambda \in X} x 1_{\lambda} m$, and similarly any weight $\mathbf{U}$-module $M$ is a unital $\dot{\mathbf{U}}$-module via $x 1_{\lambda} m=x m_{\lambda}$, where $m_{\lambda}$ is the orthogonal projection of $m$ to the $M_{\lambda}$ weight space.

3.2. Integral form. Suppose $M$ and $M^{\prime}$ are free $\mathbb{Q}(v)^{\pi}$-modules with $\pi$-bases $S \subset M$ and $S^{\prime} \subset M^{\prime}$. The $S \otimes S=\left\{s \otimes s \mid s \in S, s^{\prime} \in S^{\prime}\right\}$ is clearly a $\pi$-basis for $M \otimes M^{\prime}$. However, we note the map $S \times S^{\prime} \rightarrow S \otimes S^{\prime}$ given by $\left(s, s^{\prime}\right) \mapsto s \otimes s^{\prime}$ is not a bijection, since clearly $s \otimes s^{\prime}=(\pi s) \otimes\left(\pi s^{\prime}\right)$. We will occasionally need an honest index set, so let $\sim$ be the equivalence relation on $S \times S^{\prime}$ given by $\left(\pi s, s^{\prime}\right) \sim\left(s, \pi s^{\prime}\right)$. Then we set

$$
S \times \pi S^{\prime}=S \times S^{\prime} / \sim \text {. }
$$

From the triangular decomposition of $\mathbf{U}, \mathbf{U}$ has a $\pi$-basis consisting of elements of the form $b^{+} J_{\mu} K_{\nu} b^{-}$where $\left(b, b^{\prime}\right) \in \mathcal{B} \times_{\pi} \mathcal{B}$ and $\mu, \nu \in Y$. Since

$$
\left(b^{+} J_{\mu} K_{\nu} b^{\prime-}\right) 1_{\lambda}=\pi^{\left\langle\mu, \lambda_{1}\right\rangle} v^{\left\langle\nu, \lambda_{1}\right\rangle} b^{+} 1_{\lambda_{1}} b^{\prime-},
$$

where $\lambda_{1}=\lambda-\left|b^{\prime}\right|$, we see that $\left\{b^{+} 1_{\lambda} b^{\prime-}:\left(b, b^{\prime}\right) \in \mathcal{B} \times_{\pi} \mathcal{B}\right\}$ forms a $\pi$-basis of $\dot{\mathbf{U}}$. Similarly, $\left\{b^{-} 1_{\lambda} b^{\prime+}:\left(b, b^{\prime}\right) \in \mathcal{B} \times_{\pi} \mathcal{B}\right\}$ forms a $\pi$-basis of $\dot{\mathbf{U}}$. In fact, these elements span an integral form of $\dot{\mathbf{U}}$.

\section{Lemma 3.5.}


(1) The $\mathbb{A}$-submodule of $\dot{\mathbf{U}}$ spanned by the elements $x^{+} 1_{\lambda} x^{\prime-}$ (with $x, x^{\prime} \in{ }_{\mathbb{A}} \mathbf{f}$ ) coincides with the $\mathbb{A}$-submodule of $\dot{\mathbf{U}}$ spanned by the elements $x^{-} 1_{\lambda} x^{\prime+}$ (with $x, x^{\prime} \in$ $\left.{ }_{\mathbb{A}} \mathbf{f}\right)$. We denote it by $\mathbb{A}_{\mathbf{U}}$.

(2) The elements $\left\{b^{+} 1_{\lambda} b^{\prime-}:\left(b, b^{\prime}\right) \in \mathcal{B} \times_{\pi} \mathcal{B}\right\}$ (resp. $\left\{b^{-} 1_{\lambda} b^{\prime+}:\left(b, b^{\prime}\right) \in \mathcal{B} \times_{\pi} \mathcal{B}\right\}$ ) form a $\pi$-basis of ${ }_{\mathbb{A}} \dot{\mathbf{U}}$.

(3) ${ }_{\mathbb{A}} \dot{\mathbf{U}}$ is a $\mathbb{A}$-subalgebra of $\dot{\mathbf{U}}$ which is generated by the elements $E_{i}^{(n)} 1_{\lambda}$ and $F_{i}^{(n)} 1_{\lambda}$ for $i \in I, n \geq 0$, and $\lambda \in X$.

Proof. Recall that ${ }_{\mathbb{A}} \mathbf{f}$ is the $\mathbb{Z}\left[v^{ \pm 1}\right]^{\pi}$ generated by $\theta_{i}^{(n)}$ for $i \in I$ and $n \geq 0$, and so repeated application of Lemma 3.3 implies (1) and (3). (2) follows from the triangular decomposition of $\dot{\mathbf{U}}$.

3.3. Tensor modules of $\dot{U}$. The algebra $\dot{\mathbf{U}}$ does not admit a natural co-product, as any candidate would require an infinite sum (cf. [L93, §23.1.5]). However, since unital modules and weight modules are equivalent, the Hopf structure on $\mathbf{U}$ imposes a unital Ü-module structure on the tensor product of weight modules. In the following, we will occasionally need to consider these tensor products as modules under different coproducts of $\mathbf{U}$. To that end, we will now relate the coproducts from $\$ 2.4$,

For $s \in\{1,2,3,4\}$, let $\Delta_{s}^{\omega}=\left(\omega^{-1} \otimes \omega^{-1}\right) \circ \Delta_{s} \circ \omega$ and $\Delta_{s}^{\omega^{-1}}=(\omega \otimes \omega) \circ \Delta_{s} \circ \omega^{-1}$. Let $\overline{\Delta_{s}}={ }^{-} \circ \Delta_{s} \circ-$, where ${ }^{-}=-\otimes^{-}: \mathbf{U} \otimes \mathbf{U} \rightarrow \mathbf{U} \otimes \mathbf{U}$. Also, let ${ }^{\tau} \Delta_{s}$ be the composition of $\Delta_{s}$ with the automorphism $\tau: \mathbf{U} \otimes \mathbf{U} \rightarrow \mathbf{U} \otimes \mathbf{U}$ given by $x \otimes y \mapsto \pi^{p(x) p(y)} y \otimes x$. In particular, we note that ${ }^{\tau} \bar{\Delta}_{s}^{\omega}$ is a well defined symbol, since $\tau,{ }^{-} \otimes^{-}$and $\omega \otimes \omega$ all commute, and $\omega$ and ${ }^{-}$commute.

Lemma 3.6. We have the following identifications:

$$
\begin{gathered}
\Delta_{2}={ }^{\tau} \Delta_{1}^{\omega} \\
\Delta_{3}={ }^{\tau} \bar{\Delta}_{1} \\
\Delta_{4}={ }^{\tau} \Delta_{3}^{\omega^{-1}}=\bar{\Delta}_{1}^{\omega^{-1}}
\end{gathered}
$$

Proof. First, let us assume all the equalities but ${ }^{\tau} \Delta_{3}^{\omega^{-1}}=\bar{\Delta}_{1}^{\omega^{-1}}$. Then we see that

$$
{ }^{\tau} \Delta_{3}^{\omega}=\tau \circ \omega \otimes \omega \circ \tau \circ-\circ \Delta_{1} \circ-\circ \omega^{-1} \text {. }
$$

Since $\tau$ is an involution commuting with ${ }^{-}$and $\omega \otimes \omega$, we see the desired equality holds.

It remains to check the other equalities. Since all of the maps on the right-hand side are compositions of algebra homomorphisms, we may simply check on generators. The lemma is clear for $K_{\mu}$ and $J_{\mu}$, so it suffices to check the equalities on $E_{i}$ and $F_{i}$.

We compute

$$
\begin{aligned}
\Delta_{1}^{\omega}\left(E_{i}\right) & =E_{i} \otimes \tilde{K}_{i}+1 \otimes E_{i}, & \Delta_{1}^{\omega}\left(F_{i}\right) & =F_{i} \otimes \tilde{J}_{i}+\tilde{K}_{-i} \otimes F_{i}, \\
\bar{\Delta}_{1}\left(E_{i}\right) & =E_{i} \otimes 1+\tilde{K}_{-i} \otimes E_{i}, & \bar{\Delta}_{1}\left(F_{i}\right) & =F_{i} \otimes \tilde{J}_{i} \tilde{K}_{i}+1 \otimes F_{i}, \\
\Delta_{3}^{\omega}\left(E_{i}\right) & =E_{i} \otimes \tilde{J}_{i}+\tilde{K}_{-i} \otimes E_{i}, & \Delta_{3}^{\omega}\left(F_{i}\right) & =F_{i} \otimes \tilde{K}_{i}+1 \otimes F_{i} .
\end{aligned}
$$

Applying $\tau$ to these identities completes the proof of the lemma.

We note the following consequence of the lemma.

Lemma 3.7. For any $\mathbf{U}$-modules $M, M^{\prime}$, there is a $\mathbf{U}$-module isomorphism

$$
\tau:{ }^{\omega}\left(M \otimes{ }_{4} M^{\prime}\right) \rightarrow{ }^{\omega} M^{\prime} \otimes{ }^{\omega} M
$$

given by $\tau(x \otimes y): \pi^{p(y) p(x)} y \otimes x$. 
Proof. Let $u \in \mathbf{U}$ and suppose $\Delta_{4}(\omega(u))=\sum u_{1} \otimes u_{2}$ with $u_{1}, u_{2}$ homogeneous. Then

$$
\begin{aligned}
\tau\left(\Delta_{4}(\omega(u))(x \otimes y)\right) & =\tau\left(\sum \pi^{p(x) p\left(u_{2}\right)} u_{1} x \otimes u_{2} y\right) \\
& =\sum \pi^{p(x) p\left(u_{2}\right)+p\left(u_{1} x\right) p\left(u_{2} y\right)} \sum u_{2} y \otimes u_{1} x \\
& =\left(\sum \pi^{p\left(u_{2}\right) p\left(u_{1}\right)} \omega^{-1}\left(u_{2}\right) \otimes \omega^{-1}\left(u_{1}\right)\right) \tau(x \otimes y) \\
& =\tau \circ\left(\omega^{-1} \otimes \omega^{-1}\right) \circ \Delta_{4}(\omega(u)) \tau(x \otimes y) \\
& =\Delta(u) \tau(x \otimes y) .
\end{aligned}
$$

3.4. The Verma identification. Recall that the Verma module $M(\lambda)$ is isomorphic to f as a $\mathbb{Q}(v)^{\pi}$-module. We shall now describe a generalization of this identification to $\dot{\mathbf{U}}$.

Let $\lambda, \lambda^{\prime} \in X$. As discussed in $\$ 3.3$, the U-module $M(\lambda) \otimes{ }^{\omega} M\left(\lambda^{\prime}\right)$ is naturally a Ü-module. As it will often be convenient, we will use the $\mathbb{Q}(v)^{\pi}$-module identification

$$
M(\lambda) \otimes{ }^{\omega} M\left(\lambda^{\prime}\right)=\mathbf{f} \otimes \mathbf{f} .
$$

From the triangular decomposition of $\dot{\mathbf{U}}$, the elements $b^{+} b^{\prime-} 1_{\zeta}$ with $\left(b, b^{\prime}\right) \in \mathcal{B} \times_{\pi} \mathcal{B}$ comprise a $\pi$-basis of $\dot{\mathbf{U}}$. Likewise, the canonical basis of $\mathbf{U}^{-} \cong \mathbf{f}$ induces a $\pi$-basis $\mathcal{B} \otimes \mathcal{B}=\left\{b \otimes b^{\prime} \mid\left(b, b^{\prime}\right) \in \mathcal{B} \times_{\pi} \mathcal{B}\right\}$ on $\mathbf{f} \otimes \mathbf{f}$.

If $\zeta=\lambda-\lambda^{\prime}$, then since a $\pi$-basis is a $\mathbb{Q}(v)$-basis, we have

$$
b^{+} b^{\prime-} 1_{\zeta}(1 \otimes 1)=b^{+}\left(b^{\prime} \otimes 1\right)=\pi^{p\left(b^{\prime}\right) p(b)} b^{\prime} \otimes b+\sum c_{b_{1}^{\prime}, b_{1}} b_{1}^{\prime} \otimes b_{1},
$$

where $c_{b_{1}^{\prime}, b_{1}} \in \mathbb{Q}(v)$ are constants, $\nu=|b|$, and the sum is over $\left(b_{1}, b_{1}^{\prime}\right) \in \mathcal{B} \times_{\pi} \mathcal{B}$ such that $\mathrm{ht}\left|b_{1}\right|<\mathrm{ht}|b|, \mathrm{ht}\left|b_{1}^{\prime}\right|<\mathrm{ht}\left|b^{\prime}\right|$ and $b_{1}^{\prime}$ belongs to the $\dot{\mathbf{U}}$-submodule of $M(\lambda)$ generated by $b^{\prime}$. Moreover, since $\mathcal{B} \subset{ }_{\mathbb{A}} \mathbf{U}$, the left-hand side of (3.2) lies in ${ }_{\mathbb{A}} \mathbf{f} \otimes_{\mathbb{A} A} \mathbf{f}$ and hence we have $c_{b_{1}^{\prime}, b_{1}} \in \mathbb{Z}\left[v, v^{-1}\right]$.

Similarly, from the triangular decomposition of $\dot{\mathbf{U}}$, the elements $b^{-} b^{\prime+} 1_{\zeta}$ comprise a $\pi$-basis of $\dot{\mathbf{U}}$. If $\zeta=\lambda-\lambda^{\prime}$, then

$$
b^{-} b^{\prime+} 1_{\zeta}(1 \otimes 1)=b^{-}\left(1 \otimes b^{\prime}\right)=b \otimes b^{\prime}+\sum c_{b_{1}, b_{1}^{\prime}} b_{1} \otimes b_{1}^{\prime},
$$

where $c_{b_{1}, b_{1}^{\prime}}^{\prime} \in \mathbb{Z}\left[v, v^{-1}\right]$ are constants and the sum is over $b_{1}, b_{1}^{\prime} \in \mathcal{B} \times_{\pi} \mathcal{B}$ such that $\mathrm{ht}\left|b_{1}\right|<\mathrm{ht}|b|, \mathrm{ht}\left|b_{1}^{\prime}\right|<\mathrm{ht}\left|b^{\prime}\right|$ and $b_{1}^{\prime}$ belongs to the $\dot{\mathbf{U}}$-submodule of ${ }^{\omega} M\left(\lambda^{\prime}\right)$ generated by $b^{\prime}$.

In either case, note that the transition matrix between the $\pi$-basis $\mathcal{B} \otimes \mathcal{B}$ and the elements $\left\{b^{-} b^{\prime+} 1_{\zeta}(1 \otimes 1) \mid b, b^{\prime} \in \mathcal{B} \times_{\pi} \mathcal{B}\right\}$ is upper unitriangular with entries in $\mathbb{Z}\left[v, v^{-1}\right]$, hence the latter is also a $\pi$-basis. In particular, we see that the $\mathbb{Q}(v)^{\pi}$-linear map

$$
\check{\partial}_{\lambda, \lambda^{\prime}}: \dot{\mathbf{U}} 1_{\lambda-\lambda^{\prime}} \rightarrow M(\lambda) \otimes{ }^{\omega} M\left(\lambda^{\prime}\right), \quad u \mapsto u(1 \otimes 1)
$$

is an isomorphism. Similarly, the $\mathbb{A}$-linear map

$$
{ }_{\mathbb{A}} \partial_{\lambda, \lambda^{\prime}}:{ }_{\mathbb{A}} \dot{\mathbf{U}} 1_{\lambda-\lambda^{\prime}} \rightarrow{ }_{\mathbb{A}} M(\lambda) \otimes_{\mathbb{A}} \stackrel{\omega}{\mathbb{A}} M\left(\lambda^{\prime}\right), \quad u \mapsto u(1 \otimes 1)
$$

is an isomorphism. 
3.5. The family $\mathcal{F}$. The following particular family of $\mathbf{U}$-modules will be of critical importance later on. Let $\mathcal{F}=\left\{N\left(\lambda, \lambda^{\prime}\right) \mid \lambda, \lambda^{\prime} \in X^{+}\right\}$, where

$$
N\left(\lambda, \lambda^{\prime}\right)=V(\lambda) \otimes_{\mathbb{Q}(q)^{\pi}}{ }^{\omega} V\left(\lambda^{\prime}\right), \quad{ }_{\mathbb{A}} N\left(\lambda, \lambda^{\prime}\right)={ }_{\mathbb{A}} V(\lambda) \otimes_{\mathbb{A}}\left(\stackrel{\mathbb{A}}{\omega}^{\omega} V\left(\lambda^{\prime}\right)\right) .
$$

Denote by $N_{s}\left(\lambda, \lambda^{\prime}\right)$ (resp. ${ }_{\mathbb{A}} N_{s}\left(\lambda, \lambda^{\prime}\right)$ ) the $\mathbf{U}$-module (resp. ${ }_{\mathbb{A}} \mathbf{U}$-module) obtained by acting on $N\left(\lambda, \lambda^{\prime}\right)$ (resp. $\mathbb{A} N\left(\lambda, \lambda^{\prime}\right)$ ) via $\Delta_{s}$. When there can be no confusion, we will shorten notation to $N\left(\lambda, \lambda^{\prime}\right)=N_{3}\left(\lambda, \lambda^{\prime}\right)$ and ${ }_{\mathbb{A}} N\left(\lambda, \lambda^{\prime}\right)={ }_{\mathbb{A}} N_{3}\left(\lambda, \lambda^{\prime}\right)$.

The module structures on $N_{3}\left(\lambda, \lambda^{\prime}\right)$ and $N_{4}\left(\lambda, \lambda^{\prime}\right)$ are quite closely related, as demonstrated by the following lemma.

Lemma 3.8. The linear isomorphism $N_{3}\left(\lambda, \lambda^{\prime}\right) \rightarrow N_{4}\left(\lambda, \lambda^{\prime}\right)$ given by

$$
x \otimes y \mapsto \pi^{\langle\tilde{\nu}, \lambda\rangle} x \otimes y \quad \text { for all } x \in V(\lambda), y \in{ }^{\omega} V\left(\lambda^{\prime}\right)_{\nu^{\prime}-\lambda^{\prime}}, \nu \in \mathbb{N}[I],
$$

is a $\mathbf{U}$-module isomorphism.

Proof. This is elementary to verify using the definitions.

Now let us examine the relationship between $N\left(\lambda, \lambda^{\prime}\right)$ and $M(\lambda) \otimes{ }^{\omega} M\left(\lambda^{\prime}\right)$. Let $\zeta \in X$ and let $a=\sum_{i} a_{i} i, a^{\prime}=\sum_{i} a_{i}^{\prime} i \in \mathbb{N}[I]$ such that $\langle i, \zeta\rangle=a_{i}^{\prime}-a_{i}$ for all $i \in I$. We define the ideals

$$
\begin{aligned}
P\left(\zeta, a, a^{\prime}\right) & =\sum_{i, n>a_{i}} \dot{\mathbf{U}} F_{i}^{(n)} 1_{\zeta}+\sum_{i, n>a_{i}^{\prime}} \dot{\mathbf{U}} E_{i}^{(n)} 1_{\zeta}, \\
\mathbb{A} P\left(\zeta, a, a^{\prime}\right) & =\sum_{i, n>a_{i}} \dot{\mathbf{U}} F_{i}^{(n)} 1_{\zeta}+\sum_{i, n>a_{i}^{\prime}} \dot{\mathbf{U}} E_{i}^{(n)} 1_{\zeta} .
\end{aligned}
$$

Let $\lambda, \eta \in X^{+}$and set $a_{i}=\langle i, \lambda\rangle, a_{i}^{\prime}=\langle i, \eta\rangle$ for all $i \in I$. Set $\xi=\eta-\lambda, a=$ $\sum_{i} a_{i} i \in \mathbb{N}[I]$, and $a^{\prime}=\sum_{i} a_{i}^{\prime} \in \mathbb{N}[I]$. Let $\mathcal{T}$ (resp. $\mathcal{T}^{\prime}$ ) be the kernel of the canonical homomorphism of $\mathbf{U}$-modules $\mathbf{f}=M(\lambda) \rightarrow V(\lambda)$ (resp. $\mathbf{f}={ }^{\omega} M\left(\lambda^{\prime}\right) \rightarrow{ }^{\omega} V\left(\lambda^{\prime}\right)$ ). Then by Theorem 2.13 (4) and Lemma $2.14(4), \mathcal{T}$ (resp. $\mathcal{T}^{\prime}$ ) is generated by $b \in \mathcal{B}$ such that $b \in{ }_{\mathbb{A}} \mathbf{f} \theta_{i}^{(n)}$ for some $n \geq a_{i}$ (resp. $n \geq a_{i}^{\prime}$ ).

Then taking tensor products, we obtain the surjective homomorphism

$$
M(\lambda) \otimes{ }^{\omega} M\left(\lambda^{\prime}\right) \rightarrow N\left(\lambda, \lambda^{\prime}\right) .
$$

The kernel of this map is the subspace $\mathcal{T} \otimes \mathbf{f}+\mathbf{f} \otimes \mathcal{T}^{\prime}$.

Let $\mathcal{D}(\lambda)=\mathcal{B} \backslash \mathcal{B}(\lambda)$. Now by the triangular decomposition, the description of $\mathcal{T}$, and (3.2), $\partial_{\lambda, \lambda^{\prime}}$ maps the subspace

$$
\sum_{b \in D(\lambda), b^{\prime} \in \mathcal{B}} \mathbb{Q}(v)^{\pi} b^{\prime+} b^{-} 1_{\zeta}=\sum_{i, n>a_{i}} \dot{\mathbf{U}} F_{i}^{(n)} 1_{\zeta}
$$

onto the subspace

$$
\sum_{b \in D(\lambda), b^{\prime} \in \mathcal{B}} \mathbb{Q}(v)^{\pi} b \otimes b^{\prime}=\mathcal{T} \otimes \mathbf{f} .
$$

Similarly by the triangular decomposition, the description of $\mathcal{T}^{\prime}$, and $(\sqrt{3.3}), \check{\partial}_{\lambda, \lambda^{\prime}}$ maps the subspace

onto the subspace

$$
\sum_{b \in \mathcal{B}, b^{\prime} \in D\left(\lambda^{\prime}\right)} \mathbb{Q}(v)^{\pi} b^{-} b^{\prime+} 1_{\zeta}=\sum_{i, n>a_{i}} \dot{\mathbf{U}} E_{i}^{(n)} 1_{\zeta}
$$

$$
\sum_{b \in \mathcal{B}, b^{\prime} \in D\left(\lambda^{\prime}\right)} \mathbb{Q}(v)^{\pi} b \otimes b^{\prime}=\mathbf{f} \otimes \mathcal{T}^{\prime} .
$$

We note that replacing everything with the integral form in the preceding argument does not effect the argument, and thus we have proven the following. 
Proposition 3.9. The map $u \mapsto u\left(\eta_{\lambda} \otimes \xi_{-\lambda^{\prime}}\right)$ defines a surjective linear map $\dot{\mathbf{U}} \rightarrow$ $N\left(\lambda, \lambda^{\prime}\right)$ with kernel equal to $P\left(\zeta, a, a^{\prime}\right)$. Moreover, restriction of this map to ${ }_{\mathbb{A}} \dot{\mathbf{U}}$ gives a surjective linear map $\mathbb{A}_{\mathbb{U}} \dot{\mathbf{U}} \rightarrow_{\mathbb{A}} N\left(\lambda, \lambda^{\prime}\right)$ with kernel equal to ${ }_{\mathbb{A}} P\left(\zeta, a, a^{\prime}\right)$.

We now note that each module in $\mathcal{F}$ comes equipped with a $\pi$-basis. Indeed, the elements

$$
\mathcal{B}\left(\lambda, \lambda^{\prime}\right)=\left\{b^{-} \eta_{\lambda} \otimes b^{+} \xi_{-\lambda^{\prime}}: b \times b^{\prime} \in B(\lambda) \times{ }_{\pi} B\left(\lambda^{\prime}\right)\right\}
$$

form a $\pi$-basis of $N\left(\lambda, \lambda^{\prime}\right)$. They generate a $\mathbb{Z}^{\pi}[v]$-submodule $\mathcal{L}=\mathcal{L}\left(\lambda, \lambda^{\prime}\right)$ and a $\mathbb{A}$ submodule $_{\mathbb{A}} \mathcal{L}$.

\section{Canonical Bases}

In this section, we will construct bar-invariant canonical bases for the modules in $\mathcal{F}$ and show that they are mutually compatible with respect to a family of maps. This enables us to construct a "projective limit" of these canonical bases, and this limit is a canonical basis for $\dot{\mathbf{U}}$.

4.1. Bar involution for $\mathcal{F}$. Recall the quasi-R-matrix $\Theta=\sum_{\nu \in \mathbb{N}[I]} \Theta_{\nu}$ from Proposition 2.9. We recall in particular that $\Theta$ lives in a suitable completion of $\mathbf{U} \otimes \mathbf{U}$, and that (in this completion) we have

$$
\Delta(u) \Theta=\Theta \bar{\Delta}(u), \quad \Theta \bar{\Theta}=1 .
$$

where $^{-}: \mathbf{U} \otimes \mathbf{U} \rightarrow \mathbf{U} \otimes \mathbf{U}$ is the map $\overline{x \otimes y}=\bar{x} \otimes \bar{y}$.

Let $M$ and $M^{\prime}$ be weight modules such that ${ }^{\omega} M \in \mathcal{O}$ or $M^{\prime} \in \mathcal{O}$. Then since $\Theta_{\nu} \in$ $\mathbf{U}_{\nu}^{+} \otimes \mathbf{U}_{\nu}^{-}$, given any $x \in M \otimes M^{\prime}$, we must have $\Theta_{\nu} x=0$ for all but a finite number of $\nu \in \mathbb{N}[I]$. Then by regarding $M \otimes M^{\prime}$ as a $\mathbf{U} \otimes \mathbf{U}$-module, $\Theta$ defines a linear map $\Theta: M \otimes M^{\prime} \rightarrow M \otimes M^{\prime}$ by $m \otimes m^{\prime} \mapsto \sum \Theta_{\nu}\left(m \otimes m^{\prime}\right)$. This is well-defined because only finitely many terms may be non-zero, and we see that

$$
\Delta(u) \Theta\left(m \otimes m^{\prime}\right)=\Theta\left(\overline{\Delta(\bar{u})} m \otimes m^{\prime}\right) .
$$

In particular, suppose that $M$ and $M^{\prime}$ are equipped with bar-involutions ${ }^{-}: M \rightarrow M$ and - : $M^{\prime} \rightarrow M^{\prime}$ such that $\overline{u m}=\overline{u m}$ and $\overline{u m^{\prime}}=\overline{u m^{\prime}}$ for all $u \in \mathbf{U}, m \in M$ and $m^{\prime} \in M^{\prime}$. Then

$$
\Delta(u) \Theta\left(m \otimes m^{\prime}\right)=\Theta\left(\overline{\Delta(\bar{u}) \bar{m} \otimes \bar{m}^{\prime}}\right)
$$

where ${ }^{-}={ }^{-} \otimes^{-}: M \otimes M^{\prime} \rightarrow M \otimes M^{\prime}$.

Theorem 4.1. Let $\lambda, \lambda^{\prime} \in X$ and consider the Verma modules $M(\lambda)$ and $M\left(\lambda^{\prime}\right)$. Set $M=M(\lambda) \in \mathcal{O}$ and $M^{\prime}={ }^{\omega} M\left(\lambda^{\prime}\right) \in \mathcal{C}$. Then $\Theta$ is a well defined map on $M \otimes M^{\prime}$ which leaves stable the $\mathbb{A}$-submodule $\mathbb{\mathbb { A }} M(\lambda) \otimes_{\mathbb{A}}\left(\underset{\mathbb{A}}{\omega} M\left(\lambda^{\prime}\right)\right)$.

Proof. The proof of this result is essentially the same as in [L93, Prop 24.1.4], but we shall state it here for completeness.

Since the ambient space of $M(\lambda)$ and $M\left(\lambda^{\prime}\right)$ is $\mathbf{f}$, there are well defined maps ${ }^{-}: M \rightarrow M$ and ${ }^{-}: M^{\prime} \rightarrow M^{\prime}$. On the other hand, $M(\lambda)$ and ${ }^{\omega} M\left(\lambda^{\prime}\right)$ may be identified as $\mathbf{U}$-modules with certain quotients of $\mathbf{U}$ such that the bar involution on $\mathbf{U}$ induces those on $M$ and $M^{\prime}$, whence $\overline{u m}=\overline{u m}$ and $\overline{u m^{\prime}}=\overline{u m^{\prime}}$ for $u \in \mathbf{U}, m \in M$ and $m^{\prime} \in M^{\prime}$. Then we set $-{ }^{-} \otimes^{-}: M \otimes M^{\prime} \rightarrow M \otimes M^{\prime}$.

Now let us identify $M(\lambda)$ and ${ }^{\omega} M\left(\lambda^{\prime}\right)$ with $\mathbf{f}$. We note that by definition, $\overline{1}=1$ in $M$ and $M^{\prime}$. Moreover, $\Theta(1 \otimes 1)=1 \otimes 1$. Then we have

$$
u(1 \otimes 1)=\Theta(\overline{\bar{u}(1 \otimes 1)}) .
$$


Since the ambient space of $\mathbb{A} M(\lambda)$ and ${ }_{\mathbb{A}}^{\omega} M\left(\lambda^{\prime}\right)$ is $\mathbb{A} \mathbf{f}$, which is bar-invariant, we see that $\mathbb{A}_{\mathbb{A}} M(\lambda) \otimes_{\mathbb{A}}\left({ }_{\mathbb{A}}^{\omega} M\left(\lambda^{\prime}\right)\right)$ is stable under - . Take $x \in{ }_{\mathbb{A}} M(\lambda) \otimes_{\mathbb{A}}\left({ }_{\mathbb{A}}^{\omega} M\left(\lambda^{\prime}\right)\right)$, and set $x^{\prime}=\bar{x} \in_{\mathbb{A}} M(\lambda) \otimes_{\mathbb{A}}\left(\underset{\mathbb{A}}{\omega} M\left(\lambda^{\prime}\right)\right)$.

On the other hand, the isomorphism ${ }_{\mathbb{A}} \dot{\mathbf{U}} 1_{\lambda^{\prime}-\lambda} \rightarrow_{\mathbb{A}} M(\lambda) \otimes_{\mathbb{A}}\left({ }_{\mathbb{A}}^{\omega} M\left(\lambda^{\prime}\right)\right)$ implies there is a $u^{\prime} \in{ }_{\mathbb{A}} \dot{\mathbf{U}} 1_{\lambda-\lambda^{\prime}}$ such that $u^{\prime}(1 \otimes 1)=x^{\prime}$. There is also a $u=\overline{u^{\prime}} \in{ }_{\mathbb{A}} \dot{\mathbf{U}} 1_{\lambda-\lambda^{\prime}}$. Therefore,

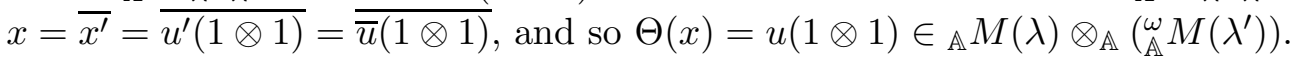

This immediately implies the following corollary.

Corollary 4.2. The map $\Theta$ leaves stable the $\mathbb{A}$-submodule $\mathbb{A}_{\mathbb{A}} N\left(\lambda, \lambda^{\prime}\right)$ (resp. $\mathbb{A}_{\mathbb{A}} N^{\prime}\left(\lambda, \lambda^{\prime}\right)$ ) of $N\left(\lambda, \lambda^{\prime}\right)$ (resp. $\left.N^{\prime}\left(\lambda, \lambda^{\prime}\right)\right)$.

Let $-: V\left(\lambda^{\prime}\right) \rightarrow V\left(\lambda^{\prime}\right)$ be the unique $\mathbb{Q}^{\pi}$-linear involution such that $\overline{u \eta_{\lambda^{\prime}}}=\bar{u} \eta_{\lambda^{\prime}}$ for all $u \in \mathbf{U}$; similarly, let $-:{ }^{\omega} V(\lambda) \rightarrow{ }^{\omega} V(\lambda)$ be the unique $\mathbb{Q}^{\pi}$-linear involution such that $\overline{u \xi_{-\lambda}}=\bar{u} \xi_{-\lambda}$ for all $u \in \mathbf{U}$. Let $-=^{-} \otimes: N\left(\lambda, \lambda^{\prime}\right) \rightarrow N\left(\lambda, \lambda^{\prime}\right)$.

Since the maps $\Theta,^{-}: N\left(\lambda, \lambda^{\prime}\right) \rightarrow N\left(\lambda, \lambda^{\prime}\right)$ are well-defined, let $\Psi=\Theta \circ-$. Then note that

$$
\Psi\left(\Delta(u) m \otimes m^{\prime}\right)=\Theta\left(\overline{\Delta(u) m \otimes m^{\prime}}\right)=\Delta(\bar{u}) \Theta\left(\bar{m} \otimes \overline{m^{\prime}}\right)=\Delta(\bar{u}) \Psi\left(m \otimes m^{\prime}\right),
$$

and that $\Psi^{2}=1$, from whence we call $\Psi$ the bar-involution on $N\left(\lambda, \lambda^{\prime}\right)$.

4.2. General lemma on semi-linear equations. We will now present an analogue of [L93, §24.2] in the covering setting.

Lemma 4.3. Let $H$ be a set with a partial order $\leq$ such that for any $h \leq h^{\prime}$ in $H$, the set $\left\{h^{\prime \prime}: h \leq h^{\prime \prime} \leq h^{\prime}\right\}$ is finite. Assume that for each $h \leq h^{\prime}$ in $H$, there exists an element $r_{h, h^{\prime}} \in \mathbb{A}$ such that $r_{h, h}=1$ and

$$
\sum_{h^{\prime \prime} ; h \leq h^{\prime \prime} \leq h^{\prime}} \bar{r}_{h, h^{\prime \prime}} r_{h^{\prime \prime}, h^{\prime}}=\delta_{h, h^{\prime}}
$$

for all $h \leq h^{\prime} \in H$.

Then there is a unique family of elements $p_{h, h^{\prime}} \in \mathbb{Z}^{\pi}[v]$ defined for all $h \leq h^{\prime} \in H$ such that $p_{h, h}=1, p_{h, h^{\prime}} \in v \mathbb{Z}^{\pi}[v]$ for all $h<h^{\prime}$ in $H$, and

$$
p_{h, h^{\prime}}=\sum_{h^{\prime \prime} ; h \leq h^{\prime \prime} \leq h^{\prime}} \bar{p}_{h, h^{\prime \prime}} r_{h^{\prime \prime}, h^{\prime}}
$$

for all $h \leq h^{\prime} \in H$.

Proof. For $h \leq h^{\prime}$ in $H$, denote by $d\left(h, h^{\prime}\right)$ the maximum length of a chain $h=h_{0}<h_{1}<$ $\ldots<h_{\ell}=h^{\prime} \in H$. Note that $d\left(h, h^{\prime}\right)<\infty$ by our assumption on $H$. For any $n \geq 0$, let $P_{n}$ be the assertion of the lemma restricted to those $h \leq h^{\prime}$ such that $d\left(h, h^{\prime}\right) \leq n$ (and note that all the assertions make sense under this restriction. We will prove $P_{n}$ by induction.

First note $P_{0}$ is trivial and assume that $n \geq 1$. Let $h \leq h^{\prime}$. If $d\left(h, h^{\prime}\right)<n$ then $p_{h, h^{\prime}}$ is defined by $P_{n-1}$. If $d\left(h, h^{\prime}\right)=n$, then $q=\sum_{h^{\prime \prime} ; h \leq h^{\prime \prime}<h^{\prime}} \bar{p}_{h, h^{\prime \prime}} r_{h^{\prime \prime}, h^{\prime}}$ is defined. 
First, we shall show that $q+\bar{q}=0$. Indeed, using $P_{n-1}$ and the assumptions of the lemma,

$$
\begin{aligned}
q+\bar{q} & =\sum_{h_{1} ; h \leq h_{0}<h^{\prime}} \bar{p}_{h, h_{0}} r_{h_{0}, h^{\prime}}+\sum_{h_{1} ; h \leq h_{1}<h^{\prime}} p_{h, h_{1}} \bar{r}_{h_{1}, h^{\prime}} \\
& =\sum_{h_{0}, h_{1} ; h \leq h_{0}<h_{1}=h^{\prime}} \bar{p}_{h, h_{0}} r_{h_{0}, h_{1}} \bar{r}_{h_{1}, h^{\prime}}+\sum_{h_{0}, h_{1} ; h \leq h_{0} \leq h_{1}<h^{\prime}} \bar{p}_{h, h_{0}} r_{h_{0}, h_{1}} \bar{r}_{h_{1}, h^{\prime}} \\
& =\sum_{h_{0}, h_{1} ; h \leq h_{0} \leq h_{1} \leq h^{\prime} ; h_{0}<h^{\prime}} \bar{p}_{h, h_{0}} r_{h_{0}, h_{1}} \bar{r}_{h_{1}, h^{\prime}} \\
& =\sum_{h_{0} ; h \leq h_{0}<h^{\prime}} \bar{p}_{h, h_{0}} \sum_{h_{1} ; h_{0} \leq h_{1} \leq h^{\prime}} \bar{r}_{h_{0}, h_{1}} r_{h_{1}, h^{\prime}} \\
& =\sum_{h_{0} ; h \leq h_{0}<h^{\prime}} \bar{p}_{h, h_{0}} \delta_{h_{0}, h^{\prime}}=0 .
\end{aligned}
$$

Now we claim that since $\bar{q}+q=0$, there is a unique $q^{\prime} \in v \mathbb{Z}^{\pi}[v]$ such that $q^{\prime}-\overline{q^{\prime}}=0$.

Indeed, we can write $q=\sum_{m, n \in \mathbb{N}} a_{n} v^{n}$ with $a_{n} \in \mathbb{Z}^{\pi}$ for $n \in \mathbb{N}$. Then since $q+\bar{q}=0$, we see that $a_{n}=-\pi^{n} a_{-n}$ for all $m, n \in \mathbb{N}$. In particular, $a_{0}=0$ and $a_{n}=0$ if and only if $a_{-n}=0$ for $n \in \mathbb{Z}$. Then taking $q^{\prime}=\sum_{n \in \mathbb{N}} a_{n} v^{n}$, we see that $q=q^{\prime}-\overline{q^{\prime}}$ and $q^{\prime} \in v \mathbb{Z}^{\pi}[v]$. In particular, we can set $p_{h, h^{\prime}}=q^{\prime}$, and then

$$
p_{h, h^{\prime}}=q+\overline{q^{\prime}}=\sum_{h^{\prime \prime} ; h \leq h^{\prime \prime}<h^{\prime}} \bar{p}_{h, h^{\prime \prime}} r_{h^{\prime \prime}, h^{\prime}}+\overline{p_{h, h^{\prime}}}=\sum_{h^{\prime \prime} ; h \leq h^{\prime \prime} \leq h^{\prime}} \bar{p}_{h, h^{\prime \prime}} r_{h^{\prime \prime}, h^{\prime}}
$$

Remark 4.4. For $R$ a commutative ring, we can define a bar-involution on $A=R[x, y]$ by $\overline{f(x, y)}=f(y, x)$. This involutions descends to a bar-involution on $A_{r}=R[x, y] /(x y-$ $r) R[x, y] \cong R\left[x, r x^{-1}\right]$ for any $r \in R$. Then the assertions of the lemma apply with $\mathbb{A}$ replaced by $A$ or $A_{r}, v$ replaced with $x$, and with $\mathbb{Z}^{\pi}[v]$ replaced everywhere by $R[x]$. In particular, Lemma 4.3 (respectively, [L93, §24.2]) is a special case for $R=\mathbb{Z}^{\pi}$ and $r=\pi$ (respectively, $R=\mathbb{Z}$ and $r=1$ ).

4.3. The canonical basis of $N\left(\lambda, \lambda^{\prime}\right)$. Let $\lambda, \lambda^{\prime} \in X^{+}$. We shall consider the following partial order on the set $\mathcal{B} \times_{\pi} \mathcal{B}$ : we say that $\left(b_{1}, b_{1}^{\prime}\right) \leq\left(b_{2}, b_{2}^{\prime}\right)$ if

$$
\mathrm{ht}\left|b_{1}\right|-\mathrm{ht}\left|b_{1}^{\prime}\right|=\mathrm{ht}\left|b_{2}\right|-\mathrm{ht}\left|b_{2}^{\prime}\right|
$$

and if we have either

$$
\mathrm{ht}\left|b_{1}\right|<\mathrm{ht}\left|b_{2}\right| \text { and ht }\left|b_{1}^{\prime}\right|<\mathrm{ht}\left|b_{2}^{\prime}\right| \text {, }
$$

or

$$
\left(b_{1}, b_{2}\right) \sim\left(b_{1}^{\prime}, b_{2}^{\prime}\right) .
$$

Note that, in particular, $\left(b_{1}, b_{1}^{\prime}\right)$ and $\left(b_{1}, \pi b_{1}^{\prime}\right)$ are not comparable under $\leq$. For given $\lambda, \lambda^{\prime} \in X^{+}$, this induces a partial order on the set $\mathcal{B}(\lambda) \times_{\pi} \mathcal{B}\left(\lambda^{\prime}\right)$.

Then from the definition, we have that for all $\left(b_{1}, b_{1}^{\prime}\right) \in B(\lambda) \times{ }_{\pi} B\left(\lambda^{\prime}\right)$,

$$
\Psi\left(b_{1}^{-} \eta_{\lambda} \otimes b_{1}^{\prime+} \xi_{-\lambda^{\prime}}\right)=\sum_{\left(b_{2}, b_{2}^{\prime}\right) \in B(\lambda) \times_{\pi} B\left(\lambda^{\prime}\right)} r_{b_{1}, b_{1}^{\prime} ; b_{2}, b_{2}^{\prime}} b_{2}^{-} \eta_{\lambda} \otimes b_{2}^{\prime+} \xi_{-\lambda^{\prime}},
$$

where $r_{b_{1}, b_{1}^{\prime} ; b_{2}, b_{2}^{\prime}} \in \mathbb{Z}\left[v^{ \pm 1}\right]$ and $r_{b_{1}, b_{1}^{\prime} ; b_{2}, b_{2}^{\prime}}=0$ unless $\left(b_{1}, b_{1}^{\prime}\right) \geq\left(b_{2}, b_{2}^{\prime}\right)$; in particular, the sum is finite. 
Moreover, we note that $r_{b_{1}, b_{1}^{\prime} ; b_{1}, b_{1}^{\prime}}=1$, and from $\Psi^{2}=1$ we see that

$$
\sum_{\left(b_{1}, b_{1}^{\prime}\right) ;\left(b_{2}, b_{2}^{\prime}\right) ;\left(b_{3}, b_{3}^{\prime}\right) \in B(\lambda) \times_{\pi} B\left(\lambda^{\prime}\right)} \bar{r}_{b_{1}, b_{1}^{\prime} ; b_{2}, b_{2}^{\prime}} r_{b_{2}, b_{2}^{\prime} ; b_{3}, b_{3}^{\prime}}=\delta_{\left(b_{1}, b_{1}^{\prime}\right) ;\left(b_{3}, b_{3}^{\prime}\right)} .
$$

Then $H=B(\lambda) \times_{\pi} B\left(\lambda^{\prime}\right)$ and $r_{b_{1}, b_{1}^{\prime} ; b_{2}, b_{2}^{\prime}}$ satisfy the assumptions of Lemma 4.3, so there exist elements $p_{b_{1}, b_{1}^{\prime} ; b_{2}, b_{2}^{\prime}} \in \mathbb{Z}^{\pi}[v]$ such that

$$
\begin{gathered}
p_{b_{1}, b_{1}^{\prime} ; b_{1}, b_{1}^{\prime}}=1, \\
p_{b_{1}, b_{1}^{\prime} ; b_{2}, b_{2}^{\prime}}=0 \text { unless }\left(b_{2}, b_{2}^{\prime}\right) \leq\left(b_{1}, b_{1}^{\prime}\right), \\
p_{b_{1}, b_{1}^{\prime} ; b_{2}, b_{2}^{\prime}} \in v \mathbb{Z}^{\pi}[v] \text { for }\left(b_{2}, b_{2}^{\prime}\right)<\left(b_{1}, b_{1}^{\prime}\right), \\
p_{b_{1}, b_{1}^{\prime} ; b_{3}, b_{3}^{\prime}}=\sum_{\left(b_{2}, b_{2}^{\prime}\right) \in B(\lambda) \times_{\pi} B\left(\lambda^{\prime}\right)} \bar{p}_{b_{1}, b_{1}^{\prime} ; b_{2}, b_{2}^{\prime}} r_{b_{2}, b_{2}^{\prime} ; b_{3}, b_{3}^{\prime}} .
\end{gathered}
$$

Now recall from Section 3.5 that $\mathcal{L}\left(\right.$ resp. $\mathbb{A} \mathcal{L}$ ) is the $\mathbb{Z}^{\pi}[v]$-lattice (resp. $\mathbb{A}$-lattice) with basis $\mathcal{B}\left(\lambda, \lambda^{\prime}\right)=\left\{b^{+} \xi_{-\lambda} \otimes b^{-} \eta_{\lambda^{\prime}}: b \times b^{\prime} \in B(\lambda) \times_{\pi} B\left(\lambda^{\prime}\right)\right\}$.

Theorem 4.5. (1) For any $\left(b, b^{\prime}\right) \in B(\lambda) \times_{\pi} B\left(\lambda^{\prime}\right)$, there is a unique element $\left(b \diamond b^{\prime}\right)_{\lambda, \lambda^{\prime}}$ of the $\mathbf{U}$-module $N\left(\lambda, \lambda^{\prime}\right)$ such that

$$
\Psi\left(\left(b \diamond b^{\prime}\right)_{\lambda, \lambda^{\prime}}\right)=\left(b \diamond b^{\prime}\right)_{\lambda, \lambda^{\prime}} \text { and }\left(b \diamond b^{\prime}\right)_{\lambda, \lambda^{\prime}}-b^{-} \eta_{-\lambda} \otimes b^{\prime+} \xi_{-\lambda^{\prime}} \in v \mathcal{L} .
$$

(2) We have $\left(\pi b \diamond b^{\prime}\right)_{\lambda, \lambda^{\prime}}=\left(b \diamond \pi b^{\prime}\right)_{\lambda, \lambda^{\prime}}=\pi\left(b \diamond b^{\prime}\right)_{\lambda, \lambda^{\prime}}$

(3) $\left(b \diamond b^{\prime}\right)_{\lambda, \lambda^{\prime}}$ is equal to $b^{-} \eta_{\lambda} \otimes b^{\prime+} \xi_{-\lambda^{\prime}}$ plus a $v \mathbb{Z}^{\pi}[v]$-linear combination of elements $b_{1}^{-} \eta_{\lambda} \otimes b_{1}^{\prime+} \xi_{-\lambda^{\prime}}$ with $\left(b_{1}, b_{1}^{\prime}\right)<\left(b, b^{\prime}\right)$.

(4) The elements $\left(b \diamond b^{\prime}\right)_{\lambda, \lambda^{\prime}}$ form a $\pi$-basis of $\mathcal{L}, \mathbb{A} \mathcal{L}$, and $N\left(\lambda, \lambda^{\prime}\right)$.

(5) The natural homomorphism $\mathcal{L} \cap \Psi(\mathcal{L}) \rightarrow \mathcal{L} / v \mathcal{L}$ is an isomorphism.

Proof. By the definition of $p_{b, b^{\prime} ; b_{1}, b_{1}^{\prime}}$, we see that

$$
\left(b \diamond b^{\prime}\right)_{\lambda, \lambda^{\prime}}=\sum_{\left(b_{1}, b_{1}^{\prime}\right) \leq\left(b, b^{\prime}\right)} p_{b, b^{\prime} ; b_{1}, b_{1}^{\prime}} b_{1}^{-} \eta_{\lambda} \otimes b_{1}^{\prime+} \xi_{-\lambda^{\prime}}
$$

satisfies the requirements of (1) proving existence, and the same considerations prove (3). (4) is immediate from the fact that the transition matrix from the $\mathbb{Q}(v)$-basis $b_{1}^{-} \eta_{\lambda} \otimes$ $b_{1}^{\prime+} \xi_{-\lambda^{\prime}}$ is unitriangular with entries in $\mathbb{Z}[v]$. (5) follows from (4) and the observation that $\left(b \diamond b^{\prime}\right)_{\lambda, \lambda^{\prime}} \in \mathcal{L} \cap \Psi(\mathcal{L})$, and so the map sends $\left(b \diamond b^{\prime}\right)_{\lambda, \lambda^{\prime}}$ to the basis element $b^{-} \eta_{\lambda} \otimes b^{\prime+} \xi_{-\lambda^{\prime}}$ of $\mathcal{L} / v \mathcal{L}$, which also implies uniqueness. Finally, uniqueness implies (2).

We call the elements $\left(b \diamond b^{\prime}\right)_{\lambda, \lambda^{\prime}}$ the canonical basis of $N\left(\lambda, \lambda^{\prime}\right)$.

Remark 4.6. We may repeat verbatim 4.1 with respect to $\Theta_{4}$ to obtain a bar-involution $\Psi_{4}$ on $N_{4}\left(\lambda, \lambda^{\prime}\right)$. Then we see that the results of this section (and in particular Theorem 4.5) may be restated with $\Psi, N\left(\lambda, \lambda^{\prime}\right)$ replaced by $\Psi_{4}, N_{4}\left(\lambda, \lambda^{\prime}\right)$. When we need to distinguish them, we denote the canonical basis of $N_{s}\left(\lambda, \lambda^{\prime}\right)$ by $\left(b \diamond_{s} b^{\prime}\right)_{\lambda, \lambda^{\prime}}$.

4.4. Cancellation and stability. Our goal in this subsection is to exhibit maps between modules in $\mathcal{F}$ which are compatible with canonical bases. These maps will correspond to a form of cancellation on the pairs $\left(\lambda, \lambda^{\prime}\right) \in X^{+} \times X^{+}$; namely, the cancellation

$$
\left(\lambda+\lambda^{\prime \prime}, \lambda^{\prime \prime}+\lambda^{\prime}\right) \mapsto\left(\lambda, \lambda^{\prime}\right)
$$

can be realized as a $\mathbf{U}$-module homomorphism $N\left(\lambda+\lambda^{\prime \prime}, \lambda^{\prime \prime}+\lambda^{\prime}\right) \rightarrow N\left(\lambda, \lambda^{\prime}\right)$.

Proposition 4.7. Let $\lambda, \lambda^{\prime} \in X^{+}$. Write $\eta=\eta_{\lambda}, \eta^{\prime}=\eta_{\lambda^{\prime}}$, and $\eta^{\prime \prime}=\eta_{\lambda+\lambda^{\prime}}$.

(1) There is a unique homomorphism of $\mathbf{U}$-modules $\chi: V\left(\lambda+\lambda^{\prime}\right) \rightarrow V(\lambda) \otimes V\left(\lambda^{\prime}\right)$ such that $\chi\left(\eta^{\prime \prime}\right)=\eta \otimes \eta^{\prime}$. 
(2) Let $b \in B\left(\lambda+\lambda^{\prime}\right)$. We have $\chi\left(b^{-} \eta^{\prime \prime}\right)=\sum_{b_{1}, b_{2}} f\left(b ; b_{1}, b_{2}\right) b_{1}^{-} \eta \otimes b_{2}^{-} \eta^{\prime}$ where the sum is over $\left(b_{1}, b_{2}\right) \in B(\lambda) \times_{\pi} B\left(\lambda^{\prime}\right)$ and $f\left(b ; b_{1}, b_{2}\right) \in \mathbb{Z}[v]$.

(3) If $b^{-} \eta^{\prime} \neq 0$, then $f(b ; b, 1)=1$ and $f\left(b ; b_{1}, 1\right)=0$ for any $b_{1} \neq b$. If $b^{-} \eta^{\prime}=0$, then $\left.f\left(b ; b_{1}, 1\right)\right)=0$ for any $b_{1}$.

Proof. The map $\chi$ is the same as the map $\Phi^{\prime}\left(\lambda, \lambda^{\prime}\right)$ as defined in [CHW2] (with reversed conventions on $\Delta, \Delta^{\prime} ; \otimes, \otimes^{\prime}$ ), proving (1). A minor variation on the proof of [CHW2, Lemma 5.7] shows that $\Phi^{\prime}\left(\lambda, \lambda^{\prime}\right)$ preserves the crystal lattice. Combining this with the fact that $\mathcal{B} \subset{ }_{\mathbb{A}} \mathbf{f}$, we see that $f\left(b ; b_{1}, b_{2}\right) \in \mathbb{Z}^{\pi}[v] ;$ moreover, up to identifying $\pi\left(b_{1}^{-} \eta \otimes b_{2}^{-} \eta^{\prime}\right)$ with $\left(\pi b_{1}^{-}\right) \eta \otimes b_{2}^{-} \eta^{\prime}$, we may assume that $f\left(b ; b_{1}, b_{2}\right) \in \mathbb{Z}[v]$. (3) is immediate from the definition of the coproduct.

Remark 4.8. In [CHW2, there is another map

$$
\Phi\left(\lambda, \lambda^{\prime}\right): V\left(\lambda+\lambda^{\prime}\right) \rightarrow V(\lambda) \otimes_{4} V\left(\lambda^{\prime}\right)
$$

and there is a version of the above proposition where we replace $\chi, \otimes$ with $\Phi, \otimes_{4}$ everywhere.

Proposition 4.9. Let $\lambda, \lambda^{\prime} \in X^{+}$. Write $\xi=\xi_{-\lambda}, \xi^{\prime}=\xi_{-\lambda^{\prime}}$, and $\xi^{\prime \prime}=\xi_{-\lambda-\lambda^{\prime}}$.

(1) There is a unique homomorphism of $\mathbf{U}$-modules $\chi_{4}:{ }^{\omega} V\left(\lambda+\lambda^{\prime}\right) \rightarrow{ }^{\omega} V\left(\lambda^{\prime}\right) \otimes{ }^{\omega} V(\lambda)$ such that $\chi_{4}\left(\xi^{\prime \prime}\right)=\xi^{\prime} \otimes \xi$.

(2) Let $b \in \mathcal{B}\left(\lambda+\lambda^{\prime}\right)_{\nu}$. We have

$$
\chi_{4}\left(b^{+} \xi^{\prime \prime}\right)=\sum_{b_{1}, b_{2}} \pi^{p\left(b_{1}\right) p\left(b_{2}\right)} f\left(b ; b_{1}, b_{2}\right) b_{2}^{+} \xi^{\prime} \otimes b_{1}^{+} \xi
$$

where the sum is over $\left(b_{1}, b_{2}\right) \in B(\lambda) \times_{\pi} B\left(\lambda^{\prime}\right)$ and $f\left(b ; b_{1}, b_{2}\right) \in \mathbb{Z}^{\pi}[v]$.

(3) If $b^{+} \xi^{\prime} \neq 0$, then $f(b ; b, 1)=1$ and $f\left(b ; b_{1}, 1\right)=0$ for any $b_{1} \neq b$. If $b^{+} \xi^{\prime}=0$, then $\left.f\left(b ; b_{1}, 1\right)\right)=0$ for any $b_{1}$.

Proof. By Proposition 4.7 and Remark 4.8, there is a homomorphism

$$
\Phi: V\left(\lambda+\lambda^{\prime}\right) \rightarrow V(\lambda) \otimes_{4} V\left(\lambda^{\prime}\right) .
$$

Then $\Phi$ can also be viewed as a homomorphism ${ }^{\omega} V\left(\lambda+\lambda^{\prime}\right) \rightarrow{ }^{\omega}\left(V(\lambda) \otimes_{4} V\left(\lambda^{\prime}\right)\right)$. By Lemma 3.7, we have an isomorphism

$$
{ }^{\omega}\left(V(\lambda) \otimes_{4} V\left(\lambda^{\prime}\right)\right) \rightarrow{ }^{\omega} V\left(\lambda^{\prime}\right) \otimes{ }^{\omega} V(\lambda) \quad y \otimes z \mapsto \pi^{p(y) p(z)} z \otimes y .
$$

Then taking $\chi^{\prime}$ to be the composition of these homomorphisms proves (1). The remaining properties follow by the definitions.

Proposition 4.10. Let $\eta=\eta_{\lambda}$ and $\xi=\xi_{-\lambda}$.

(1) There is a unique homomorphism of $\mathbf{U}$-modules $\delta_{\lambda}: N(\lambda, \lambda) \rightarrow \mathbb{Q}(v)^{\pi}$, where $\mathbb{Q}(v)$ is a $\mathbf{U}$-module under the counit map, such that $\delta_{\lambda}(\xi \otimes \eta)=1$.

(2) Let $b, b^{\prime} \in B(\lambda)$. Then $\delta_{\lambda}\left(b^{+} \xi \otimes b^{\prime-} \eta\right)=1$ if $b=b^{\prime}=1$ and is in $v \mathbb{Z}^{\pi}[v]$ otherwise.

Proof. For such a map to exist, we would need

$$
\Delta\left(E_{i}\right)(x \otimes y)=E_{i} x \otimes \tilde{K}_{i} y+\pi_{i}^{p(x)} x \otimes F_{i} y \in \operatorname{ker}\left(\delta_{\lambda}\right) \text { for all } x \otimes y \in N(\lambda, \lambda) ;
$$

$\Delta\left(F_{i}\right)(x \otimes y)=F_{i} x \otimes y+\pi_{i}^{p(x)} \tilde{J}_{i} \tilde{K}_{i} x \otimes \pi_{i} \tilde{J}_{i} E_{i} y \in \operatorname{ker}\left(\delta_{\lambda}\right)$ for all $x \otimes y \in N(\lambda, \lambda) ;$

$\Delta\left(K_{\mu}-1\right)(x \otimes y)=K_{\mu} x \otimes K_{-\mu} y-x \otimes y \in \operatorname{ker}\left(\delta_{\lambda}\right)$ for all $x \otimes y \in N(\lambda, \lambda)$;

$$
\Delta\left(J_{\mu}-1\right)(x \otimes y)=J_{\mu} x \otimes J_{\mu} y-x \otimes y \in \operatorname{ker}\left(\delta_{\lambda}\right) \text { for all } x \otimes y \in N(\lambda, \lambda) ;
$$


The following statement is equivalent to (1). There is a unique bilinear pairing [,] : $V(\lambda) \times V(\lambda) \rightarrow \mathbb{Q}(v)^{\pi}$ satisfying $[\eta, \eta]=1$,

$$
\begin{gathered}
{\left[E_{i} x, \tilde{K}_{i} y\right]=-\pi_{i}^{p(x)}\left[x, F_{i} y\right], \quad\left[F_{i} x, y\right]=-\pi_{i}^{p(x)}\left[\tilde{J}_{i} \tilde{K}_{i} x, \pi_{i} \tilde{J}_{i} E_{i} y\right]} \\
{\left[K_{\mu} x, y\right]=\left[x, K_{\mu} y\right], \quad\left[J_{\mu} x, y\right]=\left[x, J_{\mu} y\right] .}
\end{gathered}
$$

We may rewrite the conditions as

$$
\begin{array}{cc}
{\left[E_{i} x, y\right]=-\pi_{i}^{p(x)}\left[x, F_{i} \tilde{K}_{-i} y\right], \quad\left[F_{i} x, y\right]=-\pi_{i}^{p(x)}\left[x, \pi_{i} \tilde{K}_{i} F_{i} y\right]} \\
{\left[K_{\mu} x, y\right]=\left[x, K_{\mu} y\right], \quad\left[J_{\mu} x, y\right]=\left[x, J_{\mu} y\right] .}
\end{array}
$$

Let a : $\mathbf{U} \rightarrow \mathbf{U}$ be the $\mathbb{Q}(v)^{\pi}$-linear map defined by

$$
\begin{gathered}
\boldsymbol{\beta}\left(E_{i}\right)=-F_{i} \tilde{K}_{-i}, \quad \boldsymbol{\beta}\left(F_{i}\right)=-\pi_{i} \tilde{K}_{i} E_{i}, \quad \boldsymbol{\beta}\left(K_{\mu}\right)=K_{\mu}, \quad \boldsymbol{\beta}\left(J_{\mu}\right)=J_{\mu}, \\
\boldsymbol{\beta}(x y)=\pi^{p(x) p(y)} \boldsymbol{\beta}(y) \boldsymbol{\beta}(x) .
\end{gathered}
$$

To see this is a well-defined map, we note that $\mathbf{p}=\omega S$ where $S$ is the antipode of $\Delta$; that is, the $\mathbb{Q}(v)^{\pi}$-linear map satisfying

$$
\begin{gathered}
S\left(E_{i}\right)=-E_{i} \tilde{K}_{i}, \quad S\left(F_{i}\right)=-\tilde{J}_{i} \tilde{K}_{-i} F_{i}, \quad S\left(K_{\mu}\right)=K_{-\mu}, \quad S\left(J_{\mu}\right)=J_{\mu}, \\
S(x y)=\pi^{p(x) p(y)} S(y) S(x) .
\end{gathered}
$$

(Note that this is not the antipode defined in [CHW1, §2.4], as that map corresponds to the coproduct $\Delta_{1}$.)

Then we see that (1) is equivalent to proving the existence of a unique bilinear pairing $[]:, V(\lambda) \times V(\lambda) \rightarrow \mathbb{Q}(v)^{\pi}$ satisfying $[\eta, \eta]=1$ and $[u x, y]=\pi^{p(x) p(u)}[x, \boldsymbol{\alpha}(u) y]$. This is proven using a standard argument: in brief, the restricted dual $V(\lambda)^{*}$ has a $\mathbf{U}$-action $(u f)(x)=\pi^{p(u) p(f)} f(\boldsymbol{\beta}(x) u)$ under which we have an isomorphism $V(\lambda)^{*} \cong V(\lambda)$, and $[\cdot, \cdot]$ is the natural pairing.

Let $(\cdot, \cdot)$ be the polarization on $V(\lambda)$. We show by induction on $h t \nu \geq 0$ that

$$
[x, y]=(-1)^{\mathrm{ht} \nu} \pi^{\mathbf{p}(\nu)} \pi_{\nu} v_{\nu}(x, y)
$$

for $x, y \in V(\lambda)_{\lambda-\nu}$, where here we set $\mathbf{p}\left(\sum_{t} i_{t}\right)=\sum_{s<t} p\left(i_{s}\right) p\left(i_{t}\right)$.

This is obvious for $\nu=0$. Assume that ht $\nu \geq 1$. Then we can assume that $x=F_{i} x^{\prime}$ for some $i$ such that $\nu_{i}>0$. Then by induction, we compute

$$
\begin{aligned}
{[x, y] } & =\left[F_{i} x^{\prime}, y\right]=-\pi_{i}^{p\left(x^{\prime}\right)}\left[x^{\prime}, \pi_{i} \tilde{K}_{i} E_{i} y\right]=-\pi_{i}^{p(\nu-i)} \pi_{i}\left[x^{\prime}, \tilde{K}_{i} E_{i} y\right] \\
& =-\pi_{i}^{p(\nu-i)} \pi_{i}(-1)^{\mathrm{ht}(\nu-i)} \pi_{i}^{\mathbf{p}(\nu-i)} \pi_{\nu-i} v_{\nu-i}\left(x^{\prime}, \tilde{K}_{i} E_{i} y\right) \\
& =(-1)^{\mathrm{ht} \nu} \pi^{\mathbf{p}(\nu)} \pi_{\nu} v_{\nu}\left(F_{i} x^{\prime}, y\right)=(-1)^{\mathrm{ht} \nu} \pi^{\mathbf{p}(\nu)} \pi_{\nu} v_{\nu}(x, y) .
\end{aligned}
$$

This proves that (4.1) holds. Now recall from Lemma $2.14(2)$ that $\left(b \eta, b^{\prime} \eta\right) \in \mathbb{Z}^{\pi}[v]$ for any $b, b^{\prime} \in \mathcal{B}$. Combining this with (4.1), we see that (2) follows.

We have demonstrated the existence of the maps $\chi, \chi_{4}$ Let $\lambda, \lambda^{\prime}, \lambda^{\prime \prime} \in X^{+}$. We define a U-module homomorphism

$$
t: N\left(\lambda+\lambda^{\prime}, \lambda^{\prime}+\lambda^{\prime \prime}\right) \rightarrow N\left(\lambda, \lambda^{\prime \prime}\right)
$$

defined as the composition

$$
t=\left(1 \otimes \delta_{\lambda} \otimes 1\right) \circ\left(\chi \otimes \chi^{\prime}\right) .
$$

Lemma 4.11. We have $\Psi t=t \Psi$. 
Proof. We write $\eta=\eta_{\lambda+\lambda^{\prime}}$ and $\xi=\xi_{-\lambda^{\prime}-\lambda^{\prime \prime}}$. Since any element of $N\left(\lambda+\lambda^{\prime}, \lambda^{\prime} \otimes \lambda^{\prime \prime}\right)$ is of the form $u(\eta \otimes \xi)$, it is enough to check that

$$
t \Theta(\overline{u(\eta \otimes \xi)})=\Theta(\overline{t(u(\eta \otimes \xi))}) .
$$

Well, on one hand

$$
t \Theta(\overline{u(\eta \otimes \xi)})=t(u \Theta(\eta \otimes \xi))=u t((\eta \otimes \xi))=u\left(\eta_{\lambda} \otimes \xi_{-\lambda^{\prime \prime}}\right) .
$$

On the other hand,

$$
\Theta(\overline{t(u(\eta \otimes \xi))})=\Theta(\overline{u t(\eta \otimes \xi)})=u \Theta\left(\eta_{\lambda} \otimes \xi_{-\lambda^{\prime \prime}}\right)=u\left(\eta_{\lambda} \otimes \xi_{-\lambda^{\prime \prime}}\right) .
$$

The lemma is proved.

Lemma 4.12. (1) Let $b \in \mathcal{B}(\lambda)$ and $b^{\prime \prime} \in \mathcal{B}\left(\lambda^{\prime \prime}\right)$. Then

$$
t\left(b^{-} \eta_{\lambda+\lambda^{\prime}} \otimes b^{\prime \prime+} \xi_{-\lambda^{\prime}-\lambda^{\prime \prime}}\right)=b^{-} \eta_{\lambda} \otimes b^{\prime \prime+} \xi_{-\lambda^{\prime \prime}} \bmod v \mathcal{L}\left(\lambda, \lambda^{\prime \prime}\right) .
$$

In particular, $t\left(\left(b \diamond b^{\prime}\right)_{\lambda+\lambda^{\prime}, \lambda^{\prime}+\lambda^{\prime \prime}}\right)=\left(b \diamond b^{\prime}\right)_{\lambda, \lambda^{\prime \prime}} \bmod v \mathcal{L}\left(\lambda, \lambda^{\prime \prime}\right)$.

(2) Let $b \in \mathcal{B}\left(\lambda+\lambda^{\prime}\right)$ and $b^{\prime \prime} \in \mathcal{B}\left(\lambda^{\prime}+\lambda^{\prime \prime}\right)$. Assume that either $b \notin \mathcal{B}(\lambda)$ or $b^{\prime \prime} \notin \mathcal{B}\left(\lambda^{\prime \prime}\right)$. Then

$$
t\left(b^{-} \eta_{\lambda+\lambda^{\prime}} \otimes b^{\prime \prime+} \xi_{-\lambda^{\prime}-\lambda^{\prime \prime}}\right)=0 \bmod v \mathcal{L}\left(\lambda, \lambda^{\prime \prime}\right) .
$$

In particular, $t\left(\left(b \diamond b^{\prime}\right)_{\lambda+\lambda^{\prime}, \lambda^{\prime}+\lambda^{\prime \prime}}\right)=0 \bmod v \mathcal{L}\left(\lambda, \lambda^{\prime \prime}\right)$.

(3) $t$ is surjective.

Proof. Parts (1) and (2) follow from Propositions 4.744.10 and the definition of $t$. In particular, note that $t\left(\eta_{\lambda+\lambda^{\prime}} \otimes \xi_{-\lambda^{\prime}-\lambda^{\prime \prime}}\right)=\eta_{\lambda} \otimes \xi_{-\lambda^{\prime \prime}}$, which generates $N\left(\lambda, \lambda^{\prime \prime}\right)$.

Proposition 4.13. (1) Let $b \in \mathcal{B}(\lambda)$ and $b^{\prime \prime} \in \mathcal{B}\left(\lambda^{\prime \prime}\right)$. Then

$$
t\left(\left(b \diamond b^{\prime}\right)_{\lambda+\lambda^{\prime}, \lambda^{\prime}+\lambda^{\prime \prime}}\right)=\left(b \diamond b^{\prime}\right)_{\lambda, \lambda^{\prime \prime}}
$$

(2) Let $b \in \mathcal{B}\left(\lambda+\lambda^{\prime}\right)$ and $b^{\prime \prime} \in \mathcal{B}\left(\lambda^{\prime}+\lambda^{\prime \prime}\right)$. Assume that $b \notin \mathcal{B}(\lambda)$ or $b^{\prime \prime} \notin \mathcal{B}\left(\lambda^{\prime \prime}\right)$. Then

$$
t\left(\left(b \diamond b^{\prime}\right)_{\lambda+\lambda^{\prime}, \lambda^{\prime}+\lambda^{\prime \prime}}\right)=0 .
$$

Proof. The differences of the two sides of the claimed equalities in (1) and (2) lie in $v \mathcal{L}\left(\lambda, \lambda^{\prime \prime}\right)$ and are fixed by $\Psi$, hence the difference is zero.

4.5. The canonical basis of $\dot{\mathbf{U}}$. We are now in a position to produce a canonical basis for $\dot{\mathbf{U}}$ which descends to the canonical bases for modules in $\mathcal{F}$.

Theorem 4.14. Let $\zeta \in X$ and $\left(b, b^{\prime \prime}\right) \in \mathcal{B} \times_{\pi} \mathcal{B}$.

(1) There is a unique element $u=b \diamond_{\zeta} b^{\prime \prime} \in{ }_{\mathbb{A}} \dot{\mathbf{U}}$ such that

$$
\Delta(u)\left(\eta_{\lambda} \otimes \xi_{-\lambda^{\prime \prime}}\right)=\left(b \diamond b^{\prime \prime}\right)_{\lambda, \lambda^{\prime \prime}}
$$

for any $\lambda, \lambda^{\prime \prime} \in X^{+}$such that $b \in B(\lambda), b^{\prime \prime} \in B\left(\lambda^{\prime \prime}\right)$, and $\zeta=\lambda-\lambda^{\prime \prime}$.

(2) If $\lambda, \lambda^{\prime \prime} \in X^{+}$are such that $\lambda-\lambda^{\prime \prime}=\zeta$ and either $b \notin \mathcal{B}(\lambda)$ or $b^{\prime \prime} \notin \mathcal{B}\left(\lambda^{\prime \prime}\right)$, then

$$
\Delta\left(b \diamond b^{\prime \prime}\right)\left(\eta_{\lambda} \otimes \xi_{-\lambda^{\prime \prime}}\right)=0 .
$$

(3) $\overline{b \diamond \zeta b^{\prime \prime}}=b \diamond_{\zeta} b^{\prime \prime}$

(4) The elements $b \diamond_{\zeta} b^{\prime \prime}$, for various $\zeta, b, b^{\prime \prime}$ as above, form $a \mathbb{Q}(v)^{\pi}$-basis of $\mathbf{U}$ and $a$ $\mathbb{A}$-basis of $\dot{\mathbb{U}}$. 
Proof. First recall that, throughout the paper, we assume the root datum is $Y$-regular. Then we can find $\lambda, \lambda^{\prime \prime} \in X^{+}$such that $b \in B(\lambda), b^{\prime \prime} \in B\left(\lambda^{\prime \prime}\right)$, and $\lambda-\lambda^{\prime \prime}=\zeta$.

For any integers $N_{1}, N_{2}$, let $P\left(N_{1}, N_{2}\right)$ be the $\mathbb{A}$-submodule of ${ }_{\mathbb{A}} \dot{\mathbf{U}}$ spanned by the elements $b_{1}^{-} b_{2}^{+} 1_{\zeta}$ where $b_{1}$ and $b_{2}$ run through the elements of $\mathcal{B}$ such that ht $\left|b_{1}\right| \leq N_{1}$, $\mathrm{ht}\left|b_{2}\right| \leq N_{2}$, and $\left|b_{1}\right|-\left|b_{2}\right|=|b|-\left|b^{\prime \prime}\right|$.

Recall that any element of $N\left(\lambda, \lambda^{\prime \prime}\right)$ of the form $\beta^{-} \eta_{\lambda} \otimes \beta^{\prime+} \xi_{-\lambda}$ with $\beta, \beta^{\prime} \in \mathcal{B}$ is equal to $u_{1}\left(\eta_{\lambda} \otimes \xi_{-\lambda}\right)$ for some $u_{1} \in P\left(\mathrm{ht}|\beta|\right.$, ht $\left.\left|\beta^{\prime}\right|\right)$; moreover, $u_{1}$ can be taken to be equal to $\beta^{-} \beta^{\prime+} 1_{\zeta}$ plus an element in $P\left(\right.$ ht $|\beta|-1$, ht $\left.\left|\beta^{\prime}\right|-1\right)$. In particular, we see that $\left(b \diamond b^{\prime \prime}\right)_{\lambda, \lambda^{\prime \prime}}$ is of the form $u\left(\eta_{\lambda} \otimes \xi_{-\lambda^{\prime \prime}}\right)$ for some $u \in P\left(\mathrm{ht}|b|, \mathrm{ht}\left|b^{\prime \prime}\right|\right)$; moreover, $u$ can be taken to be equal to $b^{-} b^{\prime \prime+} 1_{\zeta}$ plus an element in $P\left(\mathrm{ht}|b|-1\right.$, ht $\left.\left|b^{\prime \prime}\right|-1\right)$.

Assume that $u$ is such an element and $u^{\prime}$ is another such element. Then $\left(u-u^{\prime}\right)\left(\eta_{\lambda} \otimes\right.$ $\left.\xi_{-\lambda^{\prime \prime}}\right)=0$, and so by Proposition 3.9

$$
\left(u-u^{\prime}\right) \in \sum_{i, n>\langle i, \lambda\rangle} \dot{\mathbb{U}} F_{i}^{(n)} 1_{\zeta}+\sum_{i, n>\left\langle i, \lambda^{\prime \prime}\right\rangle} \dot{\mathbb{U}} E_{i}^{(n)} 1_{\zeta} .
$$

However, since $u-u^{\prime} \in P\left(\mathrm{ht}|b|\right.$, ht $\left.\left|b^{\prime \prime}\right|\right)$, we must have $u-u^{\prime}=0$ if $\langle i, \lambda\rangle>\mathrm{ht}|b|$ and $\left\langle i, \lambda^{\prime \prime}\right\rangle>\mathrm{ht}\left|b^{\prime \prime}\right|$ for all $i \in I$. For such $\lambda, \lambda^{\prime \prime}$ the element $u$ is uniquely determined, and we denote it by $u_{\lambda, \lambda^{\prime \prime}}$.

Assume now that $\lambda, \lambda^{\prime \prime} \in X^{+}$satisfy $b \in \mathcal{B}(\lambda), b^{\prime \prime} \in \mathcal{B}\left(\lambda^{\prime \prime}\right)$, and $\lambda-\lambda^{\prime \prime}=\zeta$. Let $\lambda^{\prime} \in X^{+}$such that $\lambda>>0$ so that $u^{\prime}=u_{\lambda+\lambda^{\prime}, \lambda^{\prime}+\lambda^{\prime \prime}}$ is defined. Then

$$
u^{\prime}\left(\eta_{\lambda} \otimes \xi_{-\lambda^{\prime \prime}}\right)=u^{\prime} t\left(\eta_{\lambda+\lambda^{\prime}} \otimes \xi_{-\lambda^{\prime}-\lambda^{\prime \prime}}\right)=t\left(\left(b \diamond b^{\prime \prime}\right)_{\lambda+\lambda^{\prime}, \lambda^{\prime}+\lambda^{\prime \prime}}\right)=\left(b \diamond b^{\prime \prime}\right)_{\lambda, \lambda^{\prime \prime}} .
$$

Then $u_{\lambda, \lambda^{\prime \prime}}$ is independent of $\lambda, \lambda^{\prime \prime}$ if it is defined, so we may denote it as $u$ without specifying $\lambda, \lambda^{\prime \prime}$. In particular, this element satisfies the requirements of (1), proving existence and uniqueness.

This argument also proves (2), since in this case we may pick $\lambda^{\prime}$ so that $b \in \mathcal{B}\left(\lambda+\lambda^{\prime}\right)$ and $b \in \mathcal{B}\left(\lambda^{\prime}+\lambda^{\prime \prime}\right)$, and then $t\left(\left(b \diamond b^{\prime \prime}\right)_{\lambda+\lambda^{\prime}, \lambda^{\prime}+\lambda^{\prime \prime}}\right)=0$.

The bar-invariance of the canonical basis of $N\left(\lambda, \lambda^{\prime \prime}\right)$ and the uniqueness of the element $u$ shows that $\bar{u}=u$, and hence (3) holds.

Finally, the uniqueness of $u$ forces $b \mho_{\zeta} b^{\prime \prime}=b^{-} b^{\prime \prime+} 1_{\zeta}$ modulo $P\left(\right.$ ht $|b|-1$, ht $\left.\left|b^{\prime \prime}\right|-1\right)$ Since $b^{-} b^{\prime \prime+} 1_{\zeta}$ forms a basis of $\dot{\mathbf{U}}$, the transition matrix from $b^{-} b^{\prime \prime+} 1_{\zeta}$ to $b \diamond_{\zeta} b^{\prime \prime}$ may be made upper unitriangular (by a suitable ordering), hence $b \diamond_{\zeta} b^{\prime \prime}$ forms a basis.

We let $\dot{\mathcal{B}}=\left\{\left(b \diamond_{\zeta} b^{\prime}:\left(b, b^{\prime}\right) \in \mathcal{B} \times_{\pi} \mathcal{B}, \zeta \in X\right\}\right.$ and call this the canonical basis of $\dot{\mathbf{U}}$.

Corollary 4.15. Let $\zeta \in X$ and $\left(b, b^{\prime}\right) \in \mathcal{B} \times_{\pi} \mathcal{B}$. For any $\lambda, \lambda^{\prime} \in X^{+}$such that $b \in B(\lambda)$, $b^{\prime} \in B\left(\lambda^{\prime}\right)_{\nu}$, and $\zeta=\lambda-\lambda^{\prime}$,

$$
\Delta_{4}\left(b \diamond_{\zeta} b^{\prime}\right)\left(\eta_{\lambda} \otimes \xi_{-\lambda^{\prime}}\right)=\pi^{\langle\tilde{\nu}, \lambda\rangle}\left(b \diamond_{4} b^{\prime}\right)_{\lambda, \lambda^{\prime}} .
$$

Proof. Let $u=b \diamond_{\zeta} b^{\prime}$. Then by definition, $\Delta(u) \eta_{\lambda} \otimes \xi_{-\lambda^{\prime \prime}}=\left(b \diamond b^{++}\right)_{\lambda, \lambda^{\prime}}$, and $\left(b \diamond b^{\prime}\right)_{\lambda, \lambda^{\prime}}-$ $b^{+} \eta_{\lambda} \otimes b^{-} \xi_{-\lambda^{\prime}} \in v \mathcal{L}$. Therefore, applying the isomorphism in Lemma 3.8 (which obviously preserves $\mathcal{L}$ ),

$$
\Delta_{4}(u) \eta_{\lambda} \otimes \xi_{-\lambda^{\prime \prime}}-\pi^{\langle\tilde{\nu}, \lambda\rangle} b^{+} \eta_{\lambda} \otimes b^{-} \xi_{-\lambda^{\prime}} \in v \mathcal{L} .
$$

On the other hand, $\Psi_{4}\left(\Delta_{4}(u) \eta_{\lambda} \otimes \xi_{-\lambda^{\prime \prime}}\right)=\Delta_{4}(u) \eta_{\lambda} \otimes \xi_{-\lambda^{\prime \prime}}$ by the bar-invariance of $u$. Then by uniqueness, we have $\Delta_{4}(u)=\pi^{\langle\tilde{\nu}, \lambda\rangle}\left(b \diamond_{4} b^{\prime}\right)_{\lambda, \lambda^{\prime}}$.

Example 4.16. Suppose that $I=I_{\overline{1}}=\{i\}$. Then $\mathcal{B}=\left\{\pi^{\epsilon} \theta_{i}^{(a)}: a \in \mathbb{N}, \epsilon \in\{0,1\}\right\}$. Let $a, b \in \mathbb{N}$ and suppose that $n \geq a+b$. Then using similar computations as in the proof of 
CW, Theorem 6.2],

$$
\begin{aligned}
\left(\theta_{i}^{(a)} \diamond_{n-2 a} \theta_{i}^{(b)}\right) & =F_{i}^{(a)} E_{i}^{(b)} 1_{n} \\
\left(\theta_{i}^{(a)} \triangleright_{2 b-n} \theta_{i}^{(b)}\right) & =\pi^{a b} E_{i}^{(b)} F_{i}^{(a)} 1_{-n} .
\end{aligned}
$$

We note that the though this $\pi$-basis matches the one in loc. cit., the $\left(\mathcal{B} \times_{\pi} \mathcal{B}\right) \times X$ labeling differs because several conventions differ. In particular, this canonical basis agrees with the categorical canonical basis produced in $[\mathrm{EL}$.

\section{INNER PRODUCT ON U்}

In this section, we will construct a bilinear form on $\dot{\mathbf{U}}$. This bilinear form is $\omega$-invariant and $\rho$-invariant, and can be viewed as a limit of bilinear forms on the vector spaces $N(\lambda, \lambda)^{\prime}$. In particular, the canonical basis is shown to be $\pi$-almost-orthonormal with respect to this form.

5.1. Review of automorphisms. Let us recall some additional automorphisms of $\mathbf{U}$ (and $\dot{\mathbf{U}}$ ) which will play a role in the following computations. We already defined $\omega$ and $\rho$ in 2.3 .

Let $\tau_{1}: \mathbf{U} \rightarrow \mathbf{U}$ be the anti-involution defined by

$$
\tau_{1}\left(E_{i}\right)=v_{i}^{-1} \tilde{K}_{-i} F_{i}, \quad \tau_{1}\left(F_{i}\right)=v_{i}^{-1} \tilde{K}_{i} E_{i}, \quad \tau_{1}\left(K_{\mu}\right)=K_{\mu}, \quad \tau_{1}\left(J_{\mu}\right)=J_{\mu} .
$$

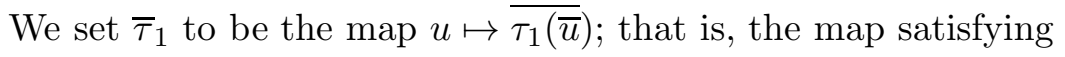

$$
\bar{\tau}_{1}\left(E_{i}\right)=\pi_{i} v_{i}^{-1} F_{i} \tilde{J}_{i} \tilde{K}_{i}, \quad \tau_{1}\left(F_{i}\right)=\pi_{i} v_{i}^{-1} E_{i} \tilde{J}_{i} \tilde{K}_{-i}, \quad \tau_{1}\left(K_{\mu}\right)=K_{\mu}, \quad \tau_{1}\left(J_{\mu}\right)=J_{\mu} .
$$

Lemma 5.1. We have the identities

$$
\rho \tau_{1}=\bar{\tau}_{1} \rho, \quad \omega^{-1} \tau_{1}=\tau_{1} \omega .
$$

Proof. It suffices to check these identities on the generators, and therefore, since all compositions considered fix $J_{\mu}$ and $K_{\mu}$, we only need to check on $E_{i}$ and $F_{i}$ for $i \in I$.

Well,for $\rho \tau_{1}=\bar{\tau}_{1} \rho$ :

$$
\begin{gathered}
\rho\left(\tau_{1}\left(E_{i}\right)\right)=\rho\left(v_{i}^{-1} \tilde{K}_{-i} F_{i}\right)=v_{i}^{-1} F_{i} \tilde{K}_{i}, \quad \bar{\tau}_{1}\left(\rho\left(E_{i}\right)=\bar{\tau}_{1}\left(\pi_{i} \tilde{J}_{i} E_{i}\right)=v_{i}^{-1} F_{i} \tilde{K}_{i},\right. \\
\rho\left(\tau_{1}\left(F_{i}\right)\right)=\rho\left(v_{i}^{-1} \tilde{K}_{i} E_{i}\right)=\pi_{i} v_{i}^{-1} E_{i} \tilde{J}_{i} \tilde{K}_{-i}, \quad \bar{\tau}_{1}\left(\rho\left(F_{i}\right)=\bar{\tau}_{1}\left(E_{i}\right)=v_{i}^{-1} E_{i} \tilde{J}_{i} \tilde{K}_{-i} .\right.
\end{gathered}
$$

Checking the left-hand equations to the right-hand equations verifies the identity.

Similarly, for $\omega^{-1} \tau_{1}=\tau_{1} \omega$ :

$$
\begin{gathered}
\omega^{-1}\left(\tau_{1}\left(E_{i}\right)\right)=\omega^{-1}\left(v_{i}^{-1} \tilde{K}_{-i} F_{i}\right)=v_{i}^{-1} \tilde{K}_{i} E_{i}, \quad \tau_{1}\left(\omega\left(E_{i}\right)\right)=\tau_{1}\left(F_{i}\right)=v_{i}^{-1} \tilde{K}_{i} E_{i}, \\
\omega^{-1}\left(\tau_{1}\left(F_{i}\right)\right)=\omega\left(v_{i}^{-1} \tilde{K}_{i} E_{i}\right)=\pi_{i} v_{i}^{-1} \tilde{J}_{i} \tilde{K}_{-i} F_{i}, \quad \tau_{1}\left(\omega\left(F_{i}\right)=\tau_{1}\left(\pi_{i} \tilde{J}_{i} E_{i}\right)=\pi_{i} v_{i}^{-1} \tilde{J}_{i} \tilde{K}_{-i} F_{i} .\right.
\end{gathered}
$$

Comparing the left-hand equations to the right-hand equations verifies the identity.

5.2. The bilinear form. Let $(\cdot, \cdot): \mathbf{f} \times \mathbf{f} \rightarrow \mathbb{Q}(v)^{\pi}$ denote the bilinear form $\{\cdot, \cdot\}$ in CHW1. Let $\tau_{1}: \mathbf{U} \rightarrow \mathbf{U}$ be as defined in CHW2, and note it induces a homomorphism $\tau_{1}: \dot{\mathbf{U}} \rightarrow \dot{\mathbf{U}}$. Let $\bar{\tau}_{1}$ be the bar-conjugate homomorphism.

Theorem 5.2. There exists a unique $\mathbb{Q}(v)^{\pi}$-bilinear pairing $(\cdot, \cdot): \dot{\mathbf{U}} \times \dot{\mathbf{U}} \rightarrow \mathbb{Q}(v)^{\pi}$ such that the following hold.

(1) $\left(1_{\lambda_{1}} x 1_{\lambda_{2}}, 1_{\lambda_{1}^{\prime}} x^{\prime} 1_{\lambda_{2}^{\prime}}\right)$ is zero unless $\lambda_{1}=\lambda_{1}^{\prime}, \lambda_{2}=\lambda_{2}^{\prime}$;

(2) $(u x, y)=\left(x, \tau_{1}(u) y\right)$ for all $x, y \in \dot{\mathbf{U}}$ and $u \in \mathbf{U}$;

(3) $\left(x^{-} 1_{\lambda}, x^{\prime-} 1_{\lambda}\right)=\left(x, x^{\prime}\right)$ for all $x, x^{\prime} \in \mathbf{f}$ and all $\lambda$;

(4) We have $(x, y)=(y, x)$.

Proof. The proof is essentially the same as in [L93, Theorem 26.1.2]. 
Proposition 5.3. We have $(x u, y)=\left(x, y \bar{\tau}_{1}(u)\right)$ for all $x, y \in \dot{\mathbf{U}}, u \in \mathbf{U}$.

Proof. It suffices to prove this for the generators; it is clear for $K_{\mu}, J_{\mu}$. It remains to verify that

$$
\left(x E_{i}, y\right)=\left(x, \pi_{i} v_{i}^{-1} y F_{i} \tilde{J}_{i} \tilde{K}_{i}\right), \quad\left(x F_{i}, y\right)=\left(x, \pi_{i} v_{i}^{-1} y E_{i} \tilde{J}_{i} \tilde{K}_{-i}\right) .
$$

We may further assume that $x=u^{\prime} 1_{\zeta}$ where $u^{\prime} \in U$ and $\zeta \in X$. Then setting $y^{\prime}=\tau_{1}\left(u^{\prime}\right) y$, we see that the previous equalities follow from

$$
\left(1_{\zeta} E_{i}, y^{\prime}\right)=\left(1_{\zeta}, \pi_{i} v_{i}^{-1} y^{\prime} F_{i} \tilde{J}_{i} \tilde{K}_{i}\right), \quad\left(x F_{i}, y\right)=\left(x, \pi_{i} v_{i}^{-1} y E_{i} \tilde{J}_{i} \tilde{K}_{-i}\right) .
$$

Once more, we can assume that $y^{\prime}=\tau_{1}\left(y_{1}^{-}\right) y_{2}^{-} 1_{\zeta}^{\prime}$ for homogeneous $y_{1}, y_{2} \in \mathbf{f}$, so it suffices to show that

$$
\begin{aligned}
& \left(y_{1}^{-} E_{i} 1_{\zeta-i^{\prime}}, y_{2}^{-} 1_{\zeta}^{\prime}\right)=\pi_{i}^{1+\left\langle i, \zeta^{\prime}+i^{\prime}\right\rangle} v_{i}^{-1+\left\langle i, \zeta^{\prime}+i^{\prime}\right\rangle}\left(y_{1}^{-} 1_{\zeta}, y_{2}^{-} F_{i} 1_{\zeta^{\prime}+i^{\prime}}\right) \\
& \left(y_{1}^{-} F_{i} 1_{\zeta+i^{\prime}}, y_{2}^{-} 1_{\zeta}^{\prime}\right)=\pi_{i}^{1+\left\langle i, \zeta^{\prime}-i^{\prime}\right\rangle} v_{i}^{-1-\left\langle i, \zeta^{\prime}-i^{\prime}\right\rangle}\left(y_{1}^{-} 1_{\zeta}, y_{2}^{-} E_{i} 1_{\zeta^{\prime}-i^{\prime}}\right)
\end{aligned}
$$

By symmetry, (a) and (b) are equivalent, so we shall prove (a). Then we may assume $\zeta^{\prime}=\zeta-i^{\prime}$ and $\left|y_{1}\right|=\left|y_{2}\right|+i$.

Recall the $\mathbb{Q}(v)^{\pi}$-linear differentials ${ }_{i} r, r_{i}: \mathbf{f} \rightarrow \mathbf{f}$ defined by ${ }_{i} r\left(\theta_{j}\right)=r_{i}\left(\theta_{j}\right)=\delta_{i j}$,

$$
{ }_{i} r(x y)={ }_{i} r(x) y+\pi^{p(x) p(i)} v^{-i \cdot|x|} x_{i} r(y), \quad r_{i}(x y)=\pi^{p(x) p(i)} v^{-i \cdot|x|} r_{i}(x) y+x r_{i}(y) .
$$

Using a variant on [CHW1, Proposition 2.2.2] (essentially with $q$ replaced by $v^{-1}$ ) we have

$$
E_{i} y_{1}^{-} 1_{\zeta^{\prime}}-\pi_{i}^{p\left(y_{1}\right)} y_{1}^{-} E_{i} 1_{\zeta^{\prime}}=\frac{\pi_{i}^{p\left(y_{1}\right)-p(i)} r_{i}\left(y_{1}\right)^{-} \tilde{J}_{i} \tilde{K}_{i}-\tilde{K}_{-i}{ }_{i} r\left(y_{1}\right)^{-}}{\pi_{i} v_{i}-v_{i}^{-1}} 1_{\zeta},
$$

from whence we see that

$$
y_{1}^{-} E_{i} 1_{\zeta^{\prime}}=\pi_{i}^{p\left(y_{1}\right)} E_{i} y_{1}^{-} 1_{\zeta^{\prime}}+\frac{\pi_{i} r_{i}\left(y_{1}\right)^{-} \tilde{J}_{i} \tilde{K}_{i}-\pi_{i}^{p\left(y_{1}\right)} \tilde{K}_{-i}{ }_{i} r\left(y_{1}\right)^{-}}{v_{i}^{-1}-\pi_{i} v_{i}} 1_{\zeta} .
$$

Now note that

$$
\left(\pi_{i}^{p\left(y_{1}\right)} E_{i} y^{-} 1_{\zeta^{\prime}}, y_{2}^{-} 1_{\zeta^{\prime}}\right)=\pi_{i}^{p\left(y_{1}\right)} v_{i}^{1+\left\langle i,\left|y_{2}\right|-\zeta^{\prime}\right\rangle}\left(y_{1}^{-} 1_{\zeta^{\prime}}, F_{i} y_{2}^{-} 1_{\zeta^{\prime}}\right)=\pi_{i}^{p\left(y_{1}\right)} v_{i}^{1+\left\langle i,\left|y_{2}\right|-\zeta^{\prime}\right\rangle}\left(y_{1}, \theta_{i} y_{2}\right)
$$

and that

$$
\begin{aligned}
& \left(\frac{\pi_{i} r_{i}\left(y_{1}\right)^{-} \tilde{J}_{i} \tilde{K}_{i}-\pi_{i}^{p\left(y_{1}\right)} \tilde{K}_{-i}{ }_{i} r\left(y_{1}\right)^{-}}{v_{i}^{-1}-\pi_{i} v_{i}} 1_{\zeta}, y_{2}^{-} 1_{\zeta^{\prime}}\right) \\
& =\left(\theta_{i}, \theta_{i}\right)\left(\pi_{i}^{1+\left\langle i, \zeta^{\prime}\right\rangle} v_{i}^{1+\left\langle i, \zeta^{\prime}\right\rangle} r_{i}\left(y_{1}\right)^{-} 1_{\zeta^{\prime}}-\pi_{i}^{p\left(y_{1}\right)} v_{i}^{1+\left\langle i,\left|y_{1}\right|-i-\zeta^{\prime}\right\rangle}{ }_{i} r\left(y_{1}\right)^{-} 1_{\zeta^{\prime}}, y_{2}^{-} 1_{\zeta^{\prime}}\right) \\
& =\pi_{i}^{1+\left\langle i, \zeta^{\prime}\right\rangle} v_{i}^{1+\left\langle i, \zeta^{\prime}\right\rangle}\left(\theta_{i}, \theta_{i}\right)\left(r_{i}\left(y_{1}\right), y_{2}\right)-\pi_{i}^{p\left(y_{1}\right)} v_{i}^{1+\left\langle i,\left|y_{1}\right|-i-\zeta^{\prime}\right\rangle}\left(\theta_{i}, \theta_{i}\right)\left({ }_{i} r\left(y_{1}\right), y_{2}\right) \\
& =\pi_{i}^{1+\left\langle i, \zeta^{\prime}\right\rangle} v_{i}^{1+\left\langle i, \zeta^{\prime}\right\rangle}\left(y_{1}, y_{2} \theta_{i}\right)-\pi_{i}^{p\left(y_{1}\right)} v_{i}^{1+\left\langle i,\left|y_{1}\right|-i-\zeta^{\prime}\right\rangle}\left(y_{1}, \theta_{i} y_{2}\right) .
\end{aligned}
$$

Then we see that

$\left(y_{1}^{-} E_{i} 1_{\zeta^{\prime}}, y_{2}^{-} 1_{\zeta}\right)=\pi_{i}^{1+\left\langle i, \zeta^{\prime}\right\rangle} v_{i}^{1+\left\langle i, \zeta^{\prime}\right\rangle}\left(y_{1}, y_{2} \theta_{i}\right)=\pi^{1+\left\langle i, \zeta^{\prime}+i^{\prime}\right\rangle} v_{i}^{-1+\left\langle i, \zeta^{\prime}+i^{\prime}\right\rangle}\left(y_{1}^{-} 1_{\zeta^{\prime}+i}, y_{2}^{-} F_{i} 1_{\zeta^{\prime}+i^{\prime}}\right)$, which proves (a).

Proposition 5.4. We have $(\rho(x), \rho(y))=(x, y)$ for all $x, y \in \dot{\mathbf{U}}$.

Proof. It suffices to show that $x, y \mapsto\left(\rho(x), \rho^{\prime}(y)\right)$ satisfies the defining properties of $(\cdot, \cdot)$. All of these are obvious except Theorem $5.2(2)$. However, this follows from the previous proposition and the fact that $\rho \tau_{1}=\bar{\tau}_{1} \rho$.

Lemma 5.5. For $x, x^{\prime} \in \mathbf{f}_{\nu}, \nu \in N[I]$ and $\lambda \in X,\left(x^{+} 1_{\lambda}, x^{\prime+} 1_{\lambda}\right)=\pi_{\nu} \pi^{\langle\tilde{\nu}, \lambda\rangle}\left(x, x^{\prime}\right)$. 
Proof. We have

$\left(x^{+} 1_{\lambda}, x^{\prime+} 1_{\lambda}\right)=\left(1_{\lambda}, \tau_{1}\left(x^{+}\right) x^{\prime+} 1_{\lambda}\right)=\left(1_{-\lambda}, 1_{-\lambda} \rho\left(x^{\prime+}\right) \rho \tau_{1}\left(x^{+}\right)\right)=\left(1_{-\lambda}, \rho\left(x^{\prime+}\right) \bar{\tau}_{1} \rho\left(x^{+}\right) 1_{-\lambda}\right)$.

Then we may rearrange to obtain

$$
\left(x^{+} 1_{\lambda}, x^{\prime+} 1_{\lambda}\right)=\left(\tau_{1} \rho\left(x^{\prime+}\right) 1_{-\lambda}, \bar{\tau}_{1} \rho\left(x^{+}\right) 1_{-\lambda}\right) .
$$

Now note that for any $x \in \mathbf{f}_{\nu}, \tau_{1} \rho\left(x^{+}\right)=\pi_{\nu} v_{\nu} v^{\bullet(\nu)} \rho(x)^{-} \tilde{J}_{\nu} \tilde{K}_{-\nu}$ where $\bullet\left(i_{1}+\ldots+i_{n}\right)=$ $\sum_{s<t} i_{s} \cdot i_{t}$. Likewise, noting that $\bullet(\nu) \in 2 \mathbb{Z}$, we obtain $\bar{\tau}_{1} \rho\left(x^{+}\right)=v_{-\nu} v^{-\bullet(\nu)} \rho(x)^{-} \tilde{K}_{\nu}$. Then

$$
\left(\tau_{1} \rho\left(x^{\prime+}\right) 1_{-\lambda}, \bar{\tau}_{1} \rho\left(x^{+}\right) 1_{-\lambda}\right)=\pi_{\nu} \pi^{\langle\tilde{\nu}, \lambda\rangle}\left(x, x^{\prime}\right)
$$

Proposition 5.6. We have $\left(\omega(x), \omega^{-1}(y)\right)=(x, y)$ for all $x, y \in \dot{\mathbf{U}}$.

Proof. It suffices to show that $x, y \mapsto\left(\omega(x), \omega^{-1}(y)\right)$ satisfies the defining properties of $(\cdot, \cdot)$. It is clear that (1) holds. Since $\omega \tau_{1}=\tau_{1} \omega$, it is clear that (2) is satisfied. Note that $\omega\left(x^{-} 1_{\lambda}\right)=\pi_{\nu} \pi^{\langle\tilde{\nu}, \lambda\rangle} x^{+} 1_{\lambda}$, while $\omega^{-1}\left(x^{\prime-} 1_{\lambda}\right)=x^{\prime+} 1_{\lambda}$ for each $x, x^{\prime} \in \mathbf{f}_{\nu}$, whence $(3)$ holds by the previous lemma. Finally, we note that $\omega^{2}(y)=\pi_{\nu} \pi^{\langle\tilde{\nu}, \lambda\rangle} x$ for any $x \in \dot{\mathbf{U}} 1_{\lambda}$, and hence $\left(\omega^{2}(x), \omega^{2}(y)\right)=(x, y)$ for any $x, y \in \dot{\mathbf{U}}$, proving (4).

Example 5.7. We compute the following inner products. Let $\lambda_{i}=\langle i, \lambda\rangle$.

$$
\begin{aligned}
& \left(F_{i}^{(k)} 1_{\lambda}, F_{i}^{(k)} 1_{\lambda}\right)=\pi^{\left(\begin{array}{c}
k \\
2
\end{array}\right)} \prod_{s=1}^{k} \frac{1}{1-\left(\pi_{i} v_{i}^{2}\right)^{s}} \\
& \left(E_{i}^{(k)} 1_{\lambda}, E_{i}^{(k)} 1_{\lambda}\right)=\pi_{i}^{\left(\begin{array}{c}
k+1 \\
2
\end{array}\right)+k \lambda_{i}} \prod_{s=1}^{k} \frac{1}{1-\left(\pi_{i} v_{i}^{2}\right)^{s}} \\
& \left(E_{i} F_{i} 1_{\lambda}, 1_{\lambda}\right)=\frac{v_{i}^{1-\lambda_{i}}}{1-\pi_{i} v_{i}^{2}} \\
& \left(E_{i} F_{i} 1_{\lambda}, E_{i} F_{i} 1_{\lambda}\right)=\pi^{\lambda_{i}-1} \frac{1+\left(\pi_{i} v_{i}^{2}\right)^{1-\lambda_{i}}}{\left(1-\pi_{i} v_{i}^{2}\right)^{2}} \\
& \left(E_{i} F_{i} 1_{\lambda}, F_{i} E_{i} 1_{\lambda}\right)=\pi^{\lambda_{i}} \frac{1+\pi_{i} v_{i}^{2}}{\left(1-\pi_{i} v_{i}^{2}\right)^{2}}
\end{aligned}
$$

We note that under the identification $q^{2}=\pi v^{2}$, these values are formally similar to the values of the analogous bilinear form on $\left.\dot{\mathbf{U}}\right|_{\pi=1}$ over $\mathbb{Q}(q)$ (but with an additional factor of some power of $\pi$ ).

Proposition 5.8. Let $\tau_{1}^{\prime}$ be the $\mathbb{Q}(v)^{\pi}$-linear anti-automorphism of $\mathbf{U}$ defined by

$$
\tau_{1}^{\prime}\left(E_{i}\right)=v_{i} \tilde{K}_{i} F_{i}, \quad \tau_{1}^{\prime}\left(F_{i}\right)=v_{i} \tilde{K}_{i}^{-1} E_{i}, \quad \tau_{1}^{\prime}\left(K_{\nu}\right)=K_{\nu}, \quad \tau_{1}^{\prime}\left(J_{\nu}\right)=J_{\nu} .
$$

Then there is a $\mathbb{Q}(v)^{\pi}$-bilinear pairing $(\cdot, \cdot)^{\prime}: \dot{\mathbf{U}} \times \dot{\mathbf{U}} \rightarrow \mathbb{Q}(v)^{\pi}$ such that the following hold.

(1) $\left(1_{\lambda_{1}} x 1_{\lambda_{2}}, 1_{\lambda_{1}^{\prime}} x^{\prime} 1_{\lambda_{2}^{\prime}}\right)^{\prime}$ is zero unless $\lambda_{1}=\lambda_{1}^{\prime}, \lambda_{2}=\lambda_{2}^{\prime}$;

(2) $(u x, y)^{\prime}=\left(x, \tau_{1}^{\prime}(u) y\right)^{\prime}$ for all $x, y \in \dot{\mathbf{U}}$ and $u \in \mathbf{U}$;

(3) $\left(x^{-} 1_{\lambda}, x^{-} 1_{\lambda}\right)^{\prime}=\overline{\left(\bar{x}, \overline{x^{\prime}}\right)}$ for all $x, x^{\prime} \in \mathbf{f}$ and all $\lambda$;

(4) We have $(x, y)^{\prime}=(y, x)^{\prime}$.

Moreover, we have $\left(\omega(x), \omega^{-1}(y)\right)^{\prime}=(\rho(x), \rho(y))^{\prime}=(x, y)^{\prime}$. 


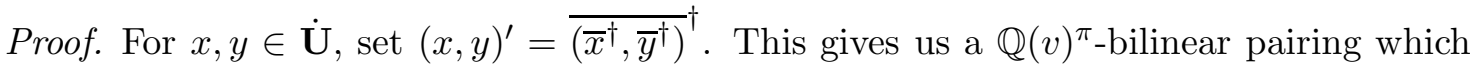
clearly satisfies (1) and (4). (2) follows from the observation that ${\overline{\tau_{1}}}^{\prime}\left(\bar{u}^{\dagger}\right)^{\dagger}=\tau_{1}(u)$.

For (3), by Theorem $5.2(3)$ we have $\left(x^{-} 1_{\lambda}, x^{\prime-} 1_{\lambda}\right)^{\prime}=\left(x^{\dagger}, x^{\prime \dagger}\right)^{\dagger}$. However, it is easy to check that the bilinear form $(\cdot, \cdot)^{\prime}: \mathbf{f} \times \mathbf{f} \rightarrow \mathbb{Q}(v)^{\pi}$ defined by $(x, y)^{\prime}=\left(x^{\dagger}, y^{\dagger}\right)^{\dagger}$ for $x, y \in \mathbf{f}$ satisfies the defining properties of $(\cdot, \cdot)$, hence $(x, y)^{\prime}=(x, y)$.

Lastly, we note that $\omega\left(\bar{u}^{\dagger}\right)={\overline{\omega^{-1}(u)}}^{\dagger}$ and $\rho\left(\bar{u}^{\dagger}\right)={\overline{\left(\omega \rho \omega^{-1}\right)(u)}}^{\dagger}$, so $\rho$ - and $\omega$-invariance follows from the properties of $(\cdot, \cdot)$.

Remark 5.9. Suppose $I=I_{\overline{1}}=\{i\}$. In [CW], the bilinear form $(\cdot, \cdot)^{\prime}$ is defined on $\dot{\mathbf{U}}$. With respect to this form, $\left(E^{(a)} 1_{n}, E^{(a)} 1_{n}\right)^{\prime}$ is not $\pi$-almost-orthonormal (with respect to $v^{-1}$ ) in general, a fact which is not desirable from a categorification standpoint. (However, in light of Remark 2.8. Proposition 5.8 demonstrates that $(\cdot, \cdot)^{\prime}$ is well suited to the $\dagger$-twisted U.) In this regard, the bilinear form defined in Theorem 5.2 is a better choice.

Alternatively, Ellis and Lauda [EL] have used a dagger-sesquilinear variant of $(\cdot, \cdot)^{\prime}$ in their categorification. Specifically, the Ellis-Lauda form $(\cdot, \cdot)_{\mathrm{EL}}$ seems to relate to ours via

$$
(x, y)_{\mathrm{EL}}=\left(\omega(x)^{\dagger}, \omega^{-1}(y)\right)^{\prime} .
$$

5.3. The bilinear form as a limit. Let $\zeta \in X$ and $\lambda, \lambda^{\prime} \in X^{+}$such that $\lambda-\lambda^{\prime}=\zeta$. From [CHW2], the module $V(\lambda)$ is equipped with a bilinear form $(\cdot, \cdot)_{\lambda}$ such that

$$
(u x, y)_{\lambda}=\left(x, \tau_{1}(u) y\right)_{\lambda} \text { for all } x, y \in V(\lambda), u \in \mathbf{U} .
$$

Such a bilinear form is called a polarization. It is also shown in loc. cit. that while there is no natural polarization on $V(\lambda) \otimes V\left(\lambda^{\prime}\right)$, there is an induced bilinear pairing between $V(\lambda) \otimes V\left(\lambda^{\prime}\right)$ and $V(\lambda) \otimes_{4} V\left(\lambda^{\prime}\right)$, called a $J$-polarization; that is, a bilinear form $(\cdot, \cdot):\left(V(\lambda) \otimes V\left(\lambda^{\prime}\right)\right) \times\left(V(\lambda) \otimes V\left(\lambda^{\prime}\right)\right) \rightarrow \mathbb{Q}(v)^{\pi}$ satisfying

$$
\left(\Delta_{3}(u) x, y\right)=\left(x, \Delta_{4}\left(\tau_{1}(u)\right) y\right) \text { for all } x, y \in V(\lambda), u \in \mathbf{U} .
$$

We will use the following variant of this construction.

Proposition 5.10. Consider the bilinear pairing $(\cdot, \cdot)_{\lambda, \lambda^{\prime}}: N\left(\lambda, \lambda^{\prime}\right) \times N\left(\lambda, \lambda^{\prime}\right) \rightarrow \mathbb{Q}(v)^{\pi}$ defined by setting

$$
\left(x \otimes x^{\prime}, y \otimes y^{\prime}\right)_{\lambda, \lambda^{\prime}}=\pi_{\nu} \pi^{\left\langle\tilde{\nu}, \lambda^{\prime}\right\rangle}(x, y)_{\lambda}\left(x^{\prime}, y^{\prime}\right)_{\lambda^{\prime}}
$$

for homogeneous $x, x^{\prime} \in V(\lambda)$ and $y, y^{\prime} \in V\left(\lambda^{\prime}\right)$ and $y \in V\left(\lambda^{\prime}\right)_{\lambda-\nu^{\prime}}$ for some $\nu \in \mathbb{N}[I]$. Then $(\cdot, \cdot)_{\lambda, \lambda^{\prime}}$ is a J-polarization.

Proof. This is an elementary verification akin to [CHW2, Proof of Lemma 4.9]; we note that the $\pi_{\nu} \pi^{\left\langle\tilde{\nu}, \lambda^{\prime}\right\rangle}$ factor is related to Lemma 5.5.

Proposition 5.11. Let $x, y \in \dot{\mathbf{U}} 1_{\zeta}$. When the pair $\lambda, \lambda^{\prime}$ tends to $\infty$ (in the sense that $\langle i, \lambda\rangle$ tends to $\infty$ for all $i)$, the inner product $\left(x\left(\eta_{\lambda} \otimes \zeta_{-\lambda^{\prime}}\right), y\left(\eta_{\lambda} \otimes \zeta_{-\lambda^{\prime}}\right)\right)_{\lambda, \lambda^{\prime}}$ converges in $\mathbb{Q}^{\pi}((v))$ to $(x, y)$.

Proof. Assume first that $x=x_{1}^{-} 1_{\zeta}$ and $y=y_{1}^{-} 1_{\zeta}$ for $x_{1}, y_{1} \in \mathbf{f}$ Then

$$
\begin{aligned}
& x\left(\eta_{\lambda} \otimes \zeta_{-\lambda^{\prime}}\right)=x_{1}^{-} \eta_{\lambda} \otimes \zeta_{-\lambda^{\prime}}, \\
& y\left(\eta_{\lambda} \otimes \zeta_{-\lambda^{\prime}}\right)=y_{1}^{-} \eta_{\lambda} \otimes \zeta_{-\lambda^{\prime}} .
\end{aligned}
$$

Therefore $\left(x\left(\eta_{\lambda} \otimes \zeta_{-\lambda^{\prime}}\right), y\left(\eta_{\lambda} \otimes \zeta_{-\lambda^{\prime}}\right)\right)_{\lambda, \lambda^{\prime}}=\left(x \eta_{\lambda}, y \eta_{\lambda}\right)_{\lambda}$, and the right-hand side converges to $(x, y)=\left(x^{-} 1_{\zeta}, y^{-} 1_{\zeta}\right)$ by [CHW2, Proposition 6.10].

Now assume that $x=1_{\zeta}$ and $y$ is arbitrary. Then we may assume that $y=\tau_{1}\left(x_{1}^{-}\right) y_{1}^{-} 1_{\zeta}$ for some $x_{1}, x_{2} \in \mathbf{f}$. But then by the polarization property,

$$
\left(1_{\zeta}\left(\eta_{\lambda} \otimes \zeta_{-\lambda^{\prime}}\right), y\left(\eta_{\lambda} \otimes \zeta_{-\lambda^{\prime}}\right)\right)_{\lambda, \lambda^{\prime}}=\left(x_{1}^{-}\left(\eta_{\lambda} \otimes \zeta_{-\lambda^{\prime}}\right), y_{1}^{-}\left(\eta_{\lambda} \otimes \zeta_{-\lambda^{\prime}}\right)\right) .
$$


Using the previous case, this converges to

$$
\left(x_{1}^{-} 1_{\zeta}, y_{1}^{-} 1_{\zeta}\right)=\left(1_{\zeta}, \tau_{1}\left(x_{1}^{-}\right) y_{1}^{-} 1_{\zeta}\right)=(x, y)
$$

Finally, let us assume $x$ and $y$ are both arbitrary. We may assume that $x=u 1_{\zeta}$ for some $u \in \mathbf{U}$. Then

$\left(x\left(\eta_{\lambda} \otimes \zeta_{-\lambda^{\prime}}\right), y\left(\eta_{\lambda} \otimes \zeta_{-\lambda^{\prime}}\right)\right)_{\lambda, \lambda^{\prime}}=\left(u\left(\eta_{\lambda} \otimes \zeta_{-\lambda^{\prime}}\right), y\left(\eta_{\lambda} \otimes \zeta_{-\lambda^{\prime}}\right)\right)_{\lambda, \lambda^{\prime}}=\left(1_{\zeta}\left(\eta_{\lambda} \otimes \zeta_{-\lambda^{\prime}}\right), \tau_{1}(u) y\left(\eta_{\lambda} \otimes \zeta_{-\lambda^{\prime}}\right)\right)$.

Again, by the previous case, this converges to $\left(1_{\zeta}, \tau_{1}(u) y\right)=(x, y)$.

Given $\mathbb{Q}(v)^{\pi}$-modules $M, M^{\prime}$ and a pairing $(\cdot, \cdot): M \times M^{\prime} \rightarrow \mathbb{Q}(v)^{\pi}$, we say a $\pi$-basis $B$ of $M$ is $\pi$-almost-orthonormal to a $\pi$-basis $B^{\prime}$ of $M^{\prime}$ if they satisfy the following conditions:

$$
\begin{gathered}
\left(b, b^{\prime}\right) \in \mathbb{Z}^{\pi}[[v]] \cap \mathbb{Q}(v)^{\pi} ; \\
\left(b, b^{\prime}\right) \in v \mathbb{Z}^{\pi}[[v]] \text { if } b \neq b^{\prime} \text { and } b \neq \pi b^{\prime} ; \\
(b, b) \in \pi^{\epsilon}+v \mathbb{Z}^{\pi}[[v]] \text { for some } \epsilon \in\{0,1\} .
\end{gathered}
$$

In the case $M=M^{\prime}$, we will just say $\pi$-almost-orthonormal.

Theorem 5.12. The basis $\dot{\mathcal{B}}$ is $\pi$-almost-orthonormal.

Proof. It is trivial that $\left(b \diamond b^{\prime}, b_{1} \diamond_{\zeta^{\prime}} b_{1}^{\prime}\right)=0$ if $\zeta \neq \zeta^{\prime}$. In particular, we may assume the root datum is simply connected.

Recall by construction and by Corollary 4.15 that for $s=3,4$ and for all $\lambda, \lambda^{\prime} \in X^{+}$ such that if $\lambda-\lambda^{\prime}=\zeta$,

$$
\Delta_{s}\left(b \diamond_{\zeta} b^{\prime}\right) \eta_{\lambda} \otimes \xi_{-\lambda^{\prime}}=\pi^{\epsilon}\left(b \diamond_{s} b^{\prime}\right)_{\lambda, \lambda^{\prime}},
$$

where $\epsilon \in\{0,1\}$ (and by convention, we set $\left(b \diamond_{s} b^{\prime}\right)_{\lambda, \lambda^{\prime}}=0$ if $b \notin \mathcal{B}(\lambda)$ or $b^{\prime} \notin \mathcal{B}\left(\lambda^{\prime}\right)$ ).

By the previous proposition, it suffices to show that for all $\lambda, \lambda^{\prime} \in X^{+}$such that if $\lambda-\lambda^{\prime}=\zeta$ such that $b \in B(\lambda)$ and $b^{\prime} \in B\left(\lambda^{\prime}\right),\left(b \diamond b^{\prime}\right)_{\lambda, \lambda^{\prime}}$ is $\pi$-almost-orthonormal to $\left(b_{1} \diamond_{4} b_{1}^{\prime}\right)_{\lambda, \lambda^{\prime}}$ for all $b_{1}, b_{1}^{\prime}$. But this follows from the definition of the bilinear form and the almost- $\pi$-orthonomality of the canonical basis of $V(\lambda)$; cf. CHW2, Proposition 6.1].

Remark 5.13. It should be noted that, in contrast to the non-super case, a characterization of $\dot{\mathcal{B}}$ via $\pi$-almost-orthonormality is not possible, since in particular it is not possible for $\mathcal{B}$; cf. [CHW2, Example 6.3].

\section{TWISTORS}

In this section, we turn to some related constructions on $\dot{\mathbf{U}}$ which first appeared in CFLW]. Henceforth, we will assume that our datum is also $X$-regular (as defined in \$2.1). Let $\dot{\mathfrak{X}}$ be the twistor map on $\dot{\mathbf{U}}$ in [CFLW]. Then the complexification of $\left.\dot{\mathbf{U}}\right|_{\pi=-1}$ has a basis obtained by applying $\dot{\mathfrak{X}}$ to Lusztig's canonical basis of $\left.\dot{\mathbf{U}}\right|_{\pi=1}$. We shall show that the resulting basis equals the specialization of $\mathcal{B}$ up to a factor of an integral power of $\sqrt{-1}$. Along the way, we extend the notion of twistor maps to representations of $\mathbf{U}$.

6.1. The twistor maps. In this section, we recall (and restate) some results from CFLW]. Let $\mathbf{t}$ be a square root of -1 . For $n, m \in \mathbb{Z}$, we shall use the shorthand $n \equiv_{4} m$ if $n$ is congruent to $m$ modulo 4 . We will freely use the fact that if $n, m \in \mathbb{Z}$ such that $n \equiv{ }_{4} m$, then $\mathbf{t}^{n}=\mathbf{t}^{m}$.

Definition 6.1. An enhancer $\phi$ is an function $\phi: \mathbb{Z}[I] \times X \rightarrow \mathbb{Z}$ satisfying

(1) $\phi\left(\nu, \lambda+\mu^{\prime}\right) \equiv_{4} \phi\left(\nu, \mu^{\prime}\right)+\phi(\nu, \lambda)$ and $\phi(\nu+\mu, \lambda) \equiv_{4} \phi(\nu, \lambda)+\phi(\mu, \lambda)$ for $\nu, \mu \in \mathbb{Z}[I]$ and $\lambda \in X$.

(2) $\phi\left(i, j^{\prime}\right) \in 2 \mathbb{Z}$ for $i \neq j \in I$.

(3) $\phi\left(i, j^{\prime}\right)-\phi\left(j, i^{\prime}\right) \equiv_{4} i \cdot j+2 p(i) p(j)$ and $\phi\left(i, i^{\prime}\right) \equiv_{4} d_{i}$ for $i \neq j \in I$. 
We note that $\phi$ need not be $\mathbb{Z}$-linear in the second coordinate; specifically, it need not be the case that $\phi\left(\nu, \lambda+\lambda^{\prime}\right) \equiv_{4} \phi(\nu, \lambda)+\phi\left(\nu, \lambda^{\prime}\right)$ for $\lambda, \lambda^{\prime} \in X$.

Example 6.2. Let $I=I_{\overline{1}}=\{i\}$, so $X=\mathbb{Z}$ and $i^{\prime}=2 \in \mathbb{Z}$. Then an enhancer $\phi$ is determined entirely by choosing a value of $\phi(1,1)$; indeed, if $n, k \in \mathbb{Z}$ and $r \in\{0,1\}$ then

$$
\phi(n, 2 k+r) \equiv_{4} n \phi(1,2 k+r)=n \phi(1, r)+n k \phi(1,2),
$$

and we have $\phi(1,2) \equiv_{4} 1$ and $\phi(1,0) \equiv_{4} 0$ from the definition of an enhancer. In this case, there is no way for $\phi$ to be $\mathbb{Z}$-bilinear since $1 \equiv_{4} \phi(1,2) \not_{4} 2 \phi(1,1)$ for any choice of $\phi(1,1)$.

As shown in loc. cit., such an enhancer exists when the Cartan datum is $X$-regular. We shall henceforth assume an enhancer exists, and fix a choice $\phi$. We can extend scalars and enlarge the Cartan subalgebra of the quantum covering group as follows.

Definition 6.3. [CFLW, §4.4] The $\phi$-enhanced quantum covering group $\widehat{\mathbf{U}}$ associated to a super root datum $(Y, X, I, \cdot)$ and enhancer $\phi$ is the $\mathbb{Q}^{\pi}(\mathbf{t}, v)$-algebra with generators $E_{i}, F_{i}, K_{\mu}, J_{\mu}, T_{\mu}$, and $\Upsilon_{\mu}$, for $i \in I$ and $\mu \in Y$, subject to the relations (2.6)-(2.11), as well as the relations

$$
\begin{gathered}
T_{\mu} T_{\nu}=T_{\mu+\nu}, \quad \Upsilon_{\mu} \Upsilon_{\nu}=\Upsilon_{\mu+\nu}, \quad T_{0}=\Upsilon_{0}=T_{\nu}^{4}=\Upsilon_{\nu}^{4}=1, \quad T_{\mu} \Upsilon_{\nu}=\Upsilon_{\nu} T_{\mu}, \\
T_{\mu} E_{i}=\mathbf{t}^{\left\langle\mu, i^{\prime}\right\rangle} E_{i} T_{\mu}, \quad T_{\mu} F_{i}=\mathbf{t}^{-\left\langle\mu, i^{\prime}\right\rangle} F_{i} T_{\mu}, \\
\Upsilon_{\mu} E_{i}=\mathbf{t}^{\phi\left(\mu, i^{\prime}\right)} E_{i} \Upsilon_{\mu}, \quad \Upsilon_{\mu} F_{i}=\mathbf{t}^{-\phi\left(\mu, i^{\prime}\right)} F_{i} \Upsilon_{\mu},
\end{gathered}
$$

Note that $\dot{\mathbf{U}}[\mathbf{t}]$ is a $\widehat{\mathbf{U}}$-bimodule under the $\mathbf{U}$-module action and under $T_{\mu} \Upsilon_{\nu} 1_{\lambda}=$ $1_{\lambda} T_{\mu} \Upsilon_{\nu}=\mathbf{t}^{\langle\mu, \lambda\rangle+\phi(\nu, \lambda)} 1_{\lambda}$. The enhanced quantum covering group has a useful $\mathbb{Q}(\mathbf{t})$-linear automorphism called a twistor. There are several ways to define such a twistor; we will need the following.

Proposition 6.4. CFLW, Theorems 4.3, 4.12]

(1) There is an automorphism $\mathfrak{X}: \widehat{\mathbf{U}} \rightarrow \widehat{\mathbf{U}}$ defined by

$$
\begin{gathered}
\mathfrak{X}\left(E_{i}\right)=\mathbf{t}_{i}^{2} E_{i} \tilde{T}_{i} \Upsilon_{i}, \quad \mathfrak{X}\left(F_{i}\right)=F_{i} \Upsilon_{-i}, \quad \mathfrak{X}\left(K_{\mu}\right)=T_{-\mu} K_{\mu}, \quad \mathfrak{X}\left(J_{\mu}\right)=T_{2 \mu} J_{\mu}, \\
\mathfrak{X}\left(T_{\mu}\right)=T_{\mu}, \quad \mathfrak{X}\left(\Upsilon_{\mu}\right)=\Upsilon_{\mu}, \quad \mathfrak{X}(v)=\mathbf{t}^{-1} v, \quad \mathfrak{X}(\pi)=-\pi,
\end{gathered}
$$

where if $\mu=\sum_{i \in I} \mu_{i} i, \tilde{T}_{\mu}=\prod_{i \in I} T_{\mu_{i} d_{i} i}$.

(2) There is an automorphism $\mathfrak{X}: \dot{\mathbf{U}}[\mathbf{t}] \rightarrow \dot{\mathbf{U}}[\mathbf{t}]$ such that $\mathfrak{X}\left(u 1_{\lambda} u^{\prime}\right)=\mathfrak{X}(u) 1_{\lambda} \mathfrak{X}\left(u^{\prime}\right)$ for $u, u^{\prime} \in \widehat{\mathbf{U}}$.

Proof. Though we are using a modified version of the twistor defined in [CFLW], essentially the same arguments appearing in loc. cit. work in this case. For completeness, we will provide some arguments for (2) here.

All the relations are clear except for (2.9), (2.10) and (2.11). The Serre relations can be proved as in [CFLW], or alternatively one may use (6.4) below. For the commutator relation,

$$
\begin{aligned}
\mathbf{t}_{i}^{2} E_{i} & \tilde{T}_{i} \Upsilon_{i} F_{j} \Upsilon_{-j}-(-\pi)^{p(i) p(j)} F_{j} \Upsilon_{-j} \mathbf{t}_{i}^{2} E_{i} \tilde{T}_{i} \Upsilon_{i} \\
& =\mathbf{t}^{2 d_{i}-\phi(i, j)+i \cdot j}\left(E_{i} F_{j}-\pi^{p(i) p(j)} \mathbf{t}^{2 p(i) p(j)-i \cdot j+\phi(i, j)-\phi(j, i)} F_{j} E_{i}\right) \tilde{T}_{i} \Upsilon_{i-j} \\
& =\mathbf{t}^{2 d_{i}-\phi(i, j)+i \cdot j}\left(E_{i} F_{j}-\pi^{p(i) p(j)} F_{j} E_{i}\right) \tilde{T}_{i} \Upsilon_{i-j} \\
& =\delta_{i j} \mathbf{t}^{-1} \frac{\tilde{J}_{i} \tilde{K}_{i}-\tilde{K}_{i}^{-1}}{\pi_{i} v_{i}-v_{i}^{-1}} \tilde{T}_{i}=\delta_{i j} \mathbf{t}^{-1} \frac{\tilde{T}_{i}^{2} \tilde{J}_{i} \tilde{T}_{i}^{-1} \tilde{K}_{i}-\tilde{T}_{i} \tilde{K}_{i}^{-1}}{\left(-\pi_{i} \mathbf{t}_{i}^{-1} v_{i}-\mathbf{t}_{i} v_{i}^{-1}\right.} .
\end{aligned}
$$


There is another version of the twistor defined on $\mathbf{f}[\mathbf{t}]=\mathbb{Q}^{\pi}(v, \mathbf{t}) \otimes_{\mathbb{Q}(v)^{\pi}} \mathbf{f}$.

Proposition 6.5. [CFLW, Theorem 2.4] Define a product $*$ on $\mathbf{f}[\mathbf{t}]$ by the following rule: if $x$ and $y$ are homogeneous elements of $\mathbf{f}[\mathbf{t}]$, let $x * y=\mathbf{t}^{\phi\left(|x|,|y|^{\prime}\right)} x y$. Then there is a $\mathbb{Q}(\mathbf{t})$-linear algebra isomorphism $\mathfrak{X}: \mathbf{f} \rightarrow(\mathbf{f}, *)$ defined by

$$
\mathfrak{X}\left(\theta_{i}\right)=\theta_{i}, \quad \mathfrak{X}(v)=\mathbf{t}^{-1} v, \quad \mathfrak{X}(\pi)=-\pi .
$$

Moreover, $\mathfrak{X}$ satisfies $\mathfrak{X}(b)=\mathbf{t}^{\ell(b)} b$ for all $b \in \mathcal{B}(\infty)$, where $\ell(b)$ is some integer depending on $b$.

Now note that the maps $\theta_{i} \mapsto \theta_{i}^{-}=F_{i}$ and $\theta_{i} \mapsto \theta_{i}^{+}=E_{i}$ extend to embeddings $\mathbf{f}[\mathbf{t}] \rightarrow \widehat{\mathbf{U}}^{-}$and $\mathbf{f}[\mathbf{t}] \rightarrow \widehat{\mathbf{U}}^{+}$. These embeddings relate the twistor maps as follows: for $\nu=\sum_{i \in I} \nu_{i} i \in \mathbb{N}[I]$ and $x \in \mathbf{f}[\mathbf{t}]_{\nu}$,

$$
\mathfrak{X}\left(x^{-}\right)=\mathfrak{X}(x)^{-} \Upsilon_{-\nu}, \quad \mathfrak{X}\left(x^{+}\right)=\mathbf{t}^{\bullet(\nu)+\sum 2 d_{i} \nu_{i}} \mathfrak{X}(x)^{+} \tilde{T}_{|x|} \Upsilon_{|x|} .
$$

These equations imply the following result.

Lemma 6.6. Let $b \in \mathcal{B}$. Then $\mathfrak{X}\left(\omega\left(b^{-}\right)\right)=\mathbf{t}^{\ell(b)+\bullet(\nu)} \omega\left(b^{-}\right) \Upsilon_{\nu} \tilde{T}_{-\nu}$.

Proof. Since $\mathfrak{X}\left(x^{+}\right)=\mathbf{t}^{\bullet}(\nu)+\sum 2 d_{i} \nu_{i} \mathfrak{X}(x)^{+} \tilde{T}_{\nu} \Upsilon_{\nu}$ for $x \in \mathbf{f}[\mathbf{t}]_{\nu}$, then in particular for $b \in$ $\mathcal{B}(\nu)_{\nu}$,

$$
\mathfrak{X}\left(\omega\left(b^{+}\right)\right)=\mathfrak{X}\left(b^{+} \pi_{\nu} \tilde{J}_{\nu}\right)=\mathbf{t}^{\ell(b)+\bullet(\nu)+\sum 2 d_{i} \nu_{i}}(-1)^{\sum \nu_{i} d_{i}} \pi_{\nu} \tilde{J}_{\nu} b^{+} \Upsilon_{\nu} \tilde{T}_{-\nu} .
$$

Recall the $\mathbb{Q}(v)^{\pi}$-linear differentials ${ }_{i} r, r_{i}: \mathbf{f} \rightarrow \mathbf{f}$ defined by ${ }_{i} r\left(\theta_{j}\right)=r_{i}\left(\theta_{j}\right)=\delta_{i j}$,

$$
{ }_{i} r(x y)={ }_{i} r(x) y+\pi^{p(x) p(i)} v^{-i \cdot|x|} x{ }_{i} r(y), \quad r_{i}(x y)=\pi^{p(x) p(i)} v^{-i \cdot|x|} r_{i}(x) y+x r_{i}(y) .
$$

These maps trivially extend to $\mathbb{Q}^{\pi}(v, \mathbf{t})$-linear maps on $\mathbf{f}[\mathbf{t}]$.

Lemma 6.7. For any $x \in \mathbf{f}[\mathbf{t}]_{\nu}$,

$$
\mathfrak{X}\left({ }_{i} r(x)\right)=\mathbf{t}^{-\phi\left(i, \nu^{\prime}-i^{\prime}\right)}{ }_{i} r(\mathfrak{X}(x)), \quad \mathfrak{X}\left(r_{i}(x)\right)=\mathbf{t}^{-\phi\left(\nu-i, i^{\prime}\right)} r_{i}(\mathfrak{X}(x)) .
$$

Proof. This is a straightforward verification.

6.2. Twistors on simple modules. Now we will expand on some additional results we shall need regarding the twistors and highest weight modules. Recall from [CHW1] that the Verma module $M(\lambda)$ is the vector space $\mathbf{f}$ with module structure defined as follows: for homogeneous $x \in \mathbf{f}$,

$$
\begin{aligned}
& K_{\mu} 1=v^{\left\langle\mu, \lambda-|x|^{\prime}\right\rangle} x, \quad J_{\mu} x=\pi^{\left\langle\mu, \lambda-|x|^{\prime}\right\rangle} x, \quad F_{i} x=\theta_{i} x, \\
& E_{i} x=\frac{\pi_{i}^{p(x)-p(i)} r_{i}(x)\left(\pi_{i} v_{i}\right)^{\langle i, \lambda\rangle}-v_{i}^{-\left\langle i, \lambda-|x|^{\prime}+i^{\prime}\right\rangle}{ }_{i} r(x)}{\pi_{i} v_{i}-v_{i}^{-1}} .
\end{aligned}
$$

Lemma 6.8. Let $\mathfrak{X}_{\lambda}: M(\lambda)[\mathbf{t}] \rightarrow M(\lambda)[\mathbf{t}]$ be the map defined by

$$
\mathfrak{X}_{\lambda}(x)=\mathbf{t}^{-\phi(\nu, \lambda)} \mathfrak{X}(x)
$$

for homogeneous $x \in \mathbf{f}[\mathbf{t}]_{\nu}$. Then $\mathfrak{X}_{\lambda}(u x)=\mathfrak{X}(u) \mathfrak{X}_{\lambda}(x)$ for all $u \in \widehat{\mathbf{U}}$ and $x \in \mathbf{f}[\mathbf{t}]$.

Proof. It is enough to prove the lemma for the generators of $\widehat{\mathbf{U}}$. First note that $\mathfrak{X}_{\lambda}$ preserves weight spaces, and so the lemma is clear for $T_{\mu}$ and $\Upsilon_{\mu}$; it is also clear that $\mathfrak{X}(v x)=\mathfrak{X}(v) \mathfrak{X}_{\lambda}(x)$ and $\mathfrak{X}(\pi x)=\mathfrak{X}(\pi) \mathfrak{X}_{\lambda}(x)$, whence $\mathfrak{X}_{\lambda}\left(K_{\mu} x\right)=\mathfrak{X}\left(K_{\mu}\right) \mathfrak{X}_{\lambda}(x)$ and $\mathfrak{X}_{\lambda}\left(J_{\mu} x\right)=\mathfrak{X}\left(J_{\mu}\right) \mathfrak{X}_{\lambda}(x)$. It remains to check for $u=E_{i}$ and $u=F_{i}$. 
Let $x \in \mathbf{f}[\mathbf{t}]_{\nu}$. Then for $u=F_{i}$ we have

$$
\mathfrak{X}_{\lambda}\left(F_{i} x\right)=\mathfrak{X}_{\lambda}\left(\theta_{i} x\right)=\mathbf{t}^{\phi\left(i, \nu^{\prime}\right)-\phi(\nu+i, \lambda)} \theta_{i} \mathfrak{X}(x)=F_{i} \Upsilon_{-i} \mathfrak{X}_{\lambda}(x)=\mathfrak{X}\left(F_{i}\right) \mathfrak{X}_{\lambda}(x) .
$$

On the other hand, for $u=E_{i}$ we have

$$
\begin{aligned}
& \mathfrak{X}_{\lambda}\left(E_{i} x\right)=\mathfrak{X}_{\lambda}\left(\frac{\pi_{i}^{p(\nu)-p(i)} r_{i}(x)\left(\pi_{i} v_{i}\right)^{\langle i, \lambda\rangle}-v_{i}^{-\left\langle i, \lambda-\nu^{\prime}+i^{\prime}\right\rangle}{ }_{i} r(x)}{\pi_{i} v_{i}-v_{i}^{-1}}\right) \\
& =\mathbf{t}^{-d_{i}-\phi(\nu-i, \lambda)} \frac{\mathbf{t}_{i}^{2 p(i) p(\nu-i)+\langle i, \lambda\rangle} \pi_{i}^{p(\nu)-p(i)} \mathfrak{X}\left(r_{i}(x)\right)\left(\pi_{i} v_{i}\right)^{\langle i, \lambda\rangle}-\mathbf{t}_{i}^{\left\langle i, \lambda-\nu+i^{\prime}\right\rangle} v_{i}^{-\left\langle i, \lambda-\nu+i^{\prime}\right\rangle} \mathfrak{X}\left({ }_{i} r(x)\right)}{\pi_{i} v_{i}-v_{i}^{-1}} \\
& =\mathbf{t}^{-d_{i}-\phi(\nu-i, \lambda)+d_{i}\left\langle i, \lambda-\nu+i^{\prime}\right\rangle-\phi\left(i, \nu^{\prime}-i^{\prime}\right)} \frac{\mathbf{t}_{i}^{\star} \pi_{i}^{p(\nu)-p(i)} r_{i}(\mathfrak{X}(x))\left(\pi_{i} v_{i}\right)^{\langle i, \lambda\rangle}-v_{i}^{-\left\langle i, \lambda-\nu+i^{\prime}\right\rangle}{ }_{i} r(\mathfrak{X}(x))}{\pi_{i} v_{i}-v_{i}^{-1}}
\end{aligned}
$$

where $\star=2 p(i) p(\nu-i)+d_{i}\langle i, \lambda\rangle-\phi(\nu-i, i)-d_{i}\left\langle i, \lambda-\nu+i^{\prime}\right\rangle+\phi\left(i, \nu^{\prime}-i^{\prime}\right) \equiv_{4} 0$.

Therefore,

$$
\begin{aligned}
\mathfrak{X}_{\lambda}\left(E_{i} x\right) & =\mathbf{t}^{-d_{i}-\phi(\nu-i, \lambda)+d_{i}\left\langle i, \lambda-\nu+i^{\prime}\right\rangle-\phi\left(i, \nu^{\prime}-i^{\prime}\right)} E_{i} \mathfrak{X}(x) \\
& =\mathbf{t}_{i}^{\langle i, \lambda-\nu\rangle} \mathbf{t}^{\phi(i, \lambda-\nu)} \mathbf{t}^{-\phi(\nu, \lambda)+2 d_{i}} E_{i} \mathfrak{X}(x) \\
& =\mathbf{t}_{i}^{2} E_{i} \tilde{T}_{i} \Upsilon_{i} \mathfrak{X}_{\lambda}(x)=\mathfrak{X}\left(E_{i}\right) \mathfrak{X}_{\lambda}(x) .
\end{aligned}
$$

Recall that $V(\lambda)=\mathbf{f} /\left(\theta_{i}^{\langle i, \lambda\rangle+1}: i \in I\right)$ as vector spaces. Then $V(\lambda)[\mathbf{t}]=\mathbf{f}[\mathbf{t}] /\left(\theta_{i}^{\langle i, \lambda\rangle+1}\right.$ : $i \in I)$. Since we further have

$$
\mathfrak{X}_{\lambda}\left(\left(\theta_{i}^{\langle i, \lambda\rangle+1}: i \in I\right)\right)=\left(\theta_{i}^{\langle i, \lambda\rangle+1}: i \in I\right)
$$

we see that $\mathfrak{X}_{\lambda}$ induces a $\mathbb{Q}(\mathbf{t})$-linear isomorphism

$$
\mathfrak{X}_{\lambda}: V(\lambda)[\mathbf{t}] \rightarrow V(\lambda)[\mathbf{t}] .
$$

Lemma 6.9. There is a $\mathbb{Q}(\mathbf{t})$-linear map $\mathfrak{X}_{\lambda}: V(\lambda)[\mathbf{t}] \rightarrow V(\lambda)[\mathbf{t}]$ which satisfies $\mathfrak{X}_{\lambda}\left(\eta_{\lambda}\right)=$ $\eta_{\lambda}$ and $\mathfrak{X}_{\lambda}(u m)=\mathfrak{X}(u) \mathfrak{X}_{\lambda}(m)$ for all $u \in \widehat{\mathbf{U}}$ and $m \in V(\lambda)[\mathbf{t}]$.

6.3. Twistors on tensors. Let $a, a^{\prime}, P\left(\lambda-\lambda^{\prime}, a, a^{\prime}\right)$ be as in $\$ 3.5$. By the results of that section, we have an identification

$$
\dot{\mathbf{U}} 1_{\lambda-\lambda^{\prime}} / P\left(\lambda-\lambda^{\prime}, a, a^{\prime}\right)=N\left(\lambda, \lambda^{\prime}\right)
$$

as $\mathbf{U}$-modules. It is easy to see that $\dot{\mathfrak{X}}$ preserves $\dot{\mathbf{U}} 1_{\lambda-\lambda^{\prime}}$ and $P\left(\lambda-\lambda^{\prime}, a, a^{\prime}\right)$, so in particular $\dot{\mathfrak{X}}: \dot{\mathbf{U}} \rightarrow \dot{\mathbf{U}}$ induces a U-module homomorphism $\mathfrak{X}_{\lambda, \lambda^{\prime}}: N\left(\lambda, \lambda^{\prime}\right) \rightarrow N\left(\lambda, \lambda^{\prime}\right)$ such that

$$
\mathfrak{X}_{\lambda, \lambda^{\prime}}\left(\Delta(u) \eta_{\lambda} \otimes \xi_{-\lambda}\right)=\Delta(\mathfrak{X}(u)) \eta_{\lambda} \otimes \xi_{-\lambda} .
$$

To understand this map better, first we must construct an analogue of Lemma 6.9 for ${ }^{\omega} V(\lambda)[\mathbf{t}]$. To that end, set $\mathfrak{X}^{\prime}=\omega^{-1} \circ \mathfrak{X} \circ \omega$; we note that $\mathfrak{X}^{\prime}\left(E_{i}\right)=\tilde{T}_{-i} \mathfrak{X}\left(E_{i}\right)$, $\mathfrak{X}^{\prime}\left(F_{i}\right)=\mathfrak{X}\left(F_{i}\right) \tilde{T}_{i}, \mathfrak{X}^{\prime}\left(K_{\nu}\right)=\mathfrak{X}\left(K_{\nu}\right), \mathfrak{X}^{\prime}\left(J_{\nu}\right)=\mathfrak{X}\left(J_{\nu}\right), \mathfrak{X}^{\prime}\left(T_{\nu}\right)=\mathfrak{X}\left(T_{\nu}\right)$. We note that in particular,

$$
\begin{gathered}
\Delta\left(\mathfrak{X}\left(E_{i}\right)\right)=\mathfrak{X}\left(E_{i}\right) \otimes \mathfrak{X}^{\prime}\left(\tilde{K}_{i}\right) \Upsilon_{i}+\mathbf{t}_{i}^{2}\left(\Upsilon_{i} \otimes \mathfrak{X}^{\prime}\left(E_{i}\right)\right)\left(\tilde{T}_{i} \otimes \tilde{T}_{i}\right), \\
\Delta\left(\mathfrak{X}\left(F_{i}\right)\right)=\mathfrak{X}\left(F_{i}\right) \otimes \Upsilon_{-i}+\left(\Upsilon_{-i} \mathfrak{X}\left(\tilde{J}_{i} \tilde{K}_{i}\right) \otimes \mathfrak{X}^{\prime}\left(F_{i}\right)\right)\left(\tilde{T}_{-i} \otimes \tilde{T}_{-i}\right) .
\end{gathered}
$$

There is a $\mathbb{Q}(\mathbf{t})$-linear map $\mathfrak{X}_{-\lambda}:{ }^{\omega} V(\lambda)[\mathbf{t}] \rightarrow{ }^{\omega} V(\lambda)[\mathbf{t}]$ which satisfies $\mathfrak{X}_{\lambda}\left(\xi_{-\lambda}\right)=\xi_{-\lambda}$ and $\mathfrak{X}_{-\lambda}(u m)=\mathfrak{X}^{\prime}(u) \mathfrak{X}_{-\lambda}(m)$ for all $u \in \widehat{\mathbf{U}}$ and $m \in{ }^{\omega} V(\lambda)[\mathbf{t}]$; indeed, view $m \in{ }^{\omega} V(\lambda)[\mathbf{t}]$ as an element of $V(\lambda)[\mathbf{t}]$ as an element of $V(\lambda)$ and set $\mathfrak{X}_{-\lambda}(m)=\mathfrak{X}_{\lambda}(m)$. Then

$$
\mathfrak{X}_{-\lambda}(u \cdot m)=\mathfrak{X}_{\lambda}(\omega(u) m)=\mathfrak{X}(\omega(u)) \mathfrak{X}_{\lambda}(m)=\mathfrak{X}^{\prime}(u) \cdot \mathfrak{X}_{-\lambda}(m) .
$$


Then given $w \otimes w^{\prime} \in N\left(\lambda, \lambda^{\prime}\right)$ with $|w|=\zeta$ and $\left|w^{\prime}\right|=\zeta^{\prime}$, we see that

$$
\begin{aligned}
\Delta\left(\mathfrak{X}\left(E_{i}\right)\right)\left(\mathfrak{X}_{\lambda}(w) \otimes \mathfrak{X}_{-\lambda^{\prime}}\left(w^{\prime}\right)=\right. & \mathbf{t}^{\phi\left(i, \zeta^{\prime}\right)} \mathfrak{X}_{\lambda}\left(E_{i} w\right) \otimes \mathfrak{X}_{-\lambda^{\prime}}\left(\tilde{K}_{-i} \cdot w^{\prime}\right) \\
& +\pi^{p(i) p(w)} \mathbf{t}^{2 d_{i}+d_{i}\left\langle i, \zeta+\zeta^{\prime}\right\rangle+\phi(i, \zeta)} \mathfrak{X}_{\lambda}(w) \otimes \mathfrak{X}_{-\lambda^{\prime}}\left(E_{i} \cdot w^{\prime}\right)
\end{aligned}
$$

$$
\begin{aligned}
& \Delta\left(\mathfrak{X}\left(F_{i}\right)\right)\left(\mathfrak{X}_{\lambda}(w) \otimes \mathfrak{X}_{-\lambda^{\prime}}\left(w^{\prime}\right)\right. \\
& \quad=\mathbf{t}^{-\phi\left(i, \zeta^{\prime}\right)} \mathfrak{X}_{\lambda}\left(F_{i} w\right) \otimes \mathfrak{X}_{-\lambda^{\prime}}\left(w^{\prime}\right)+\pi^{p(i) p(w)} \mathbf{t}^{-d_{i}\left\langle i, \zeta-\zeta^{\prime}\right\rangle-\phi(i, \zeta)} \mathfrak{X}_{\lambda}\left(\tilde{J}_{i} \tilde{K}_{i} w\right) \otimes \mathfrak{X}_{-\lambda^{\prime}}\left(F_{i} \cdot w^{\prime}\right)
\end{aligned}
$$

Let $\Lambda_{\lambda}=\left\{\lambda-\nu^{\prime} \mid \nu \in \mathbb{N}[I]\right\}$ and $\mathrm{V}_{\lambda}=\left\{\nu^{\prime}-\lambda \mid \nu \in \mathbb{N}[I]\right\}$. and for $\zeta \in \Lambda_{\lambda}$ (resp. $\left.\zeta \in \mathrm{V}_{\lambda}\right)$ such that $\zeta=\lambda-\nu(\operatorname{resp} . \zeta=\nu-\lambda)$, set $p(\zeta)=p(\nu)$.

Lemma 6.10. There is a function $\varkappa=\varkappa_{\lambda, \lambda^{\prime}}: \Lambda_{\lambda} \times \mathrm{V}_{\lambda^{\prime}} \rightarrow \mathbb{Z}$ satisfying

(1) $\varkappa\left(\zeta-i^{\prime}, \zeta^{\prime}\right) \equiv_{4} \varkappa\left(\zeta, \zeta^{\prime}\right)-\phi\left(i, \zeta^{\prime}\right)$;

(2) $\varkappa\left(\zeta, \zeta^{\prime}+i\right) \equiv_{4} \varkappa\left(\zeta, \zeta^{\prime}\right)+\phi(i, \zeta)+2 d_{i}+\left\langle\tilde{i}, \zeta+\zeta^{\prime}\right\rangle+2 p(\zeta) p(i)$;

(3) $\varkappa\left(\lambda,-\lambda^{\prime}\right) \equiv_{4} 0$.

Proof. Let $\mu, \nu \in \mathbb{N}[I]$ and write $\zeta=\lambda-\nu, \zeta^{\prime}=\mu-\lambda^{\prime}$. Set

$$
\varkappa\left(\lambda,-\lambda^{\prime}\right)=0, \quad \varkappa\left(\lambda-\nu^{\prime},-\lambda^{\prime}\right)=\phi\left(\nu, \lambda^{\prime}\right) .
$$

Then we have defined $\varkappa\left(\lambda-\nu^{\prime}, \mu^{\prime}-\lambda^{\prime}\right)$ for $\operatorname{ht}(\mu)=0$; now assume $\mathrm{ht}(\mu)>0$. Define $\varkappa\left(\lambda-\nu^{\prime}, i^{\prime}+\mu^{\prime}-\lambda^{\prime}\right)=\varkappa\left(\lambda-\nu^{\prime}, \mu^{\prime}-\lambda^{\prime}\right)+\phi\left(i, \lambda-\nu^{\prime}\right)+2 d_{i}+\left\langle\tilde{i}, \lambda-\nu^{\prime}+\mu^{\prime}-\lambda^{\prime}\right\rangle+2 p(\nu) p(i)$.

It is straightforward to check that this definition does not depend on the choice of $i$; that is, if $i+\mu=j+\hat{\mu}$ for some $\hat{\mu} \in \mathbb{N}[I]$, then $\varkappa\left(\lambda-\nu^{\prime}, i^{\prime}+\mu^{\prime}-\lambda^{\prime}\right)=\varkappa\left(\lambda-\nu^{\prime}, j^{\prime}+\hat{\mu}^{\prime}-\lambda^{\prime}\right)$.

Now we need to check that $\varkappa\left(\lambda-\nu^{\prime}, \mu^{\prime}-\lambda\right)$ satisfies (1) and (2). By construction, we see that (1) and (2) hold for ht $(\nu+\mu)=0$, so assume that ht $(\nu+\mu)>0$. By construction, (2) holds and so it suffices to check (1). Write $\mu=\mu_{1}+j$ for some $j \in I$ and $\mu_{1} \in \mathbb{N}[I]$. Then by induction we compute that

$$
\begin{aligned}
& \varkappa\left(\lambda-\nu^{\prime}-i^{\prime}, \mu^{\prime}-\lambda\right) \equiv \equiv_{4} \varkappa\left(\lambda-\nu^{\prime}-i^{\prime}, \mu_{1}^{\prime}-\lambda\right)+\phi\left(j, \lambda-\nu^{\prime}-i^{\prime}\right)+2 d_{j} \\
&+\left\langle\tilde{j}, \lambda-\nu^{\prime}-i^{\prime}+\mu_{1}^{\prime}-\lambda^{\prime}\right\rangle+2 p(\nu+i) p(i) \\
& \equiv_{4} \varkappa\left(\lambda-\nu^{\prime}, \mu_{1}^{\prime}-\lambda\right)-\phi\left(i, \mu_{1}^{\prime}-\lambda^{\prime}\right)+\phi\left(j, \lambda-\nu^{\prime}-i^{\prime}\right)+2 d_{j} \quad+\left\langle\tilde{j}, \lambda-\nu^{\prime}-i^{\prime}+\mu_{1}^{\prime}-\lambda^{\prime}\right\rangle+2 p(\nu+i) p(i) \\
& \equiv_{4} \varkappa\left(\lambda-\nu^{\prime}, \mu_{1}^{\prime}-\lambda\right)+2 p(\nu) p(j)+\phi\left(j, \lambda-\nu^{\prime}\right)+2 d_{j} \quad+\left\langle\tilde{j}, \lambda-\nu^{\prime}+\mu_{1}^{\prime}-\lambda^{\prime}\right\rangle-i \cdot j+2 p(i) p(j)-\phi(j, i)-\phi\left(i, \mu_{1}^{\prime}-\lambda^{\prime}\right) \\
& \equiv_{4} \varkappa\left(\lambda-\nu^{\prime}, \mu^{\prime}-\lambda\right)-i \cdot j+2 p(i) p(j)-\phi(j, i)-\phi\left(i, \mu_{1}^{\prime}-\lambda^{\prime}\right)
\end{aligned}
$$

Now $-i \cdot j+2 p(i) p(j)-\phi(j, i) \equiv_{4}-\phi(i, j)$, and thus we see that

$$
\varkappa\left(\lambda-\nu^{\prime}-i^{\prime}, \mu^{\prime}-\lambda\right) \equiv_{4} \varkappa\left(\lambda-\nu^{\prime}, \mu^{\prime}-\lambda\right)-\phi\left(i, \mu^{\prime}-\lambda^{\prime}\right) .
$$

This finishes the proof.

Proposition 6.11. The $\mathbb{Q}(\mathbf{t})$-linear map $\mathfrak{X}_{\lambda, \lambda^{\prime}}: N\left(\lambda, \lambda^{\prime}\right)[\mathbf{t}] \rightarrow N\left(\lambda, \lambda^{\prime}\right)[\mathbf{t}]$ defined by

$$
\mathfrak{X}_{\lambda, \lambda^{\prime}}\left(w \otimes w^{\prime}\right)=\mathbf{t}^{\varkappa(|v|,|w|)} \mathfrak{X}_{\lambda}(w) \otimes \mathfrak{X}_{-\lambda^{\prime}}\left(w^{\prime}\right),
$$

where $|w|=\lambda-\nu^{\prime}$ and $\left|w^{\prime}\right|=\lambda^{\prime}-\mu^{\prime}$ for $\nu, \mu \in \mathbb{N}[I]$. Then $\mathfrak{X}_{\lambda, \lambda^{\prime}}\left(\Delta(u) w \otimes w^{\prime}\right)=$ $\Delta(\mathfrak{X}(u)) \mathfrak{X}_{\lambda, \lambda^{\prime}}\left(w \otimes w^{\prime}\right)$. 
Proof. Let $w \otimes w^{\prime} \in N\left(\lambda, \lambda^{\prime}\right)$ with $|w|=\zeta$ and $\left|w^{\prime}\right|=\zeta^{\prime}$ for $\nu, \mu \in \mathbb{N}[I]$. It is enough to check when $u$ is a generator. If $u$ is $K_{\nu}, J_{\nu}, T_{\nu}$, or $\Upsilon_{\nu}$ then this is trivial, so it remains to check when $u=E_{i}$ or $u=F_{i}$.

Well, using (6.5), we have

$$
\begin{aligned}
\Delta\left(\mathfrak{X}\left(E_{i}\right)\right) \mathfrak{X}_{\lambda, \lambda^{\prime}}\left(w \otimes w^{\prime}\right)=\mathbf{t}^{\varkappa\left(\zeta, \zeta^{\prime}\right)} \Delta\left(\mathfrak{X}\left(E_{i}\right)\right)\left(\mathfrak{X}_{\lambda}(w) \otimes \mathfrak{X}_{-\lambda^{\prime}}\left(w^{\prime}\right)\right) \\
=\mathbf{t}^{\phi\left(i, \zeta^{\prime}\right)+\varkappa\left(\zeta, \zeta^{\prime}\right)} \mathfrak{X}_{\lambda}\left(E_{i} w\right) \otimes \mathfrak{X}_{-\lambda^{\prime}}\left(\tilde{K}_{-i} \cdot w^{\prime}\right) \\
\quad+\pi^{p(i) p(w)} \mathbf{t}^{\varkappa\left(\zeta, \zeta^{\prime}\right)+2 d_{i}+d_{i}\left\langle i, \zeta+\zeta^{\prime}\right\rangle+\phi(i, \zeta)} \mathfrak{X}_{\lambda}(w) \otimes \mathfrak{X}_{-\lambda^{\prime}}\left(E_{i} \cdot w^{\prime}\right) \\
=\mathbf{t}^{\phi\left(i, \zeta^{\prime}\right)+\varkappa\left(\zeta, \zeta^{\prime}\right)-\varkappa\left(\zeta+i, \zeta^{\prime}\right)} \mathfrak{X}_{\lambda, \lambda^{\prime}}\left(E_{i} w \otimes \tilde{K}_{-i} \cdot w^{\prime}\right) \\
\quad+\pi^{p(i) p(w)} \mathbf{t}^{\varkappa\left(\zeta, \zeta^{\prime}\right)-\varkappa\left(\zeta, \zeta^{\prime}+i\right)+2 d_{i}+d_{i}\left\langle i, \zeta+\zeta^{\prime}\right\rangle+\phi(i, \zeta)} \mathfrak{X}_{\lambda, \lambda^{\prime}}\left(w \otimes E_{i} \cdot w^{\prime}\right) \\
=\mathfrak{X}_{\lambda, \lambda^{\prime}}\left(E_{i} w \otimes \tilde{K}_{-i} \cdot w^{\prime}\right)+\pi^{p(i) p(w)} \mathbf{t}^{2 p(i) p(\zeta)} \mathfrak{X}_{\lambda, \lambda^{\prime}}\left(w \otimes E_{i} \cdot w^{\prime}\right) \\
=\mathfrak{X}_{\lambda, \lambda^{\prime}}\left(\Delta\left(E_{i}\right) w \otimes w^{\prime}\right) .
\end{aligned}
$$

Similarly, using (6.6), we have

$$
\begin{aligned}
& \Delta\left(\mathfrak{X}\left(F_{i}\right)\right) \mathfrak{X}_{\lambda, \lambda^{\prime}}\left(w \otimes w^{\prime}\right)=\mathbf{t}^{\varkappa\left(\zeta, \zeta^{\prime}\right)} \Delta\left(\mathfrak{X}\left(F_{i}\right)\right)\left(\mathfrak{X}_{\lambda}(w) \otimes \mathfrak{X}_{-\lambda^{\prime}}\left(w^{\prime}\right)\right) \\
& =\mathbf{t}^{-\phi\left(i, \zeta^{\prime}\right)+\varkappa\left(\zeta, \zeta^{\prime}\right)} \mathfrak{X}_{\lambda}\left(F_{i} w\right) \otimes \mathfrak{X}_{-\lambda^{\prime}}\left(w^{\prime}\right) \\
& \quad+\pi^{p(i) p(w)} \mathbf{t}^{\varkappa\left(\zeta, \zeta^{\prime}\right)-d_{i}\left\langle i, \zeta+\zeta^{\prime}\right\rangle-\phi(i, \zeta)} \mathfrak{X}_{\lambda}\left(\tilde{J}_{i} \tilde{K}_{i} w\right) \otimes \mathfrak{X}_{-\lambda^{\prime}}\left(F_{i} \cdot w^{\prime}\right) \\
& =\mathbf{t}^{-\phi\left(i, \zeta^{\prime}\right)+\varkappa\left(\zeta, \zeta^{\prime}\right)-\varkappa\left(\zeta-i^{\prime}, \zeta^{\prime}\right)} \mathfrak{X}_{\lambda, \lambda^{\prime}}\left(F_{i} w \otimes w^{\prime}\right) \\
& \quad+\pi^{p(i) p(w)} \mathbf{t}^{\varkappa\left(\zeta, \zeta^{\prime}\right)-\varkappa\left(\zeta, \zeta^{\prime}-i^{\prime}\right)-d_{i}\left\langle i, \zeta+\zeta^{\prime}\right\rangle-\phi(i, \zeta)} \mathfrak{X}_{\lambda, \lambda^{\prime}}\left(\tilde{J}_{i} \tilde{K}_{i} w \otimes F_{i} \cdot w^{\prime}\right) \\
& =\mathfrak{X}_{\lambda, \lambda^{\prime}}\left(\Delta\left(F_{i}\right) w \otimes w^{\prime}\right)
\end{aligned}
$$

Then in particular we have the following result.

Theorem 6.12. Let $b, b^{\prime} \in \mathcal{B}$ and $\lambda, \lambda^{\prime} \in X^{+}$. Set $\zeta=\lambda-\lambda^{\prime}$.

(1) We have $\mathfrak{X}_{\lambda, \lambda^{\prime}}\left(\left(b \diamond b^{\prime}\right)_{\lambda, \lambda^{\prime}}\right)=\mathbf{t}^{f\left(b, b^{\prime}, \zeta\right)}\left(b \diamond b^{\prime}\right)_{\lambda, \lambda^{\prime}}$ for some $f\left(b, b^{\prime}, \zeta\right) \in \mathbb{Z}$.

(2) We have $\mathfrak{X}\left(b \diamond_{\zeta} b^{\prime}\right)=\mathbf{t}^{f\left(b, b^{\prime}, \zeta\right)}\left(b \diamond_{\zeta} b^{\prime}\right)$.

Proof. For (1), first note that the claim $\mathfrak{X}_{\lambda, \lambda^{\prime}}\left(\left(b \diamond b^{\prime}\right)_{\lambda, \lambda^{\prime}}\right)=\mathbf{t}^{f\left(b, b^{\prime}, \lambda, \lambda^{\prime}\right)}\left(b \diamond b^{\prime}\right)_{\lambda, \lambda^{\prime}}$ for some $f\left(b, b^{\prime}, \lambda, \lambda^{\prime}\right) \in \mathbb{Z}$ follows by combining Proposition 6.11. Proposition 6.5 and (6.4). Let $u=b \diamond \zeta b^{\prime}$. Then $\mathfrak{X}\left(u\left(\eta_{\lambda} \otimes \xi_{-\lambda^{\prime}}\right)\right)=\mathbf{t}^{f\left(b, b^{\prime}, \lambda, \lambda^{\prime}\right)}\left(b \diamond b^{\prime}\right)_{\lambda, \lambda^{\prime}} ;$ on the other hand, $\mathfrak{X}\left(u\left(\eta_{\lambda} \otimes\right.\right.$ $\left.\left.\xi_{-\lambda^{\prime}}\right)\right)=\Delta(\mathfrak{X}(u))\left(\eta_{\lambda} \otimes \xi_{-\lambda^{\prime}}\right)$. Therefore, we see that

$$
\Delta(\mathfrak{X}(u))\left(\eta_{\lambda} \otimes \xi_{-\lambda^{\prime}}\right)=\mathbf{t}^{f\left(b, b^{\prime}, \lambda, \lambda^{\prime}\right)}\left(b \diamond b^{\prime}\right)_{\lambda, \lambda^{\prime}}
$$

Let $\lambda^{\prime \prime} \in X^{+}$. Then

$$
\begin{aligned}
\Delta(\mathfrak{X}(u))\left(\eta_{\lambda} \otimes \xi_{-\lambda^{\prime}}\right) & =\Delta(\mathfrak{X}(u)) t\left(\eta_{\lambda+\lambda^{\prime \prime}} \otimes \xi_{-\lambda^{\prime \prime}-\lambda^{\prime}}\right) \\
& =t\left(\Delta(\mathfrak{X}(u)) \eta_{\lambda+\lambda^{\prime \prime}} \otimes \xi_{-\lambda^{\prime \prime}-\lambda^{\prime}}\right) \\
& =t\left(\mathbf{t}^{f\left(b, b^{\prime}, \lambda+\lambda^{\prime \prime}, \lambda^{\prime \prime}+\lambda^{\prime}\right)}\left(b \diamond b^{\prime}\right)_{\lambda+\lambda^{\prime \prime}, \lambda^{\prime \prime}+\lambda^{\prime}}\right) \\
& =\mathbf{t}^{f\left(b, b^{\prime}, \lambda+\lambda^{\prime \prime}, \lambda^{\prime \prime}+\lambda^{\prime}\right)}\left(b \diamond b^{\prime}\right)_{\lambda, \lambda^{\prime}}
\end{aligned}
$$

In particular, we see that $f\left(b, b^{\prime}, \lambda, \lambda^{\prime}\right) \equiv_{4} f\left(b, b^{\prime}, \lambda+\lambda^{\prime \prime}, \lambda^{\prime \prime}+\lambda^{\prime}\right)$, so $\mathbf{t}^{f\left(b, b^{\prime}, \lambda, \lambda^{\prime}\right)}$ is determined by $b, b^{\prime}$, and $\zeta=\lambda-\lambda^{\prime}$, which finishes the proof of (1).

In particular, setting $f\left(b, b^{\prime}, \zeta\right)=f\left(b, b^{\prime}, \lambda, \lambda^{\prime}\right)$,

$$
\Delta(\mathfrak{X}(u))\left(\eta_{\lambda_{0}} \otimes \xi_{-\lambda_{0}^{\prime}}\right)=\mathbf{t}^{f\left(b, b^{\prime}, \zeta\right)}\left(b \diamond b^{\prime}\right)_{\lambda_{0}, \lambda_{0}^{\prime}}
$$


for all $\lambda_{0}, \lambda_{0}^{\prime} \in X^{+}$with $\zeta=\lambda_{0}-\lambda_{0}^{\prime}$, so (2) follows by uniqueness.

\section{REFERENCES}

[BLM] A.A. Beilinson, G. Lusztig, R. McPherson, A geometric setting for the quantum deformation of $\mathrm{GL}_{n}$, Duke Math. J. 61 (1990), 655-677.

[CFLW] S. Clark, Z. Fan, Y. Li and W. Wang, Quantum supergroups III. Twistors, accepted in Comm. Math. Phys., arXiv:1307.7056.

[CHW1] S. Clark, D. Hill and W. Wang, Quantum supergroups I. Foundations, Transform. Groups 18 (2013), 1019-1053.

[CHW2] S. Clark, D. Hill and W. Wang, Quantum supergroups II. Canonical basis, arXiv:1304.7837.

[CHW3] S. Clark, D. Hill and W. Wang, Quantum shuffles and quantum supergroups of basic type, arXiv:1310.7523.

[CW] S. Clark and W. Wang, Canonical basis for quantum osp(1|2), Lett. Math. Phys. 103 (2013), 207-231.

[ETK] H. El Turkey, J. Kujawa, Presenting Schur superalgebras, Pacific Jour. Math. (to appear), arXiv:1209.6327

[EKL] A. Ellis, M. Khovanov and A. Lauda, The odd nilHecke algebra and its diagrammatics, IMRN, 2013, arXiv:1111.1320.

[EL] A. Ellis and A. Lauda, An odd categorification of $U_{q}\left(\mathfrak{s l}_{2}\right)$, arXiv:1307.7816v2

[FL] Z. Fan and Y. Li, A geometric setting for quantum osp(1|2), Trans. Amer. Math. Soc. (to appear), arXiv:1305.0710.

[KKO] S.-J. Kang, M. Kashiwara and S.-J. Oh, Supercategorification of quantum Kac-Moody algebras II, arXiv:1303.1916.

[KKT] S.-J. Kang, M. Kashiwara and S. Tsuchioka, Quiver Hecke superalgebras, arXiv:1107.1039.

[HW] D. Hill and W. Wang, Categorification of quantum Kac-Moody superalgebras, Trans. Amer. Math. Soc. (to appear), arXiv:1202.2769v2.

[K91] M. Kashiwara, On crystal bases of the Q-analogue of universal enveloping algebras, Duke Math. J. 63 (1991), 456-516.

[K93] M. Kashiwara, Global crystal bases of quantum groups, Duke Math. J. 69(2), 455-485, 1993.

[KL] M. Khovanov and A. Lauda, A diagrammatic approach to categorification of quantum groups III, Quantum Topology 1 (2010), 1-92.

[L90] G. Lusztig, Canonical bases arising from quantized enveloping algebras, J. Amer. Math. Soc. 3 (1990), 447-498.

[L92] G. Lusztig, Canonical bases in tensor products, Proc. Nat. Acad. Sci. U.S.A. 89 (1992), no. 17, 8177-8179.

[L93] G. Lusztig, Introduction to Quantum Groups, Progress in Math. 110, Birkhäuser 1993.

[MW] V. Mikhaylov and E. Witten, Branes and supergroups, to appear.

[W] W. Wang, Double affine Hecke algebras for the spin symmetric group, Math. Res. Lett. 16 (2009), 1071-1085.

[Web13] B. Webster, Knot invariants and higher representation theory I, arXiv:1309.3796.

Department of Mathematics, University of Virginia, Charlottesville, VA 22904

E-mail address: sic5ag@virginia.edu 